\title{
UNBOUNDED DERIVATIONS, FREE DILATIONS, AND INDECOMPOSABILITY RESULTS FOR II FACTORS $_{1}$
}

\author{
YOANN DABROWSKI AND ADRIAN IOANA
}

\begin{abstract}
We give sufficient conditions, in terms of the existence of unbounded derivations satisfying certain regularity properties, which ensure that a $\mathrm{II}_{1}$ factor $M$ is prime or has at most one Cartan subalgebra. For instance, we prove that if there exists a real closable unbounded densely defined derivation $\delta: M \rightarrow L^{2}(M) \bar{\otimes} L^{2}(M)$ whose domain contains a non-amenability set, then $M$ is prime. If $\delta$ is moreover "algebraic" (i.e. its domain $M_{0}$ is finitely generated, $\delta\left(M_{0}\right) \subset M_{0} \otimes M_{0}$ and $\left.\delta^{*}(1 \otimes 1) \in M_{0}\right)$, then we show that $M$ has no Cartan subalgebra. We also give several applications to examples from free probability. Finally, we provide a class of countable groups $\Gamma$, defined through the existence of an unbounded cocycle $b: \Gamma \rightarrow \mathbb{C}(\Gamma / \Lambda)$, for certain subgroups $\Lambda<\Gamma$, such that the $\mathrm{II}_{1}$ factor $L^{\infty}(X) \rtimes \Gamma$ has a unique Cartan subalgebra, up to unitary conjugacy, for any free ergodic probability measure preserving (pmp) action $\Gamma \curvearrowright(X, \mu)$.
\end{abstract}

\section{INTRODUCTION AND STATEMENT OF MAIN RESULTS}

1.1. Background. A central theme in the theory of von Neumann algebras is to investigate various decompositions of $\mathrm{II}_{1}$ factors, such as tensor product and Cartan decompositions. Recall that a $\mathrm{II}_{1}$ factor $M$ is prime if it cannot be written as the tensor product $M=M_{1} \bar{\otimes} M_{2}$ of two $\mathrm{II}_{1}$ factors. Also, a maximal abelian von Neumann subalgebra $A \subset M$ is a Cartan subalgebra if its normalizer, $\mathcal{N}_{M}(A)=$ $\left\{u \in \mathcal{U}(M) \mid u A u^{*}=A\right\}$, generates a weakly dense subalgebra of $M$.

The general goal of this paper is to provide new classes of $\mathrm{II}_{1}$ factors that are prime and have at most one Cartan subalgebra. We start by giving a short history of results of this type.

In Po83, Popa proved that uncountable free groups give rise to $\mathrm{II}_{1}$ factors that are prime and do not have Cartan subalgebras. The first examples of separable such $\mathrm{II}_{1}$ factors were obtained in the mid $90 \mathrm{~s}$ as an application of free probability theory. Thus, Voiculescu showed that any $\mathrm{II}_{1}$ factor admitting a generating set whose free entropy dimension is greater than 1 has no Cartan subalgebra Vo96. In particular, the free group factors, $L\left(\mathbb{F}_{n}\right)$, with $2 \leqslant n \leqslant \infty$, do not have Cartan subalgebras. Subsequently, Ge proved that the free group factors are also prime Ge98.

During the last decade, Popa's deformation/rigidity theory has generated spectacular progress in the study of $\mathrm{II}_{1}$ factors. In particular, it has led to the first

Received by the editors May 4, 2013 and, in revised form, May 4, 2014.

2010 Mathematics Subject Classification. Primary 46L36; Secondary 28D15, 37A20.

The first author was partially supported by ANR grant NEUMANN.

The second author was partially supported by NSF Grant DMS \#1161047, NSF Career Grant DMS \#1253402, and a Sloan Foundation Fellowship. 
classes of $\mathrm{II}_{1}$ factors that have a unique Cartan subalgebra, up to unitary conjugacy. We highlight here three major advances in this direction and refer the reader to the surveys Po07b, Va10, Io12 for more information. In Po06a] Popa showed that any $\mathrm{II}_{1}$ factor has at most one Cartan subalgebra which satisfies a certain combination of deformation and rigidity properties. Later on, Ozawa and Popa found the first class of $\mathrm{II}_{1}$ factors that have a unique arbitrary Cartan subalgebra OP10. More precisely, they showed that any $\mathrm{II}_{1}$ factor $L^{\infty}(X) \rtimes \mathbb{F}_{n}$ arising from a free ergodic profinite probability measure preserving (pmp) action $\mathbb{F}_{n} \curvearrowright(X, \mu)$ of a free group $\mathbb{F}_{n}(2 \leqslant n \leqslant \infty)$ has a unique Cartan subalgebra, up to unitary conjugacy. Very recently, Popa and Vaes vastly generalized this result by proving that it holds for any free ergodic pmp action $\mathbb{F}_{n} \curvearrowright(X, \mu)$ PV14a].

In the last ten years, the primeness and absence of Cartan subalgebras of the free group factors Vo96, Ge98, have been generalized and strengthened in many ways. Firstly, Ozawa proved that $\mathrm{II}_{1}$ factors arising from hyperbolic groups $\Gamma$ are solid: the relative commutant $A^{\prime} \cap L(\Gamma)$ of any diffuse subalgebra $A \subset L(\Gamma)$ is amenable Oz04. In particular, $L(\Gamma)$ and all of its non-amenable subfactors are prime. Secondly, using a technique based on closable derivations, Peterson was able to show that $\mathrm{II}_{1}$ factors arising from groups with positive first $\ell^{2}$-Betti number are prime $\mathrm{Pe} 09 \mathrm{~b}$.

Using his deformation/rigidity theory, Popa then found a new proof of solidity for $L\left(\mathbb{F}_{n}\right)$ Po07a]. Popa's approach relies on the remarkable discovery Po08] that the presence of a spectral gap can be viewed as a source of rigidity. This spectral gap rigidity principle has since been the catalyst behind many developments in deformation/rigidity theory. Thus, it was a crucial ingredient in the finding of $\mathrm{II}_{1}$ factors with a unique Cartan subalgebra OP10, PV14a. In OP10, Ozawa and Popa used the spectral gap rigidity principle to show that the free group factors enjoy a structural property, called strong solidity, which strengthens both solidity and absence of Cartan subalgebras: the normalizer of any diffuse subalgebra $A \subset$ $L\left(\mathbb{F}_{n}\right)$ is amenable. Also using Popa's spectral gap rigidity principle, Chifan and Sinclair showed that, more generally, the group von Neumann algebra of any icc hyperbolic group is strongly solid [CS13].

1.2. Statement of main results. In this paper, we use Popa's deformation/ rigidity theory to prove primeness and absence/uniqueness of Cartan subalgebras for $\mathrm{II}_{1}$ factors in the presence of unbounded closable derivations satisfying certain regularity properties.

Our first result shows that if a non-amenable $\mathrm{II}_{1}$ factor $M$ admits a closable unbounded derivation into its coarse bimodule which has a "large" domain, then $M$ is prime. To make this precise, let us introduce a definition. If $M$ is a $\mathrm{II}_{1}$ factor, then we say that a finite set $S \subset M$ is a non-amenability set for $M$ if there exists a constant $K>0$ such that $\|\xi\|_{2} \leqslant K \sum_{x \in S}\|x \xi-\xi x\|_{2}$, for every vector $\xi \in L^{2}(M) \bar{\otimes} L^{2}(M)$. Note that by Connes' theorem [Co76], $M$ is non-amenable if and only if it admits a non-amenability set.

Theorem 1.1. Let $M$ be a non-amenable $I_{1}$ factor and $M_{0}$ be a weakly dense *-subalgebra which contains a non-amenability set for $M$. Assume that there exists a real closable unbounded derivation $\delta: M_{0} \rightarrow\left(L^{2}(M) \bar{\otimes} L^{2}(M)\right)^{\oplus \infty}$.

Then $M$ is not $L^{2}$-rigid. In particular, $M$ is prime and does not have property Gamma. 
Recall that if $\mathcal{H}$ is an $M-M$ bimodule, then a linear map $\delta: M_{0} \rightarrow \mathcal{H}$ is a derivation if it verifies $\delta(x y)=x \delta(y)+\delta(x) y$, for all $x, y \in M_{0}$. We say that $\delta$ is bounded if $\sup _{x \in M_{0},\|x\| \leqslant 1}\|\delta(x)\|<\infty$. Note that $\delta$ is bounded if and only if it is inner; i.e. there exists $\xi \in \mathcal{H}$ such that $\delta(x)=x \xi-\xi x$, for all $x \in M_{0}$ (see the proof of [Pe09a, Theorem 2.2]). Also, recall that a $\mathrm{II}_{1}$ factor $M$ has property Gamma of Murray and von Neumann MvN43] if there exists a sequence $u_{n} \in \mathcal{U}(M)$ such that $\tau\left(u_{n}\right)=0$, for all $n$, and $\left\|u_{n} x-x u_{n}\right\|_{2} \rightarrow 0$, for all $x \in M$.

A $\mathrm{II}_{1}$ factor $M$ is $L^{2}$-rigid in the sense of Peterson [Pe09b, Definition 4.1] if any completely positive semigroup $\phi_{t}=\exp \left(-t \delta^{*} \bar{\delta}\right)$ arising from a real closable densely defined derivation $\delta$ into a multiple of the coarse bimodule converges uniformly to $\mathrm{id}_{M}$ on the unit ball of $M$, as $t \rightarrow 0$. By $\mathrm{Pe} 09 \mathrm{~b}$, Theorem 1.1], if a $\mathrm{II}_{1}$ factor is not prime or has property Gamma, then it is $L^{2}$-rigid. On the other hand, if an icc group $\Gamma$ admits an unbounded cocycle $b: \Gamma \rightarrow \ell^{2}(\Gamma)^{\oplus \infty}$, then $L(\Gamma)$ is not $L^{2}$-rigid [Pe09b, Example 2.2]. Theorem 1.1 generalizes this fact and provides new examples of non- $L^{2}$-rigid factors.

Note that if $M_{0}$ does not contain a non-amenability set for $M$, then Theorem 1.1 fails in general (see Remark 4.2).

By Vo96, Ge98, $\mathrm{II}_{1}$ factors which admit a set of generators whose microstates free entropy is finite, $\chi\left(X_{1}, \ldots, X_{n}\right)>-\infty$, do not have property Gamma, are prime and do not have Cartan subalgebras. In [Vo98b] Voiculescu introduced a non-microstates free entropy $\chi^{*}\left(X_{1}, \ldots, X_{n}\right)$. The two entropies satisfy $\chi \leqslant \chi^{*}$ by [BCG03] and are believed to be equal whenever Connes' embedding conjecture holds. Nevertheless, unlike its microstates counterpart, the non-microstates free entropy has not yet found applications to von Neumann algebras. In particular, it is an open problem whether the above indecomposability results hold under the assumption $\chi^{*}\left(X_{1}, \ldots, X_{n}\right)>-\infty$. In this direction, it was shown in Da10a that if the assumption that $\chi^{*}\left(X_{1}, \ldots, X_{n}\right)>-\infty$ is replaced with the stronger assumption that the free Fisher information is finite $\Phi^{*}\left(X_{1}, \ldots, X_{n}\right)<\infty$, then the von Neumann algebra $M$ generated by $\left\{X_{1}, \ldots, X_{n}\right\}$ is a $\mathrm{II}_{1}$ factor without property Gamma.

In this paper, we show that if we further strengthen the condition $\Phi^{*}\left(X_{1}, \ldots, X_{n}\right)$ $<\infty$, then we can conclude that $M$ is prime and does not have a Cartan subalgebra. Firstly, as a consequence of Theorem 1.1 we deduce the following.

Corollary 1.2. Let $(M, \tau)$ be a tracial von Neumann algebra which is generated by $n \geqslant 2$ algebraically free self-adjoint elements $X_{1}, \ldots, X_{n}$. Assume that either

- $\mathcal{J}_{p}\left(X_{i}: \mathbb{C}\left\langle X_{1}, \ldots, X_{i-1}, X_{i+1}, \ldots, X_{n}\right\rangle\right)$ exists and belongs to $M$, for all $p \in\{1,2\}$ and every $i \in\{1, \ldots, n\}$, or

- $\Phi^{*}\left(X_{1}, \ldots, X_{n}\right)<\infty$ and $n \geqslant 3$.

Then $M$ is a non- $L^{2}$-rigid $I I_{1}$ factor. In particular, $M$ is prime and does not have property Gamma.

To recall the definition of the $p$-th order conjugate variable

$$
\xi_{p, i}=\mathcal{J}_{p}\left(X_{i}: \mathbb{C}\left\langle X_{1}, \ldots, X_{i-1}, X_{i+1}, \ldots, X_{n}\right\rangle\right),
$$

for $p \in\{1,2\}$, let $M_{0}$ be the $*$-algebra generated by $X_{1}, \ldots, X_{n}$. Let $\delta_{i}: M_{0} \rightarrow$ $L^{2}(M) \bar{\otimes} L^{2}(M)$ be the partial free difference quotient derivation given by $\delta_{i}\left(X_{j}\right)=$ $\delta_{i, j} 1 \otimes 1$. Then the first and second order conjugate variables are defined as $\xi_{1, i}=$ $\delta_{i}^{*}(1 \otimes 1) \in L^{2}(M)$ and $\xi_{2, i}=\delta_{i}^{*}\left(\xi_{1, i} \otimes 1\right) \in L^{2}(M)$ whenever these formulas make 
sense (see Vo98b, Definition 3.1]). If the first order conjugate variables $\xi_{1, i}$ exist, then the free Fisher information is given by $\Phi^{*}\left(X_{1}, \ldots, X_{n}\right)=\sum_{i=1}^{n}\left\|\xi_{1, i}\right\|_{2}^{2}$.

Secondly, by assuming a Lipschitz condition on conjugate variables [Da14, Definition 1] we are able to deduce the absence of Cartan subalgebras.

Theorem 1.3. Let $(M, \tau)$ be a tracial von Neumann algebra which is generated by $n \geqslant 2$ algebraically free self-adjoint elements $X_{1}, \ldots, X_{n}$. Let $M_{0}$ be the *-algebra generated by $X_{1}, \ldots, X_{n}$. For $1 \leqslant i \leqslant n$, denote by $\delta_{i}: M_{0} \rightarrow L^{2}(M) \bar{\otimes} L^{2}(M)$ the free difference quotient $\delta_{i}\left(X_{j}\right)=\delta_{i, j} 1 \otimes 1$.

Let $\delta=\left(\delta_{1}, \ldots, \delta_{n}\right): M_{0} \rightarrow\left(L^{2}(M) \bar{\otimes} L^{2}(M)\right)^{\oplus_{n}}$ and $\bar{\delta}$ be the closure of $\delta$. Assume that $1 \otimes 1$ is in the domain of $\delta_{i}^{*}$ and denote $\xi_{i}=\delta_{i}^{*}(1 \otimes 1)$. Moreover, assume that $\xi_{i}$ is in the domain of $\bar{\delta}$ and $\bar{\delta}\left(\xi_{i}\right) \in\left(M \bar{\otimes} M^{o p}\right)^{\oplus n}$, for all $1 \leqslant i \leqslant$ $n$. Here, $M^{o p}$ denotes the opposite algebra of $M$, and we consider the inclusion $M \bar{\otimes} M^{o p} \subset L^{2}\left(M \bar{\otimes} M^{o p}\right) \cong L^{2}(M) \bar{\otimes} L^{2}(M)$.

Then $M$ is a $I I_{1}$ factor and does not have a Cartan subalgebra. Moreover, $M \bar{\otimes} Q$ does not have a Cartan subalgebra for any $I I_{1}$ factor $Q$.

In the second part of this paper we establish absence (or uniqueness) of Cartan subalgebras for $\mathrm{II}_{1}$ factors $M$ admitting certain unbounded "algebraic" derivations.

Firstly, we show that, under fairly general conditions, the existence of a finitely generated weakly dense *-subalgebra $M_{0} \subset M$, a von Neumann subalgebra $B \subset$ $M$, and an unbounded derivation $\delta: M_{0} \rightarrow L^{2}\left(\left\langle M, e_{B}\right\rangle\right)$ such that $\delta\left(M_{0}\right) \subset$ span $\left(M_{0} e_{B} M_{0}\right)$ implies that $M$ has no Cartan subalgebras. For a precise statement, see Theorem 7.1 .

Let us state two corollaries of this result. By Theorem 1.1 if a $\mathrm{II}_{1}$ factor $M$ admits a closable unbounded derivation $\delta: M_{0} \rightarrow L^{2}(M) \bar{\otimes} L^{2}(M)$ whose domain contains a non-amenability set, then it is prime. We believe that the existence of such a derivation $\delta$ should also imply that $M$ does not have a Cartan subalgebra. However, proving this seems out of reach with the methods that are currently available. Nevertheless, as a consequence of Theorem 7.1 we are able to confirm this conjecture if $\delta$ is algebraic.

Corollary 1.4. Let $M$ be a non-amenable $I I_{1}$ factor and $M_{0} \subset M$ be a finitely generated weakly dense *-subalgebra which contains a non-amenability set for $M$. Assume that there exists a real unbounded derivation $\delta: M_{0} \rightarrow L^{2}(M) \bar{\otimes} L^{2}(M)$ such that $\delta\left(M_{0}\right) \subset M_{0} \otimes M_{0}, 1 \otimes 1$ is in the domain of $\delta^{*}$ and $\delta^{*}(1 \otimes 1) \in M_{0}$.

Then $M$ does not have a Cartan subalgebra.

Corollary 1.4 generalizes part of [Io15. Corollary 1.5]. Indeed, it implies that the free product $M=M_{1} * M_{2}$ of any two finitely generated $\mathrm{II}_{1}$ factors does not have a Cartan subalgebra. However, we are unaware of any example of a $\mathrm{II}_{1}$ factor which satisfies the hypothesis of Corollary 1.4 and is not essentially a free product (see also Remark 3.5.).

Remark 1.5. As we explain below, Corollary 1.2. Theorem 1.3 and Corollary 1.4 generalize a number of results in the literature that were originally obtained by using free probability theory, under the additional assumption that the algebras $M$ in question are embeddable into $R^{\omega}$. Our contribution is to prove these results without requiring that $M$ embeds into $R^{\omega}$. Note that Connes's embedding problem of deciding whether every separable tracial von Neumann algebra embeds into $R^{\omega}$ remains a major open problem. 
Firstly, Corollary 1.2 implies that if $X_{1}, \ldots, X_{n}$ are self-adjoint elements of a tracial von Neumann algebra $(M, \tau)$, for some $n \geqslant 2$, and $S_{1}, \ldots, S_{n} \in M$ are free semicircular elements which are free from $X_{1}, \ldots, X_{n}$, then $X_{1}^{\varepsilon}=X_{1}+\varepsilon S_{1}, \ldots, X_{n}^{\varepsilon}=$ $X_{n}+\varepsilon S_{n}$ generate a prime $\mathrm{II}_{1}$ factor, for any $\varepsilon>0$. Indeed, by Vo98b, Corollary 3.9] $X_{1}^{\varepsilon}, \ldots, X_{n}^{\varepsilon}$ satisfy the above assumption on conjugate variables. Moreover, by using [Io15, we can show that the $\mathrm{II}_{1}$ factor generated by $X_{1}^{\varepsilon}, \ldots, X_{n}^{\varepsilon}$ does not have a Cartan subalgebra (see Theorem6.1). In the case the von Neumann algebra generated by $\left\{X_{1}, \ldots, X_{n}\right\}$ is embeddable into $R^{\omega}$, the last two facts follow from [Vo96, Vo98a, Ge98. Secondly, Theorem 1.3 generalizes a result of [Da14] where by using microstates free entropy techniques and [Vo96, Sh09] it was shown that if $M$ is embeddable into $R^{\omega}$, then it has no Cartan subalgebras. Finally, note that if $M$ is embeddable into $R^{\omega}$, then Corollary 1.4 is a consequence of Sh09.

As a consequence of Theorem 7.1 we also provide a general criterion for the absence of Cartan subalgebras in amalgamated free product $\mathrm{II}_{1}$ factors.

Corollary 1.6. Let $\left(M_{1}, \tau_{1}\right)$ and $\left(M_{2}, \tau_{2}\right)$ be tracial von Neumann algebras with a common von Neumann subalgebra such that $\tau_{1 \mid B}=\tau_{2 \mid B}$ and denote $M=M_{1} *_{B} M_{2}$. Assume that there exist unitary elements $u \in M_{1}$ and $v, w \in M_{2}$ such that $E_{B}(u)=$ $E_{B}(v)=E_{B}(w)=E_{B}\left(w^{*} v\right)=0$. Suppose that either $u B u^{*} \perp B$ or $v B v^{*} \perp B$.

Then $M$ is a $I I_{1}$ factor, does not have a Cartan subalgebra and does not have property Gamma.

For the definition of the amalgamated free product of tracial von Neumann algebras, see [Po93] and [VDN92]. Following [Po83], we say that two von Neumann subalgebras $B_{1}, B_{2}$ of a tracial von Neumann algebra $(M, \tau)$ are orthogonal if $\tau\left(b_{1} b_{2}\right)=\tau\left(b_{1}\right) \tau\left(b_{2}\right)$, for all $b_{1} \in B_{1}$ and $b_{2} \in B_{2}$.

Finally, we provide a new class of $\mathrm{II}_{1}$ factors that have a unique Cartan subalgebra, up to unitary conjugacy. By [PV14a, Definition 1.4] a countable group $\Gamma$, whose every free ergodic pmp action $\Gamma \curvearrowright(X, \mu)$ gives rise to a $\mathrm{II}_{1}$ factor with a unique Cartan subalgebra, is called $\mathcal{C}$-rigid (Cartan rigid). In a recent breakthrough, Popa and Vaes proved that all weakly amenable groups with a positive first $\ell^{2}$-Betti number and all non-elementary hyperbolic groups are $\mathcal{C}$-rigid PV14a, PV14b. Most recently, it was shown in [Io15, Theorem 1.1] that any amalgamated free product group $\Gamma=\Gamma_{1} *_{\Lambda} \Gamma_{2}$ such that $\left[\Gamma_{1}: \Lambda\right] \geqslant 2,\left[\Gamma_{2}: \Lambda\right] \geqslant 3$, and $\bigcap_{i=1}^{m} g_{i} \Lambda g_{i}^{-1}$ is finite, for some $g_{1}, \ldots, g_{m} \in \Gamma$, is $\mathcal{C}$-rigid.

Our next result gives a cohomological criterion, in terms of the existence of a certain unbounded algebraic cocycle, for a group $\Gamma$ to be $\mathcal{C}$-rigid. Recall that if $V$ is a left $\Gamma$-module, then a map $b: \Gamma \rightarrow V$ is said to be a cocycle if it satisfies the relation $b(g h)=b(g)+g \cdot b(h)$, for all $g, h \in \Gamma$.

Theorem 1.7. Let $\Gamma$ be a countable icc group, $\Lambda<\Gamma$ be a subgroup and assume that there exists an unbounded cocycle $b: \Gamma \rightarrow \mathbb{C}(\Gamma / \Lambda)$ satisfying $b_{\mid \Lambda} \equiv 0$. Additionally, assume that

- $\bigcap_{i=1}^{m} g_{i} \Lambda g_{i}^{-1}$ is finite, for some $g_{1}, g_{2}, \ldots, g_{m} \in \Gamma$,

- there exists an increasing sequence $\left\{\Gamma_{n}\right\}_{n} \geqslant 1$ of finitely generated subgroups of $\Gamma$ such that $\bigcup_{n \geqslant 1} \Gamma_{n}=\Gamma$ and $b\left(\Gamma_{n}\right) \subset \mathbb{C}\left(\Gamma_{n} \Lambda / \Lambda\right)$, for all $n \geqslant 1$,

- $L(\Gamma)$ does not have property Gamma and $\Lambda$ is not co-amenable in $\Gamma$.

Then $L^{\infty}(X)$ is the unique Cartan subalgebra of $L^{\infty}(X) \rtimes \Gamma$, up to unitary conjugacy, for any free ergodic pmp action $\Gamma \curvearrowright(X, \mu)$. 
Since amalgamated free product groups admit such algebraic cocycles, this theorem generalizes Io15, Theorem 1.1] recalled above. Moreover, it leads to new examples of $\mathcal{C}$-rigid groups.

Corollary 1.8. Let $G$ be a countable group, $\Lambda<G$ be a subgroup, and $\theta: \Lambda \rightarrow G$ be an injective group homomorphism such that $\Lambda \neq G$ and $\theta(\Lambda) \neq G$. Denote by $\Gamma=H N N(G, \Lambda, \theta)$ the corresponding $H N N$ extension. Assume that $\bigcap_{i=1}^{m} g_{i} \Lambda g_{i}^{-1}$ is finite, for some $g_{1}, g_{2}, \ldots, g_{m} \in \Gamma$.

Then $L^{\infty}(X)$ is the unique Cartan subalgebra of $L^{\infty}(X) \rtimes \Gamma$, up to unitary conjugacy, for any free ergodic pmp action $\Gamma \curvearrowright(X, \mu)$.

Corollary 1.8 strengthens and generalizes the main result of [FV12]. Indeed, [FV12, Theorem 1.1] shows that if we moreover assume that $G$ contains a nonamenable subgroup with the relative property $(\mathrm{T})$ or two commuting non-amenable subgroups and that $\Lambda$ is amenable, then $L^{\infty}(X) \rtimes \Gamma$ has a unique group measure space Cartan subalgebra, for any free ergodic pmp action $\Gamma \curvearrowright(X, \mu)$.

1.3. Comments on the proofs. Let us say a few words about the proofs of Theorems 1.1. Theorem 1.3 and Corollary 1.4. since they are representative of the proofs of all the results stated above. Consider a real unbounded closable derivation $\delta: M_{0} \rightarrow\left(L^{2}(M) \bar{\otimes} L^{2}(M)\right)^{\oplus n}$, for some $1 \leqslant n \leqslant \infty$, which satisfies the corresponding regularity conditions. Recall that $M_{0}$ contains a non-amenability set $S$ for $M$. Our goal is to prove either that $M$ is not $L^{2}$-rigid (as in Theorem 1.1) or that $M$ has no Cartan subalgebra (as in Theorem 1.3 and Corollary 1.4).

To this end, we use results from free probability theory to construct a malleable deformation of $M$, in the sense of Popa. This consists of a tracial von Neumann algebra $\tilde{M}$ containing $M$ and a pointwise $\|.\|_{2}$-continuous path $\left\{\alpha_{t}\right\}_{t \geqslant 0}$ of $*-$ homomorphisms $\alpha_{t}: M \rightarrow \tilde{M}$ such that $\alpha_{0}=\operatorname{id}_{M}$. Moreover, the pair $\left(\tilde{M},\left\{\alpha_{t}\right\}_{t \geqslant 0}\right)$ is a "dilation of $\delta$ " in the following broad sense: the $\operatorname{limit}_{t \rightarrow 0} \frac{1}{t}\left\|\alpha_{t}(x)-x\right\|_{2}$ exists and is determined by $\delta$, for all $x \in M_{0}$.

By [Da10b, any real closable derivation admits a dilation. Moreover, for derivations into a multiple of the coarse bimodule, such as $\delta$, Da10b provides additional information on the dilation. More precisely, if $M_{t}$ denotes the von Neumann algebra generated by $M$ and $\alpha_{t}(M)$, then the $M$ - $M$ bimodule $L^{2}\left(M_{t}\right) \ominus L^{2}(M)$ is contained in a multiple of the coarse bimodule, for any $t \geqslant 0$.

If $M$ is $L^{2}$-rigid, then the semigroup $\phi_{t}=\exp \left(-t \delta^{*} \bar{\delta}\right)$ converges uniformly to $\mathrm{id}_{M}$ on the unit ball of $M$, as $t \rightarrow 0$. This readily entails that $\alpha_{t}$ converges uniformly to $\operatorname{id}_{M}$, as $t \rightarrow 0$. As a consequence, for every small enough $t$, there exists a unitary $u_{t} \in \tilde{M}$ such that $u_{t} \alpha_{t}(M) u_{t}^{*} \subset M$. Since $S \subset M$ is a non-amenability set, a variation of Popa's spectral gap argument Po08] (see Lemma 2.7]) allows us to find a constant $K>0$ such that

$(*) \quad\left\|\alpha_{t}(x)-E_{M}\left(\alpha_{t}(x)\right)\right\|_{2} \leqslant K \sum_{y \in S}\left\|\alpha_{t}(y)-y\right\|_{2}$,

for all $x \in(M)_{1}$ and every $t \geqslant 0$.

Since $S \subset M_{0}$ and $\alpha_{t}$ dilates $\delta$, this inequality implies that $\delta$ is bounded. This is a contradiction, proving that $M$ is not $L^{2}$-rigid, as claimed by Theorem 1.1.

Now, assume that $\delta$ is the free difference quotient and the conjugate variables satisfy a Lipschitz condition (as in Theorem 1.3) or $\delta$ is algebraic (as in Corollary 
1.4). Then results from Da14 and Sh09 imply that the dilation algebra $\tilde{M}$ can be taken equal to $M * L\left(\mathbb{F}_{\infty}\right)$. In this case, we say that $\delta$ admits a "free dilation".

We then use techniques from Popa's deformation/rigidity theory to prove that $M$ does not have a Cartan subalgebra. In particular, we employ the recent work [Io15] (which notably uses [PV14a]) on the structure of normalizers of subalgebras of amalgamated free product algebras. By combining Io15 with Lemma 2.7 we show that if $M$ has a Cartan subalgebra, then $(*)$ holds. As above, this provides a contradiction.

1.4. Organization of the paper. Besides the introduction, this paper has seven other sections. In Section 2 we record several notions and results that we will later use. In Section 3 we recall known results on dilating derivations. Sections 4-8 are devoted to the proofs of our main results.

\section{Preliminaries}

2.1. Terminology. We work with tracial von Neumann algebras $(M, \tau)$, i.e. von Neumann algebras $M$ endowed with a faithful, normal, tracial state $\tau$. We denote by $\|x\|_{2}=\tau\left(x^{*} x\right)^{1 / 2}$ the 2 -norm associated to $\tau$ and by $\|x\|$ the operator norm. We denote by $\mathcal{Z}(M)$ the center of $M$, by $\mathcal{U}(M)$ the group of unitaries of $M$ and by $(M)_{1}=\{x \in M \mid\|x\| \leqslant 1\}$ the unit ball of $M$. We always assume that $M$ is separable, unless it is a subalgebra of an ultraproduct algebra.

A tracial von Neumann algebra $(M, \tau)$ is called amenable if there exists a net $\xi_{n} \in L^{2}(M) \bar{\otimes} L^{2}(M)$ such that $\left\langle x \xi_{n}, \xi_{n}\right\rangle \rightarrow \tau(x)$ and $\left\|x \xi_{n}-\xi_{n} x\right\|_{2} \rightarrow 0$, for every $x \in M$. By Connes' celebrated theorem [Co76], $M$ is amenable if and only if it is approximately finite dimensional.

For a free ultrafilter $\omega$ on $\mathbb{N}$, the ultraproduct algebra $M^{\omega}$ is defined as the quotient $\ell^{\infty}(\mathbb{N}, M) / \mathcal{I}$, where $\mathcal{I} \subset \ell^{\infty}(\mathbb{N}, M)$ is the closed ideal of $x=\left(x_{n}\right)_{n}$ such that $\lim _{n \rightarrow \omega}\left\|x_{n}\right\|_{2}=0$. As it turns out, $M^{\omega}$ is a tracial von Neumann algebra, with its canonical trace given by $\tau_{\omega}\left(\left(x_{n}\right)_{n}\right)=\lim _{n \rightarrow \omega} \tau\left(x_{n}\right)$.

If $M$ and $N$ are tracial von Neumann algebras, then an $M-N$ bimodule is a Hilbert space $\mathcal{H}$ endowed with commuting normal $*$-homomorphisms $\pi: M \rightarrow \mathbb{B}(\mathcal{H})$ and $\rho: N^{o p} \rightarrow \mathbb{B}(\mathcal{H})$. For $x \in M, y \in N$ and $\xi \in \mathcal{H}$ we denote $x \xi y=\pi(x) \rho(y)(\xi)$. If $M, N, P$ are tracial von Neumann algebras, and $\mathcal{H}$ and $\mathcal{K}$ are $M-N$ and $N-P$ bimodules, respectively, then $\mathcal{H} \otimes_{N} \mathcal{K}$ denotes the Connes tensor product endowed with the natural $M-P$ bimodule structure (see [Po86]).

Let $Q \subset M$ be a von Neumann subalgebra. Jones' basic construction $\left\langle M, e_{Q}\right\rangle$ is defined as the von Neumann subalgebra of $\mathbb{B}\left(L^{2}(M)\right)$ generated by $M$ and the orthogonal projection $e_{Q}$ from $L^{2}(M)$ onto $L^{2}(Q)$. Recall that $\left\langle M, e_{Q}\right\rangle$ has a faithful semi-finite trace given by $\operatorname{Tr}\left(x e_{Q} y\right)=\tau(x y)$ for all $x, y \in M$. We denote by $L^{2}\left(\left\langle M, e_{Q}\right\rangle\right)$ the associated Hilbert space and endow it with the natural $M$-bimodule structure. Note that $L^{2}\left(\left\langle M, e_{Q}\right\rangle\right) \cong L^{2}(M) \otimes_{Q} L^{2}(M)$, as $M$ - $M$ bimodules.

Finally, if $S$ is a subset of a von Neumann algebra $\mathcal{M}$, then a state $\phi$ on $\mathcal{M}$ is said to be $S$-central if it satisfies $\phi(x T)=\phi(T x)$, for all $x \in S$ and $T \in \mathcal{M}$.

2.2. Intertwining-by-bimodules. We next recall from Po06b, Theorem 2.1 and Corollary 2.3] Popa's powerful intertwining-by-bimodules technique (see also Va07. Appendix C]). 
Theorem 2.1 ([Po06b]). Let $(M, \tau)$ be a separable tracial von Neumann algebra and $P, Q \subset M$ be two (not necessarily unital) von Neumann subalgebras. Then the following are equivalent:

- There exist non-zero projections $p \in P, q \in Q$, a*-homomorphism $\phi$ : $p P p \rightarrow q Q q$ and a non-zero partial isometry $v \in q M p$ such that $\phi(x) v=v x$, for all $x \in p P p$.

- There is no sequence $u_{n} \in \mathcal{U}(P)$ satisfying $\left\|E_{Q}\left(x u_{n} y\right)\right\|_{2} \rightarrow 0$, for all $x, y \in$ $M$.

If one of these conditions holds true, then we say that a corner of $P$ embeds into $Q$ inside $M$ and write $P \prec_{M} Q$.

If $M$ is not separable, then this statement holds true after we replace the sequence $u_{n}$ with a net.

\subsection{Relative amenability.}

Definition 2.2 ([OP10, Definition 2.2]). Let $(M, \tau)$ be a tracial von Neumann algebra and let $P \subset p M p, Q \subset M$ be von Neumann subalgebras. We say that $P$ is amenable relative to $Q$ inside $M$ if there exists a net $\xi_{n} \in L^{2}\left(p\left\langle M, e_{Q}\right\rangle p\right)$ such that $\left\langle x \xi_{n}, \xi_{n}\right\rangle \rightarrow \tau(x)$, for every $x \in p M p$, and $\left\|y \xi_{n}-\xi_{n} y\right\|_{2} \rightarrow 0$, for every $y \in P$. By OP10, Theorem 2.1], this condition is equivalent to the existence of a $P$-central state $\phi$ on $p\left\langle M, e_{Q}\right\rangle p$ such that $\phi_{\mid p M p}=\tau_{\mid p M p}$.

Remark 2.3. Let $\Lambda<\Gamma$ be countable subgroups. By [AD95, Proposition 3.5], $L(\Gamma)$ is amenable relative to $L(\Lambda)$ if and only if $\Lambda$ is co-amenable in $\Gamma$ : there is a $\Gamma$-invariant state on $\ell^{\infty}(\Gamma / \Lambda)$.

The failure of an algebra to be amenable (or amenable relative to some other algebra) can therefore be viewed as a source of "spectral gap rigidity". The notion of spectral gap rigidity has been introduced by Popa and has been used to great effect for instance in Po08, Po07a, OP10. Motivated by this, we introduce the following:

Definition 2.4. Let $(M, \tau)$ be a tracial von Neumann algebra and $P, Q \subset M$ be von Neumann subalgebras.

(1) A finite set $S \subset M$ is called a non-amenability set for $M$ if there exists a constant $K>0$ such that $\|\xi\|_{2} \leqslant K \sum_{y \in S}\|y \xi-\xi y\|_{2}$, for every $\xi \in$ $L^{2}(M) \bar{\otimes} L^{2}(M)$.

(2) A finite set $S \subset P$ is called a non-amenability set for $P$ relative to $Q$ inside $M$ if there exists a constant $K>0$ such that $\|\xi\|_{2} \leqslant K \sum_{y \in S}\|y \xi-\xi y\|_{2}$, for every $\xi \in L^{2}\left(\left\langle M, e_{Q}\right\rangle\right)$.

Remark 2.5. If $M$ has a non-amenability set, then $M$ has no amenable direct summand. If $M$ is a $\mathrm{II}_{1}$ factor, then by Connes' theorem Co76 the converse is true: $M$ admits a non-amenability set if and only if it is non-amenable. Note also that if there is a non-amenability set for $P$ relative to $Q$, then $P p$ is not amenable relative to $Q$, for any projection $p \in P^{\prime} \cap M$.

We will need the following result later, which is an easy consequence of OP10, Section 2.2] (see also [PV14a, Section 2.5]). 
Lemma 2.6 ([P10 $)$. Let $(M, \tau)$ be a tracial von Neumann algebra and let $P, Q \subset$ $M$ be von Neumann subalgebras. Let $\mathcal{H}$ be a $Q-P$ bimodule.

(1) Assume that $P p$ is not amenable relative to $Q$, for any non-zero projection $p \in P^{\prime} \cap M$. Then for any net of vectors $\xi_{n} \in L^{2}(M) \otimes_{Q} \mathcal{H}$ satisfying $\sup _{n}\left\|x \xi_{n}\right\| \leqslant\|x\|_{2}$, for all $x \in M$, and $\left\|y \xi_{n}-\xi_{n} y\right\| \rightarrow 0$, for all $y \in P$, we have that $\left\|\xi_{n}\right\| \rightarrow 0$.

(2) If $S \subset P$ is a non-amenability set for $P$ relative to $Q$ inside $M$, then there exists a constant $\kappa>0$ such that $\|\xi\| \leqslant \kappa \sum_{y \in S}\|y \xi-\xi y\|$, for all $\xi \in L^{2}(M) \otimes_{Q} \mathcal{H}$.

Proof. The first assertion is a rephrasing of [Io15, Lemma 2.3].

To prove the second assertion, let $S$ be a non-amenability set for $P$ relative to $Q$. Assuming that the conclusion fails, we can find a sequence of unit vectors $\xi_{n} \in L^{2}(M) \otimes_{Q} \mathcal{H}$ such that $\left\|y \xi_{n}-\xi_{n} y\right\| \rightarrow 0$, for all $y \in S$. Choose a state on $\ell^{\infty}(\mathbb{N})$, denoted $\lim _{n}$, extending the usual limit. Also, consider the normal $*-$ homomorphism $\pi:\left\langle M, e_{Q}\right\rangle \rightarrow \mathbb{B}\left(L^{2}(M) \otimes_{Q} \mathcal{H}\right)$ given by $\pi(T)\left(\xi \otimes_{Q} \eta\right)=T(\xi) \otimes_{Q} \eta$.

Define $\psi:\left\langle M, e_{Q}\right\rangle \rightarrow \mathbb{C}$ by letting $\psi(T)=\lim _{n}\left\langle\pi(T) \xi_{n}, \xi_{n}\right\rangle$. Then $\psi$ is an $S$-central state. Moreover, $\psi$ is $C^{*}(S)$-central, where $C^{*}(S)$ denotes the $C^{*}$ algebra generated by $S$. A standard procedure (see the proof of OP10, Theorem 2.1]) implies the existence of a net of unit vectors $\eta_{i} \in L^{2}\left(\left\langle M, e_{Q}\right\rangle\right)$ such that $\left\|y \eta_{i}-\eta_{i} y\right\| \rightarrow 0$, for all $y \in \mathcal{U}\left(C^{*}(S)\right)$. Thus, $\left\|y \eta_{i}-\eta_{i} y\right\| \rightarrow 0$, for all $y \in S$, contradicting the non-amenability of $S$.

The next lemma is a variant of Popa's spectral gap argument Po08. It will be used later (e.g. in the proof of Theorem 1.1) to deduce boundedness of a derivation $\delta$ from the uniform convergence of the semigroup $\phi_{t}=\exp \left(-t \delta^{*} \bar{\delta}\right)$.

Lemma 2.7. Let $(\tilde{M}, \tau)$ be a tracial von Neumann algebra and $M$ be a von Neumann subalgebra. Let $P, Q \subset M$ be von Neumann subalgebras. Assume that the $M-M$ bimodule $L^{2}(\tilde{M}) \ominus L^{2}(M)$ is isomorphic to $L^{2}(M) \otimes_{Q} \mathcal{K}$, for some $Q-M$ bimodule $\mathcal{K}$.

Let $\alpha_{n}: M \rightarrow \tilde{M}, n \geqslant 1$, be trace preserving *-homomorphisms such that $\left\|\alpha_{n}(x)-x\right\|_{2} \rightarrow 0$, for all $x \in M$. Assume that $p_{n} \in \alpha_{n}(P)^{\prime} \cap \tilde{M}$ is a projection and $v_{n} \in \tilde{M}$ is a unitary such that $\alpha_{n}(P) p_{n} \subset v_{n} M v_{n}{ }^{*}$, for all $n \geqslant 1$.

(1) If $P p$ is not amenable relative to $Q$ inside $M$, for every non-zero projection $p \in P^{\prime} \cap M$, then $\sup _{x \in(P)_{1}}\left\|\left(\alpha_{n}(x)-E_{M}\left(\alpha_{n}(x)\right)\right) p_{n}\right\|_{2} \rightarrow 0$, as $n \rightarrow \infty$.

(2) If $S \subset P$ is a non-amenability set for $P$ relative to $Q$ inside $M$, then there exists a constant $C>0$ such that for all $n \geqslant 1$ we have

$$
\left\|\left(\alpha_{n}(x)-E_{M}\left(\alpha_{n}(x)\right)\right) p_{n}\right\|_{2} \leqslant C \sum_{y \in S}\left\|\left(\alpha_{n}(y)-y\right) p_{n}\right\|_{2}, \text { for all } x \in(P)_{1} .
$$

Proof. For $x \in P$ and $n \geqslant 1$, define $\beta_{n}(x)=v_{n}^{*} \alpha_{n}(x) p_{n} v_{n} \in M$. Denote $\mathcal{H}=$ $L^{2}(\tilde{M}) \ominus L^{2}(M)$. Let $\mathcal{H}_{n}$ be the $M-P$ bimodule which is equal to $\mathcal{H}$ endowed with the bimodule structure given by $y \cdot \xi \cdot x=y \xi \beta_{n}(x)$. Then $\mathcal{H}_{n}$ is isomorphic to $L^{2}(M) \otimes_{Q} \mathcal{K}_{n}$, where $\mathcal{K}_{n}$ is equal to $\mathcal{K}$ endowed with the $Q-P$ bimodule structure given by $y \cdot \xi \cdot x=y \xi \beta_{n}(x)$.

Define the $Q$-P bimodule $\tilde{\mathcal{K}}=\bigoplus_{n \geqslant 1} \mathcal{K}_{n}$. Next, we treat separately the two assertions.

(1) In this case, Lemma 2.6 (1) implies that any net $\xi_{n} \in L^{2}(M) \otimes_{Q} \tilde{\mathcal{K}}$ satisfying $\left\|x \cdot \xi_{n}\right\| \leqslant\|x\|_{2}$ for every $x \in M$, and $\left\|x \cdot \xi_{n}-\xi_{n} \cdot x\right\| \rightarrow 0$, for all $x \in P$, must verify 
$\left\|\xi_{n}\right\| \rightarrow 0$. Define $\xi_{n}=p_{n} v_{n}-E_{M}\left(p_{n} v_{n}\right)$; then $\left\|x \xi_{n}\right\|_{2} \leqslant\|x\|_{2}$, for all $x \in M$. If we view $\xi_{n}$ as an element of $\mathcal{H}_{n}$; then for every $x \in P$ we have that

$$
\begin{aligned}
\left\|x \cdot \xi_{n}-\xi_{n} \cdot x\right\| & =\left\|x \xi_{n}-\xi_{n} \beta_{n}(x)\right\|_{2} \leqslant\left\|x p_{n} v_{n}-p_{n} v_{n} \beta_{n}(x)\right\|_{2} \\
& =\left\|\left(x-\alpha_{n}(x)\right) p_{n} v_{n}\right\|_{2} \rightarrow 0 .
\end{aligned}
$$

Since $\mathcal{H}_{n}$ is isomorphic to a $M-P$ sub-bimodule of $L^{2}(M) \otimes_{Q} \tilde{\mathcal{K}}$, we conclude that $\left\|\xi_{n}\right\|_{2} \rightarrow 0$. It follows that for all $x \in(P)_{1}$ and $n \geqslant 1$ we have

$$
\begin{gathered}
\left\|\alpha_{n}(x) p_{n}-E_{M}\left(\alpha_{n}(x)\right) p_{n}\right\|_{2}=\left\|\alpha_{n}(x) p_{n} v_{n}-E_{M}\left(\alpha_{n}(x)\right) p_{n} v_{n}\right\|_{2} \\
\leqslant 2\left\|\xi_{n}\right\|_{2}+\left\|\left(1-E_{M}\right)\left(\alpha_{n}(x) p_{n} v_{n}\right)\right\|_{2}=2\left\|\xi_{n}\right\|_{2}+\left\|\left(1-E_{M}\right)\left(p_{n} v_{n} \beta_{n}(x)\right)\right\|_{2} \\
\leqslant 2\left\|\xi_{n}\right\|_{2}+\left\|\left(1-E_{M}\right)\left(p_{n} v_{n}\right)\right\|_{2}=3\left\|\xi_{n}\right\|_{2} .
\end{gathered}
$$

Since $\left\|\xi_{n}\right\|_{2} \rightarrow 0$ and $x \in(P)_{1}$ is arbitrary, this proves the first assertion.

(2) Assume that $S$ is a non-amenability set for $P$ relative to $B$. Lemma 2.6 (2) implies that we can find $\kappa>0$ such that any vector $\xi \in L^{2}(M) \otimes_{B} \tilde{\mathcal{K}}$ verifies $\|\xi\| \leqslant \kappa \sum_{y \in S}\|y \cdot \xi-\xi \cdot y\|$. Thus, for all $n \geqslant 1$ and $\xi \in L^{2}(\tilde{M}) \ominus L^{2}(M)$, we have that $\|\xi\|_{2} \leqslant \kappa \sum_{y \in S}\left\|y \xi-\xi \beta_{n}(y)\right\|_{2}$.

Denote $\delta_{n}=\sum_{y \in S}\left\|\left(\alpha_{n}(y)-y\right) p_{n}\right\|_{2}$. Then we have $\sum_{y \in S}\left\|y p_{n} v_{n}-p_{n} v_{n} \beta_{n}(y)\right\|_{2}$ $=\delta_{n}$ and thus

$$
\sum_{y \in S}\left\|y\left(p_{n} v_{n}-E_{M}\left(p_{n} v_{n}\right)\right)-\left(p_{n} v_{n}-E_{M}\left(p_{n} v_{n}\right)\right) \beta_{n}(y)\right\|_{2} \leqslant \delta_{n} .
$$

By combining the last two inequalities we conclude that $\left\|p_{n} v_{n}-E_{M}\left(p_{n} v_{n}\right)\right\|_{2} \leqslant \kappa \delta_{n}$, for all $n \geqslant 1$. Together with the estimate from the proof of part (1), we get that if $x \in(P)_{1}$ and $n \geqslant 1$, then $\left\|\left(\alpha_{n}-E_{M}\left(\alpha_{n}(x)\right)\right) p_{n}\right\|_{2} \leqslant 3\left\|p_{n} v_{n}-E_{M}\left(p_{n} v_{n}\right)\right\|_{2} \leqslant 3 \kappa \delta_{n}$. Thus, the second assertion holds for $C=3 \kappa$.

2.4. Property Gamma. A $\mathrm{II}_{1}$ factor $M$ has property Gamma of Murray and von Neumann [MvN43] if there exists a sequence of unitaries $u_{n} \in M$ with $\tau\left(u_{n}\right)=0$ such that $\left\|u_{n} x-x u_{n}\right\|_{2} \rightarrow 0$, for all $x \in M$. If $\omega$ is a free ultrafilter on $\mathbb{N}$, then property Gamma is equivalent to $M^{\prime} \cap M^{\omega}=\mathbb{C} 1$. By a well-known result of Connes [Co76. Theorem 2.1] property Gamma is also equivalent to the existence of a net of unit vectors $\xi_{n} \in L^{2}(M) \ominus \mathbb{C} 1$ satisfying $\left\|x \xi_{n}-\xi_{n} x\right\|_{2} \rightarrow 0$, for all $x \in M$.

Therefore, the failure of property Gamma implies the existence of a non-Gamma set in the sense of the following definition that was introduced in $[\mathrm{Pe} 09 \mathrm{a}$, Definition 3.1] and motivated by [Po86, Remark 4.1.6].

Definition 2.8 ([Pe09a $)$. Let $M$ be a $\mathrm{II}_{1}$ factor. A finite set $S \subset M$ is called a non-Gamma set for $M$ if there exists $K>0$ such that $\|\xi\|_{2} \leqslant \sum_{y \in S} K\|y \xi-\xi y\|_{2}$, for all $\xi \in L^{2}(M) \ominus \mathbb{C} 1$.

Remark 2.9. By Co76, Theorem 2.1] any $\mathrm{II}_{1}$ factor $M$ without property Gamma has a non-Gamma set. Note, however, that it is not always possible to find a nonGamma set for $M$ inside a given weakly dense *-subalgebra of $M$. Recall that a countable group $\Gamma$ is inner amenable if the unitary representation of $\Gamma$ on $\ell^{2}(\Gamma \backslash\{e\})$ given by conjugation has almost invariant vectors. Vaes recently found an example of an icc group $\Gamma$ which is inner amenable (hence $\mathbb{C} \Gamma$ does not contain a non-Gamma set for $L(\Gamma))$ such that $L(\Gamma)$ does not have property Gamma Va12.

The next result follows easily from [Co76], but for the reader's convenience we include a proof. 
Lemma 2.10 ([Co76]). Let $M$ be a $I I_{1}$ factor and $S \subset M$ be a finite set closed under adjoint.

If $S$ is a non-Gamma set for $M$, then $S$ is a non-amenability set for $M$.

Proof. Let $S$ be a non-Gamma set for $M$. Assume by contradiction that $S$ is not a non-amenability set. Thus, we can find a sequence of unit vectors $\xi_{n} \in$ $L^{2}(M) \bar{\otimes} L^{2}(M)$ such that $\left\|x \xi_{n}-\xi_{n} x\right\|_{2} \rightarrow 0$, for all $x \in S$. Choose a state on $\ell^{\infty}(\mathbb{N})$, denoted $\lim _{n}$, extending the usual limit. Define $\psi: \mathbb{B}\left(L^{2}(M)\right) \rightarrow \mathbb{C}$ by letting $\psi(T)=\lim _{n}\left\langle(T \otimes 1) \xi_{n}, \xi_{n}\right\rangle$.

Then $\psi$ is an $S$-central state; hence $\phi=\psi_{\mid M}: M \rightarrow \mathbb{C}$ is an $S$-central state. Moreover, $\phi$ is central under the $C^{*}$-algebra $C^{*}(S)$ generated by $S$. Let $\eta_{i} \in L^{1}(M)$ be a net of positive norm one elements such that $\tau\left(x \eta_{i}\right) \rightarrow \phi(x)$, for all $x \in M$. Since $\phi$ is $C^{*}(S)$-central, for all $u \in \mathcal{U}\left(C^{*}(S)\right)$ we have that $\tau\left(x\left(u \eta_{i} u^{*}-\eta_{i}\right)\right) \rightarrow 0$, for all $x \in M$. Thus, $u \eta_{i} u^{*}-\eta_{i} \rightarrow 0$, in the weak topology, for all $u \in \mathcal{U}\left(C^{*}(S)\right)$. The Hahn-Banach theorem implies that, after passing to convex combinations, we may assume that we have $\left\|u \eta_{i} u^{*}-\eta_{i}\right\|_{1} \rightarrow 0$ in addition to $\tau\left(x \eta_{i}\right) \rightarrow \phi(x)$, for every $x \in M$.

The Powers-Størmer inequality (see [BO08, Proposition 6.2.4]) gives that $\left\|u \eta_{i}^{1 / 2} u^{*}-\eta_{i}^{1 / 2}\right\|_{2} \rightarrow 0$, for all $u \in \mathcal{U}\left(C^{*}(S)\right)$. Hence $\left\|y \eta_{i}^{1 / 2}-\eta_{i}^{1 / 2} y\right\|_{2} \rightarrow 0$, for all $y \in S$. Since $S$ is a non-Gamma set, we derive that $\left\|\eta_{i}^{1 / 2}-c_{i} \cdot 1\right\|_{2} \rightarrow 0$, where $c_{i}=\left\langle\eta_{i}^{1 / 2}, 1\right\rangle$. Applying Powers-Størmer again yields that $\left\|\eta_{i}-c_{i}^{2} \cdot 1\right\|_{1} \rightarrow 0$. This implies that $c_{i}^{2} \tau(x) \rightarrow \phi(x)$, for all $x \in M$, hence $\phi=\tau$. Since $\psi$ is $C^{*}(S)$-central and $\psi_{\mid M}=\tau$, we get that $\psi$ is central under the von Neumann algebra $W^{*}(S)$ generated by $S$. Thus $W^{*}(S)$ is amenable, contradicting the fact that it is a $\mathrm{II}_{1}$ factor without property Gamma.

2.5. Mixing bimodules. Next, we recall the notion of mixing bimodules introduced in PS12, Definition 2.3].

Definition 2.11 ([PS12] $)$. Let $(M, \tau)$ be a tracial von Neumann algebra. We say that an $M-M$ bimodule $\mathcal{H}$ is mixing if for any sequence $a_{n} \in(M)_{1}$ such that $a_{n} \rightarrow 0$, weakly, we have

$$
\sup _{x \in(M)_{1}}\left|\left\langle a_{n} \xi x, \eta\right\rangle\right| \rightarrow 0 \text { and } \sup _{x \in(M)_{1}}\left|\left\langle x \xi a_{n}, \eta\right\rangle\right| \rightarrow 0 \text {, as } n \rightarrow \infty \text {, for all } \xi, \eta \in \mathcal{H} \text {. }
$$

The coarse $M-M$ bimodule $L^{2}(M) \bar{\otimes} L^{2}(M)$ is clearly mixing. Also, let $\Gamma$ be a countable group and $\pi: \Gamma \rightarrow \mathcal{U}(\mathcal{K})$ be a mixing unitary representation. Then it is easy to see that $\mathcal{H}=\mathcal{K} \otimes \ell^{2}(\Gamma)$ is a mixing $L(\Gamma)-L(\Gamma)$ bimodule (with its natural bimodule structure, defined as in [Po06a, 1.1.4.]).

2.6. Normalizers of subalgebras of amalgamated free product algebras. We will also need the following variant of [Io15, Theorem 1.6], which is a hybrid between Theorems 1.6 and 5.2 from Io15.

Theorem 2.12 (Io15). Let $\left(M_{1}, \tau_{1}\right)$ and $\left(M_{2}, \tau_{2}\right)$ be two tracial von Neumann algebras with a common von Neumann subalgebra $B$ such that $\tau_{1 \mid B}=\tau_{2 \mid B}$ and denote $M=M_{1} *_{B} M_{2}$. Let $(Q, \tau)$ be a tracial von Neumann algebra and $A \subset M \bar{\otimes} Q$ be an amenable von Neumann subalgebra. Denote by $P=\mathcal{N}_{M \bar{\otimes} Q}(A)^{\prime \prime}$ the von Neumann algebra generated by the normalizer of $A$ in $M \bar{\otimes} Q$. 
Assume that there are a group $\mathcal{U}$ and homomorphisms $\rho_{1}: \mathcal{U} \rightarrow \mathcal{U}(M), \rho_{2}: \mathcal{U} \rightarrow$ $\mathcal{U}(Q)$ such that

- $\rho_{1}(u) \otimes \rho_{2}(u) \in P$, for all $u \in \mathcal{U}$, and

- the von Neumann subalgebra $P_{0} \subset M$ generated by $\rho_{1}(\mathcal{U})$ satisfies $P_{0}^{\prime} \cap$ $M^{\omega}=\mathbb{C} 1$.

Then one of the following conditions holds true:

(1) $A \prec_{M \bar{\otimes} Q} B \bar{\otimes} Q$.

(2) $P_{0} \prec_{M} M_{i}$, for some $i \in\{1,2\}$.

(3) $P_{0}$ is amenable relative to $B$ inside $M$.

Proof. For completeness, let us briefly indicate how this result follows from [Io15].

Define $\mathcal{M}=M \bar{\otimes} Q, \mathcal{M}_{1}=M_{1} \bar{\otimes} Q, \mathcal{M}_{2}=M_{2} \bar{\otimes} Q$ and $\mathcal{B}=B \bar{\otimes} Q$. Then $\mathcal{M}=\mathcal{M}_{1} *_{\mathcal{B}} \mathcal{M}_{2}$. Further, we define $\tilde{\mathcal{M}}=\mathcal{M} *_{\mathcal{B}}\left(\mathcal{B} \otimes L\left(\mathbb{F}_{2}\right)\right)$ and let $\left\{\theta_{t}\right\}_{t \in \mathbb{R}} \subset$ $\operatorname{Aut}(\tilde{\mathcal{M}})$ be the free malleable deformation [IPP08] (see e.g. [Io15, Section 2.5]). Let $\left\{u_{g}\right\}_{g \in \mathbb{F}_{2}}$ denote the canonical unitaries and define $N \subset \tilde{\mathcal{M}}$ to be the von Neumann subalgebra generated by $\bigcup_{g \in \mathbb{F}_{2}} u_{g} \mathcal{M} u_{g}^{*}$. Then $N$ is normalized by $\left\{u_{g}\right\}_{g \in \mathbb{F}_{2}}$ and $\tilde{\mathcal{M}}=N \rtimes \mathbb{F}_{2}$.

Now, notice that if $t \in(0,1)$, then $\theta_{t}(P) \subset \mathcal{N}_{\tilde{\mathcal{M}}}\left(\theta_{t}(A)\right)$. Popa and Vaes' dichotomy theorem [PV14a, Theorem 1.6] implies that either $\theta_{t}(A) \prec_{\tilde{\mathcal{M}}} N$ or $\theta_{t}(P)$ is amenable relative to $N$. By letting $t \in(0,1)$ vary, we conclude that we are in one of the following two cases:

Case 1. $\theta_{t}(A) \prec_{\tilde{\mathcal{M}}} N$, for some $t \in(0,1)$.

Case 2. $\theta_{t}(P)$ is amenable relative to $N$, for all $t \in(0,1)$.

In the first case, [Io15, Theorem 3.2] implies that either $A \prec_{\mathcal{M}} \mathcal{B}$ or $P \prec_{\mathcal{M}} \mathcal{M}_{i}$, for some $i \in\{1,2\}$. If the first alternative holds, then (1) is true. If $P \prec \mathcal{M} \mathcal{M}_{i}$, then $P_{0} \prec_{M} M_{i}$ and hence (2) is true. Indeed, if $P_{0} \nprec_{M} M_{i}$, then by the proof of $\left[\mathrm{Po} 06 \mathrm{~b}\right.$, Corollary 2.3] we can find a sequence of unitaries $u_{n} \in \mathcal{U}$ such that $\left\|E_{M_{i}}\left(a \rho_{1}\left(u_{n}\right) b\right)\right\|_{2} \rightarrow 0$, for all $a, b \in M$. But then it is clear that

$$
\left\|E_{\mathcal{M}_{i}}\left(a\left(\rho_{1}\left(u_{n}\right) \otimes \rho_{2}\left(u_{n}\right)\right) b\right)\right\|_{2} \rightarrow 0, \quad \text { for all } a, b \in \mathcal{M} .
$$

This contradicts the assumption that $P \prec \mathcal{M} \mathcal{M}_{i}$.

In the second case, [I015, Theorem 5.2] directly implies that either (2) or (3) holds.

\section{Derivations AND Free Dilations}

In this section we record several results about derivations and their dilations.

Let $(M, \tau)$ be a tracial von Neumann algebra, $M_{0} \subset M$ a weakly dense *subalgebra, and $\mathcal{H}$ an $M-M$ bimodule. A map $\delta: M_{0} \rightarrow \mathcal{H}$ is a derivation if $\delta(x y)=x \delta(y)+\delta(x) y$, for all $x, y \in M_{0}$. We assume that $\delta$ is closable as an unbounded operator $\delta: L^{2}(M) \rightarrow \mathcal{H}$. We also suppose that $\delta$ is real; i.e. there exists a conjugate-linear isometric involution $\mathcal{J}$ on $\mathcal{H}$ satisfying $\mathcal{J}(x \delta(y) z)=z^{*} \delta\left(y^{*}\right) x^{*}$, for all $x, y, z \in M_{0}$. When $\mathcal{H}=L^{2}(\mathcal{M})$, for some semifinite von Neumann algebra $\mathcal{M}$ containing $M$, we assume that $\mathcal{J}$ is given by $\mathcal{J}(x)=x^{*}$. In this case, $\delta$ is real if and only if $\delta\left(x^{*}\right)=\delta(x)^{*}$, for all $x \in M$.

Now, denote by $\bar{\delta}$ the closure of $\delta$ and by $D(\bar{\delta}) \subset L^{2}(M)$ the domain of $\bar{\delta}$. By [Sa89] and DL92], $D(\bar{\delta}) \cap M$ is a $*$-subalgebra and $\bar{\delta}_{\mid D(\bar{\delta}) \cap M}$ is a derivation. Further, $\Delta=\delta^{*} \bar{\delta}$ gives rise to a semigroup of completely positive maps on $M$. 
More precisely, $\phi_{t}=\exp (-t \Delta): M \rightarrow M$ are unital, trace preserving, completely positive maps satisfying $\phi_{t} \circ \phi_{s}=\phi_{t+s}$, for all $t, s>0$, and $\left\|\phi_{t}(x)-x\right\|_{2} \rightarrow 0$, as $t \rightarrow 0$, for every $x \in M$. Additionally, since $\delta$ is real, we have that $\phi_{t}$ is symmetric for every $t>0: \tau\left(\phi_{t}(x) y\right)=\tau\left(x \phi_{t}(y)\right)$, for all $x, y \in M$.

Recently it was proved that the semigroup $\left\{\phi_{t}\right\}_{t>0}$ admits a dilation in a larger tracial von Neumann algebra $\tilde{M} \supset M$, for any real closable derivation $\delta$ (see Da10b, Theorem 24]). Here, we state this result in the case when $\mathcal{H}$ is a multiple of the coarse $M-M$ bimodule. In this case, Da10b, Proposition 26] provides additional information on certain sub-bimodules of $L^{2}(\tilde{M})$.

Theorem 3.1 (Da10b] $)$. Let $(M, \tau)$ be a tracial von Neumann algebra and $M_{0} \subset$ $M$ be a weakly dense $*$-subalgebra. Let $\delta: M_{0} \rightarrow\left(L^{2}(M) \bar{\otimes} L^{2}(M)\right)^{\oplus \infty}$ be a real closable derivation. Let $\Delta=\delta^{*} \bar{\delta}$ and consider the semigroup of completely positive maps $\phi_{t}=\exp (-t \Delta): M \rightarrow M$.

Then there exists a tracial von Neumann algebra $\tilde{M}$ which contains $M$ and *homomorphisms $\alpha_{t}: M \rightarrow \tilde{M}$ such that $\phi_{t}=E_{M} \circ \alpha_{t}$, for all $t>0$. Moreover, denote by $M_{t} \subset \tilde{M}$ the von Neumann subalgebra generated by $M$ and $\alpha_{t}(M)$. Then the $M-M$ bimodule $L^{2}\left(M_{t}\right) \ominus L^{2}(M)$ is isomorphic to a sub-bimodule of $\left(L^{2}(M) \bar{\otimes} L^{2}(M)\right)^{\oplus \infty}$, for every $t>0$.

Later on, we will also need the following technical result.

Lemma 3.2. Consider the notation from Theorem 3.1. Then for every $x \in D(\bar{\delta})$ we have that

$$
\frac{1}{t}\left\|\alpha_{t}(x)-\phi_{t}(x)\right\|_{2}^{2} \rightarrow 2\|\delta(x)\|_{2}^{2} \quad \text { and } \quad \frac{1}{t}\left\|\alpha_{t}(x)-x\right\|_{2}^{2} \rightarrow 2\|\delta(x)\|_{2}^{2}, \quad \text { as } \quad t \rightarrow 0 .
$$

Proof. Let $t>0$ and recall that we have $\phi_{t}=\exp (-t \Delta)$, where $\Delta=\delta^{*} \bar{\delta}$. By combining the identity id $-\phi_{t}=\int_{0}^{t} \Delta \circ \phi_{s} \mathrm{~d} s$ with the fact that $x \in D(\bar{\delta})=D\left(\Delta^{1 / 2}\right)$, we get that

$$
\left\langle x-\phi_{t}(x), x\right\rangle=\int_{0}^{t}\left\langle\Delta\left(\phi_{s}(x)\right), x\right\rangle \mathrm{d} s=\int_{0}^{t}\left\langle\phi_{s}\left(\Delta^{1 / 2}(x)\right), \Delta^{1 / 2}(x)\right\rangle \mathrm{d} s .
$$

Since $\phi_{s}\left(\Delta^{1 / 2}(x)\right) \rightarrow \Delta^{1 / 2}(x)$, in $\|\cdot\|_{2}$, as $s \rightarrow 0$, we conclude that

$$
\frac{1}{t}\left\langle x-\phi_{t}(x), x\right\rangle \rightarrow\left\|\Delta^{1 / 2}(x)\right\|_{2}^{2}=\|\delta(x)\|_{2}^{2}, \text { as } t \rightarrow 0 .
$$

Finally, since $\phi_{t}(x)=E_{M}\left(\alpha_{t}(x)\right)$, we get that $\left\|\alpha_{t}(x)-\phi_{t}(x)\right\|_{2}^{2}=\|x\|_{2}^{2}-$ $\left\|\phi_{t}(x)\right\|_{2}^{2}=\left\langle x-\phi_{2 t}(x), x\right\rangle$. Also, we have that $\left\|\alpha_{t}(x)-x\right\|_{2}^{2}=2\left\langle x-\phi_{t}(x), x\right\rangle$. Together with equation (3.1) these identities yield the conclusion.

In the next section, the dilations from Theorem 3.1 will be used to prove that certain $\mathrm{II}_{1}$ factors $M$ are prime. On the other hand, in order to deduce that $M$ does not have Cartan subalgebras, we will additionally need to know that the dilation "lives" in the free product $\tilde{M}=M * L\left(\mathbb{F}_{\infty}\right)$. In the rest of this section, we recall two results in this direction.

Shlyakhtenko showed that any "algebraic" derivation $\delta: M_{0} \rightarrow L^{2}(M) \bar{\otimes} L^{2}(M)$ gives rise, via exponentiation, to a one-parameter group of automorphisms of $M *$ $L(\mathbb{Z})$ Sh09, Proposition 2]. Here, we note the following straightforward generalization of his result. 
Proposition 3.3. Let $(M, \tau)$ be a tracial von Neumann algebra, $B \subset M$ be a von Neumann subalgebra and $M_{0} \subset M$ be a weakly dense *-subalgebra. Assume that $\delta: D(\delta) \rightarrow L^{2}\left(\left\langle M, e_{B}\right\rangle\right)$ is a real derivation whose domain, denoted $D(\delta)$, contains both $B$ and $M_{0}$ such that

- $\delta\left(M_{0}\right) \subset \operatorname{span}\left(M_{0} e_{B} M_{0}\right)$.

- $e_{B}$ is in the domain of $\delta^{*}$ and $\delta^{*}\left(e_{B}\right) \in M_{0}$.

- $\delta(b)=0$, for all $b \in B$.

Assume that $M_{0}$ is finitely generated. More generally, assume that $M_{0}=$ $\bigcup_{n \geqslant 1} M_{n}$, where $M_{n}$ are finitely generated $*$-algebras satisfying $M_{n} \subset M_{n+1}$ and $\delta\left(M_{n}\right) \subset \operatorname{span}\left(M_{n} e_{B} M_{n}\right)$, for all $n \geqslant 1$.

Denote $\tilde{M}=M *_{B}(B \bar{\otimes} L(\mathbb{Z}))$ and let $s \in L(\mathbb{Z})$ be a generating $(0,1)$ semicircular element. Also, let $L^{2}\left(\left\langle M, e_{B}\right\rangle\right) \ni \xi \rightarrow \xi \# s \in \overline{\operatorname{span}(M s M)}^{\|\cdot\|_{2}} \subset L^{2}(\tilde{M})$ be the unique isomorphism of $M-M$ bimodules sending $e_{B}$ to $s$.

Then there exists a one-parameter group of automorphisms $\left\{\alpha_{t}\right\}_{t \in \mathbb{R}}$ of $\tilde{M}$ such that

$$
\left\|\frac{1}{t}\left(\alpha_{t}(x)-x\right)-\delta(x) \# s\right\|_{2} \rightarrow 0, \quad \text { as } t \rightarrow 0, \text { for all } x \in M_{0} .
$$

In this statement and below, we denote by $M s M$ the set $\{x s y \mid x, y \in M\}$.

The proof of Proposition 3.3 is an easy adaptation of the proof of [Sh09, Proposition 2] and can be derived by combining results from Sh00, Section 3]. Nevertheless, for the reader's convenience, we will sketch a proof.

Proof. If $b \in B$, then $\delta(b)=0$. This implies that $\delta^{*}\left(e_{B} b\right)=\delta^{*}\left(e_{B}\right) b$ and $\delta^{*}\left(b e_{B}\right)=$ $b \delta^{*}\left(e_{B}\right)$. Since $e_{B} b=b e_{B}$, we deduce that $\left[\delta^{*}\left(e_{B}\right), b\right]=0$.

Let $D(\tilde{\delta})$ be the weakly dense $*$-subalgebra of $\tilde{M}$ generated by $M_{0} \cup B \cup\{s\}$. Since $\delta(x) \# s \in D(\tilde{\delta})$, for all $x \in M_{0} \cup B$, and $\delta^{*}\left(e_{B}\right) \in M_{0}$, we can define $\tilde{\delta}$ : $M_{0} \cup B \cup\{s\} \rightarrow D(\tilde{\delta})$ by letting

$$
\tilde{\delta}(x)=\delta(x) \# s, \text { for all } x \in M_{0} \cup B \text {, and } \tilde{\delta}(s)=-\delta^{*}\left(e_{B}\right) .
$$

Since $\left[\delta^{*}\left(e_{B}\right), B\right]=0$ and $\delta_{\mid B} \equiv 0$, it is easy to see that $\tilde{\delta}$ extends to a derivation $\tilde{\delta}: D(\tilde{\delta}) \rightarrow D(\tilde{\delta})$. Also, since $\delta\left(x^{*}\right)=\delta(x)^{*}$, for all $x \in M_{0}$, and $\delta^{*}\left(e_{B}\right)$ is selfadjoint, we deduce that $\tilde{\delta}\left(x^{*}\right)=\tilde{\delta}(x)^{*}$, for all $x \in D(\tilde{\delta})$. Moreover, we have that:

Claim 1. $\tau(\tilde{\delta}(x))=0$, for all $x \in D(\tilde{\delta})$.

Proof of Claim 1. Denote by $\mathcal{M}$ the $*$-subalgebra of $M$ generated by $M_{0}$ and $B$. For $n \geqslant 1$, define $s_{n}=s^{n}-\tau\left(s^{n}\right)$. Then $D(\tilde{\delta})$ is the linear span of

$$
\mathcal{M} \cup\left\{x_{1} s_{n_{1}} x_{2} \ldots x_{k} s_{n_{k}} x_{k+1} \mid x_{1}, x_{k+1} \in \mathcal{M}, x_{2}, \ldots, x_{k} \in \mathcal{M} \ominus B, n_{1}, \ldots, n_{k} \geqslant 1\right\} .
$$

Since $\delta\left(M_{0}\right) \subset \operatorname{span}\left(M_{0} e_{B} M_{0}\right)$, we get that $\tilde{\delta}(\mathcal{M}) \subset \operatorname{span}(\mathcal{M} s \mathcal{M})$. Hence, $\tau(\tilde{\delta}(x))$ $=0$, for all $x \in \mathcal{M}$. Thus, in order to prove the claim, it suffices to show that $\tau(\tilde{\delta}(x))=0$, for every $x$ of the form $x=x_{1} s_{n_{2}} x_{2} \ldots x_{k} s_{n_{k}} x_{k+1}$, for some $k \geqslant 1$, $x_{1}, x_{k+1} \in \mathcal{M}, x_{2}, \ldots, x_{k} \in \mathcal{M} \ominus B$ and $n_{1}, \ldots, n_{k} \geqslant 1$.

Below, we sketch the proof of this fact in the case when $k$ is even, leaving the (similar) case when $k$ is odd to the reader. Assume therefore that $k=2 l$, for some $l \geqslant 1$.

Notice first that by freeness it follows that for all $i \in\{1, \ldots, k+1\} \backslash\{l\}, y \in$ $M L(\mathbb{Z}) M$ and every $j \in\{1, \ldots, k\} \backslash\{l, l+1\}, z \in L(\mathbb{Z}) M L(\mathbb{Z})$, we have that

$\tau\left(x_{1} s_{n_{1}} \ldots s_{n_{i}} y s_{n_{i+1}} \ldots s_{n_{k}} x_{k+1}\right)=0$ and $\tau\left(x_{1} s_{n_{1}} \ldots x_{n_{j}} z x_{n_{j+1}} \ldots s_{n_{k}} x_{k+1}\right)=0$. 
If $n \geqslant 1$, then

$$
\tilde{\delta}\left(s_{n}\right)=\tilde{\delta}\left(s^{n}\right)=\sum_{i=0}^{n-1} s^{i} \tilde{\delta}(s) s^{n-1-i} .
$$

Thus, $\tilde{\delta}\left(s_{n}\right) \in \operatorname{span}(L(\mathbb{Z}) M L(\mathbb{Z}))$. Also, recall that $\tilde{\delta}\left(x_{0}\right) \in \operatorname{span}(M L(\mathbb{Z}) M)$, for all $x_{0} \in \mathcal{M}$. By combining these facts with equation (3.2) and using Leibniz's rule for $\tilde{\delta}$, it follows that

$$
\tau(\tilde{\delta}(x))=\tau\left(x_{1} s_{n_{1}} \ldots x_{l} \tilde{\delta}\left(s_{n_{l}} x_{l+1} s_{n_{l+1}}\right) x_{l+2} \ldots s_{n_{k}} x_{k+1}\right) .
$$

Next, we denote by $\mathcal{K} \subset \tilde{M} \ominus B$ the set of alternating words in $M \ominus B$ and $L(\mathbb{Z}) \ominus \mathbb{C} 1$, which start or begin with an element from $L(\mathbb{Z}) \ominus \mathbb{C} 1$. Again, by freeness it is easy to see that

$$
\tau\left(x_{1} s_{n_{1}} \ldots x_{l} y x_{l+2} \ldots s_{n_{k}} x_{n_{k+1}}\right)=0 \text {, for all } y \in \mathcal{K} .
$$

Now, if $b \in B$, then $\tau(\tilde{\delta}(s) b)=\tau\left(\delta^{*}\left(e_{B}\right) b\right)=-\operatorname{Tr}\left(e_{B} \delta(b)\right)=0$ and thus $\tilde{\delta}(s) \in$ $M \ominus B$. In combination with the formula for $\tilde{\delta}\left(s_{n}\right)$, we derive that $\tilde{\delta}\left(s_{n_{l}}\right), \tilde{\delta}\left(s_{n_{l+1}}\right) \in$ $\operatorname{span}(L(\mathbb{Z})(M \ominus B) L(\mathbb{Z}))$. Also, since $x_{l+1} \in \mathcal{M}$, we have that $\tilde{\delta}\left(x_{l+1}\right) \in$ $\operatorname{span}(M(L(\mathbb{Z}) \ominus \mathbb{C} 1) M)$. Using these relations and Leibniz's rule it follows that $\tilde{\delta}\left(s_{n_{l}} x_{l+1} s_{n_{l+1}}\right)-\tau\left(\tilde{\delta}\left(s_{n_{l}} x_{l+1} s_{n_{l+1}}\right)\right)$ belongs to the linear span of $\mathcal{K}$.

Combining this fact with (3.3) and (3.4) yields that

$$
\tau(\tilde{\delta}(x))=\tau\left(\tilde{\delta}\left(s_{n_{l}} x_{l+1} s_{n_{l+1}}\right)\right) \tau\left(x_{1} s_{n_{1}} \ldots x_{l} x_{l+2} \ldots s_{n_{k}} x_{k+1}\right)
$$

and reduces Claim 1 to proving the following claim:

Claim 2. $\tau\left(\tilde{\delta}\left(s_{m} c s_{n}\right)\right)=0$, for all $m, n \geqslant 1$ and $c \in \mathcal{M} \ominus B$.

Proof of Claim 2. First, since $\tilde{\delta}\left(s_{n}\right)=\sum_{i=0}^{n-1} s^{i} \tilde{\delta}(s) s^{n-1-i}$, for all $n \geqslant 1$, we get that

$$
\tau\left(\tilde{\delta}\left(s_{m} c s_{n}\right)\right)=\sum_{i=0}^{m-1} \tau\left(s^{i} \tilde{\delta}(s) s^{m-1-i} c s_{n}\right)+\tau\left(s_{m} \tilde{\delta}(c) s_{n}\right)+\sum_{j=0}^{n-1} \tau\left(s_{m} c s^{j} \tilde{\delta}(s) s^{n-1-j}\right) .
$$

Note that $c, \tilde{\delta}(s) \in M \ominus B$ and $\tau(\tilde{\delta}(s) c)=-\tau\left(\delta^{*}\left(e_{B}\right) c\right)$. Since $\tilde{\delta}(c) \in \operatorname{span}(M s M)$, for every $x \in L(\mathbb{Z})$ we have that

$$
\begin{gathered}
\tau(\tilde{\delta}(c) x)=\tau(x s) \tau(\tilde{\delta}(c) s)=\tau(x s)\left\langle\delta(c) \# s, e_{B} \# s\right\rangle=\tau(x s)\left\langle\delta(c), e_{B}\right\rangle \\
=\tau(x s) \tau\left(c \delta^{*}\left(e_{B}\right)\right)=-\tau(\tilde{\delta}(s) c) \tau(x s) .
\end{gathered}
$$

Altogether, by combining equations (3.5) and (3.6) we get that

$\tau\left(\tilde{\delta}\left(s_{m} c s_{n}\right)\right)=\tau(\tilde{\delta}(s) c)\left[\sum_{i=0}^{m-1} \tau\left(s_{n} s^{i}\right) \tau\left(s^{m-1-i}\right)-\tau\left(s_{n} s_{m} s\right)+\sum_{j=0}^{n-1} \tau\left(s_{m} s^{j}\right) \tau\left(s^{n-1-j}\right)\right]$.

Now, let $\partial: \mathbb{C}\langle s\rangle \rightarrow \mathbb{C}\langle s\rangle \otimes \mathbb{C}\langle s\rangle$ be the difference quotient derivation given by $\partial(s)=1 \otimes 1$. Since $s$ is $(0,1)$ semicircular, we have that $\partial^{*}(1 \otimes 1)=s$ (see Vo98b, Proposition 3.8]) and hence $(\tau \otimes \tau)\left(\partial\left(s^{p}\right)\right)=\tau\left(s^{p+1}\right)$, for all $p$. Thus, we 
get that

$$
\begin{gathered}
\sum_{i=0}^{m-1} \tau\left(s_{n} s^{i}\right) \tau\left(s^{m-1-i}\right)+\sum_{j=0}^{n-1} \tau\left(s_{m} s^{j}\right) \tau\left(s^{n-1-j}\right) \\
=(\tau \otimes \tau)\left[s^{n} \partial\left(s^{m}\right)-\tau\left(s^{n}\right) \partial\left(s^{m}\right)+\partial\left(s^{n}\right) s^{m}-\tau\left(s^{m}\right) \partial\left(s^{n}\right)\right] \\
=\tau\left(s^{m+n+1}\right)-\tau\left(s^{n}\right) \tau\left(s^{m+1}\right)-\tau\left(s^{m}\right) \tau\left(s^{n+1}\right) .
\end{gathered}
$$

Since the last term is equal to $\tau\left(s_{n} s s_{m}\right)$, by using equation (3.7) we conclude that $\tau\left(\tilde{\delta}\left(s_{m} c s_{n}\right)\right)=0$. This finishes the proof of Claim 2 and hence of Claim 1.

Now, let $\tilde{M}_{0} \subset \tilde{M}$ be the $*$-subalgebra generated by $M_{0} \cup\{s\}$. Then $\tilde{M}_{0} \subset D(\tilde{\delta})$ and $\tilde{\delta}\left(\tilde{M}_{0}\right) \subset \tilde{M}_{0}$.

If $M_{0}$ is finitely generated, then $\tilde{M}_{0}$ is finitely generated. By using the fact that $\tilde{\delta}\left(x^{*}\right)=\tilde{\delta}(x)^{*}$, for all $x \in \tilde{M}_{0}$, and Claim 1, Vo02, Proposition 3.3 and Corollary 3.7] imply that $\tilde{\delta}$ exponentiates to a one-parameter group $\alpha_{t}=e^{t \tilde{\delta}}$ of trace preserving automorphisms of $\tilde{M}$. Moreover, $\alpha_{t}$ satisfies the convergence required in the conclusion.

Finally, assume that $M_{0}$ is the increasing union of *-algebras $M_{n}$ satisfying $\delta\left(M_{n}\right) \subset \operatorname{span}\left(M_{n} e_{B} M_{n}\right)$. Let $n_{0} \geqslant 1$ such that $\delta^{*}\left(e_{B}\right) \in M_{n_{0}}$. For every $n$, let $\tilde{M}_{n}$ be the *-algebra generated by $M_{n} \cup\{s\}$. Then for every $n \geqslant n_{0}$, we have that $\tilde{\delta}\left(\tilde{M}_{n}\right) \subset \tilde{M}_{n}$. By the above, $\alpha_{t}=e^{t \tilde{\delta}}$ defines a one-parameter group of trace preserving automorphisms of the weak closure of $\tilde{M}_{n}$. Since the increasing union $\bigcup_{n \geqslant 1} \tilde{M}_{n}$ is weakly dense in $\tilde{M}$, the conclusion follows.

Remark 3.4. Proposition 3.3 can be used to recover several known constructions of malleable deformations, in the sense of Popa. Let us give two such examples (see also Sh09, Example 1]).

(1) Let $M=M_{1} *_{B} M_{2}$ be an amalgamated free product of tracial von Neumann algebras. Denote by $D(\delta)$ the *-algebra generated by $M_{1}$ and $M_{2}$. Define a derivation $\delta: D(\delta) \rightarrow L^{2}\left(\left\langle M, e_{B}\right\rangle\right)$ by letting $\delta(x)=i\left[x, e_{B}\right]$ if $x \in M_{1}$, and $\delta(x)=0$ if $x \in M_{2}$. Then $\delta$ is real and satisfies the assumptions of Proposition 3.3 . For $t \in \mathbb{R}$, let $u_{t}=\exp ($ its $)$. The resulting one-parameter group of automorphisms of $\tilde{M}=M *_{B}(B \bar{\otimes} L(\mathbb{Z}))$ is given by $\alpha_{t}(x)=u_{t} x u_{t}^{*}$ if $x \in M_{1}$, and $\alpha_{t}(x)=x$ if $x \in M_{2} \cup L(\mathbb{Z})$. This is a variant of the free malleable deformation of $M$ introduced in IPP08.

(2) Let $Q \subset P$ be tracial von Neumann algebras and $\theta: Q \rightarrow P$ be a trace preserving $*$-homomorphism. From this data, an $\operatorname{HNN}$ extension $M=\operatorname{HNN}(P, Q, \theta)$ was constructed in Ue05] (see also [FV12, Section 3]). Briefly, $M$ is a tracial von Neumann algebra generated by $P$ and a unitary element $u$ such that $u x u^{*}=\theta(x)$, for all $x \in Q$. Denote by $D(\delta)$ the *-algebra generated by $P$ and $u$. Then it is easy to see that $\delta: D(\delta) \rightarrow L^{2}\left(\left\langle M, e_{Q}\right\rangle\right)$ given by $\delta(x)=0$ if $x \in P$, and $\delta(u)=i u e_{Q}$ defines a real derivation which satisfies the assumptions of Proposition 3.3. For $t \in \mathbb{R}$, let $v_{t}=\exp (i t s)$. Then the one-parameter group of automorphisms of $\tilde{M}=M *_{Q}(Q \bar{\otimes} L(\mathbb{Z}))$ provided by Proposition 3.3 satisfies $\alpha_{t}(x)=x$ if $x \in P \cup L(\mathbb{Z})$, and $\alpha_{t}(u)=u v_{t}$. This recovers the malleable deformation of $M$ introduced in [FV12, Section 3.5].

Remark 3.5. In view of Sh09, Proposition 2] and Proposition [3.3, it is natural to wonder which finitely generated $*$-algebras $A$ admit unbounded derivations 
$\delta: A \rightarrow A \otimes A$. In the case of group algebras, the existence of such derivations implies strong restrictions on the structure of the group. We are grateful to Jesse Peterson for pointing out this remark to us.

Let $\Gamma$ be an infinite, finitely generated countable group and assume that there exists an unbounded derivation $\delta: \mathbb{C} \Gamma \rightarrow \mathbb{C} \Gamma \otimes \mathbb{C} \Gamma$. Define $b: \Gamma \rightarrow \mathbb{C} \Gamma \otimes \mathbb{C} \Gamma$ by letting $b(g)=\delta\left(u_{g}\right) u_{g}^{*}$. Then we have that $b(g h)=b(g)+u_{g} b(h) u_{g}^{*}$, for all $g, h \in \Gamma$. Now, the representation of $\Gamma$ on $\mathbb{C} \Gamma \otimes \mathbb{C} \Gamma$ by conjugation is isomorphic to the left regular representation $\lambda$ of $\Gamma$ on $\bigoplus_{n=1}^{\infty} \mathbb{C} \Gamma$. Thus, we obtain a cocycle $c=\left(c_{n}\right): \Gamma \rightarrow \bigoplus_{n=1}^{\infty} \mathbb{C} \Gamma$.

Since $\delta$ is unbounded (hence not inner), it is easy to see that not all of the cocycles $c_{n}: \Gamma \rightarrow \mathbb{C} \Gamma$ can be inner. By [BV97, Lemma 2] this yields that $\Gamma$ has at least two ends. Stallings' theorem now implies that $\Gamma$ is either an amalgamated free product or an HNN extension over a finite subgroup. Thus, if $\Gamma$ is moreover torsion free, then it is the free product $\Gamma=\Gamma_{1} * \Gamma_{2}$ of two infinite groups.

We end this section by recalling [Da14, Corollary 25], which shows that under a Lipschitz conjugate variables condition, the von Neumann algebra $M$ generated by $n$ self-adjoint elements $X_{1}, \ldots, X_{n}$ admits a deformation into $M * L\left(\mathbb{F}_{\infty}\right)$.

Theorem 3.6 ([Da14] $)$. Let $(M, \tau)$ be a tracial von Neumann algebra generated by $n \geqslant 2$ self-adjoint elements $X_{1}, \ldots, X_{n}$. Let $M_{0}$ be the $*$-algebra generated by $X_{1}, \ldots, X_{n}$. For every $1 \leqslant i \leqslant n$, let $\delta_{i}: M_{0} \rightarrow L^{2}(M) \bar{\otimes} L^{2}(M)$ be the partial free difference quotient given by $\delta_{i}\left(X_{j}\right)=\delta_{i, j} X_{i}$. Denote $\delta=\left(\delta_{1}, \ldots, \delta_{n}\right): M_{0} \rightarrow$ $\left(L^{2}(M) \bar{\otimes} L^{2}(M)\right)^{\oplus_{n}}$ and let $\bar{\delta}$ be the closure of $\delta$.

Assume that $1 \otimes 1$ is in the domain of $\delta_{i}^{*}$ and denote by $\xi_{i}=\delta_{i}^{*}(1 \otimes 1)$ the corresponding conjugate variable. Moreover, assume that $\xi_{i}$ is in the domain of $\bar{\delta}$ and $\bar{\delta}\left(\xi_{i}\right) \in\left(M \bar{\otimes} M^{o p}\right)^{\oplus_{n}}$, for all $i \in\{1, \ldots, n\}$. Here, $M^{\text {op }}$ denotes the opposite algebra of $M$, and we consider the inclusion $M \bar{\otimes} M^{o p} \subset L^{2}\left(M \bar{\otimes} M^{o p}\right) \cong L^{2}(M) \bar{\otimes} L^{2}(M)$.

Then for every $t \geqslant 0$, there exists a free family $S_{1}^{(t)}, \ldots, S_{n}^{(t)} \in L\left(\mathbb{F}_{\infty}\right)$ of $(0,1)$ semicircular elements and a $*$-homomorphism $\alpha_{t}: M \rightarrow M * L\left(\mathbb{F}_{\infty}\right)$ such that

$$
\left\|\frac{1}{\sqrt{t}}\left(\alpha_{t}(x)-x\right)-\sum_{i=1}^{n} \delta_{i}(x) \# S_{i}^{(t)}\right\|_{2} \rightarrow 0, \text { as } t \rightarrow 0, \text { for all } x \in M_{0} \text {. }
$$

\section{4. $L^{2}$-RIGIDITY RESULTS}

The main goal of this section is to prove Theorem 1.1. Let us first recall Peterson's notion of $L^{2}$-rigidity for von Neumann algebras (see Pe09b, Definition 4.1 and Lemma 2.1]).

Definition 4.1 ([Pe09b] $)$. A tracial von Neumann algebra $(M, \tau)$ is $L^{2}$-rigid if for any densely defined real closable derivation $\delta: D(\delta) \rightarrow\left(L^{2}(M) \bar{\otimes} L^{2}(M)\right)^{\oplus \infty}$, the deformation $\phi_{t}=\exp \left(-t \delta^{*} \bar{\delta}\right)$ converges uniformly to $\operatorname{id}_{M}$ on $(M)_{1}$, as $t \rightarrow 0$.

4.1. Proof of Theorem [1.1. By Pe09b, Corollary 4.6] any non-amenable $\mathrm{II}_{1}$ factor that is non-prime or has property Gamma is $L^{2}$-rigid. Thus, in order to get the conclusion, it suffices to prove that $M$ is not $L^{2}$-rigid. Assume by contradiction that $M$ is $L^{2}$-rigid. 
Recall that $\delta: M_{0} \rightarrow L^{2}(M) \bar{\otimes} L^{2}(M)$ is a densely defined real derivation such that $M_{0}$ contains a non-amenability set $S$ for $M$. For every $t>0$, denote $\phi_{t}=$ $\exp \left(-t \delta^{*} \bar{\delta}\right)$. By Theorem 3.1 there exist a tracial von Neumann algebra $\tilde{M}$ containing $M$ and $*$-homomorphisms $\alpha_{t}: M \rightarrow \tilde{M}$ such that $\phi_{t}=E_{M} \circ \alpha_{t}$, for all $t>0$.

Since $M$ is $L^{2}$-rigid, $\phi_{t}$ converges uniformly to $\operatorname{id}_{M}$ on $(M)_{1}$. Thus, we can find $t_{0}>0$ such that $\left\|\phi_{t}(x)-x\right\|_{2} \leqslant \frac{1}{2}$, for all $t \in\left[0, t_{0}\right]$ and every $x \in(M)_{1}$. Fix $t \in\left[0, t_{0}\right]$. Then for every $u \in \mathcal{U}(M)$ we have that $\tau\left(\alpha_{t}(u) u^{*}\right)=\tau\left(\phi_{t}(u) u^{*}\right) \geqslant \frac{1}{2}$. Denote by $K \subset(\tilde{M})_{1}$ the $\|\cdot\|_{2}$-closure of the convex hull of the set $\left\{\alpha_{t}(u) u^{*} \mid u \in\right.$ $\mathcal{U}(M)\}$ and let $v_{t} \in K$ be the unique element of minimal $\|\cdot\|_{2}$. Then $\tau\left(v_{t}\right) \geqslant \frac{1}{2}$, hence $v_{t} \neq 0$, and $\alpha_{t}(u) v_{t}=v_{t} u$, for all $u \in \mathcal{U}(M)$.

Moreover, if we let $M_{t}$ be the von Neumann algebra generated by $\alpha_{t}(M)$ and $M$, then $v_{t} \in M_{t}$ and $v_{t}^{*} v_{t} \in M^{\prime} \cap M_{t}$. Thus, we get that $v_{t}^{*} v_{t}-E_{M}\left(v_{t}^{*} v_{t}\right) \in M^{\prime} \cap M_{t}$. On the other hand, Theorem 3.1 gives that the $M-M$ bimodule $L^{2}\left(M_{t}\right) \ominus L^{2}(M)$ is isomorphic to a sub-bimodule of $\left(L^{2}(M) \bar{\otimes} L^{2}(M)\right)^{\oplus \infty}$. Since $M$ is diffuse, we conclude that $v_{t}^{*} v_{t} \in M^{\prime} \cap M=\mathbb{C} 1$. Thus, by rescaling $v_{t}$, we get that there is a unitary $v_{t} \in M_{t}$ such that $\alpha_{t}(M) \subset v_{t} M v_{t}^{*}$, for every $t \in\left[0, t_{0}\right]$.

Let $t_{n} \in\left(0, t_{0}\right]$ be a sequence such that $t_{n} \rightarrow 0$. Since $S$ is a non-amenability set for $M$, by Lemma 2.7 (2) we can find a constant $C>0$ such that for all $n \geqslant 1$ we have

$$
\begin{aligned}
\left\|\alpha_{t_{n}}(x)-\phi_{t_{n}}(x)\right\|_{2} & =\left\|\alpha_{t_{n}}(x)-E_{M}\left(\alpha_{t_{n}}(x)\right)\right\|_{2} \\
& \leqslant C \sum_{y \in S}\left\|\alpha_{t_{n}}(y)-y\right\|_{2}, \text { for all } x \in(M)_{1} .
\end{aligned}
$$

Now, if we take $x \in M_{0}$, then Lemma 3.2 implies that $\frac{1}{\sqrt{t}}\left\|\alpha_{t}(x)-\phi_{t}(x)\right\|_{2} \rightarrow$ $\sqrt{2}\|\delta(x)\|_{2}$ and $\frac{1}{\sqrt{t}}\left\|\alpha_{t}(x)-x\right\|_{2} \rightarrow \sqrt{2}\|\delta(x)\|_{2}$, as $t \rightarrow 0$. In combination with the last inequality this gives that $\|\delta(x)\|_{2} \leqslant C \sum_{y \in S}\|\delta(y)\|_{2}$, for all $x \in M_{0}$. Thus, $\delta$ is bounded, which is a contradiction.

Remark 4.2. Let $M$ be a $\mathrm{II}_{1}$ factor and $p_{n} \in M$ a sequence of projections such that $\sum_{n=1}^{\infty} p_{n}=1$. Then $M_{0}=\bigcup_{n \geqslant 1}\left(p_{1}+\cdots+p_{n}\right) M\left(p_{1}+\cdots+p_{n}\right)$ is a weakly dense $*$-subalgebra of $M$. Let $\alpha_{n}$ be a sequence of positive real numbers such that $\sum_{n=1}^{\infty} \alpha_{n}^{2} \tau\left(p_{n}\right)^{2}=+\infty$. Then the map $\delta: M_{0} \rightarrow L^{2}(M) \bar{\otimes} L^{2}(M)$ given by $\delta(x)=\sum_{n=1}^{\infty} i \alpha_{n}\left[x, p_{n} \otimes p_{n}\right]$ is a well-defined derivation. Moreover, it is easy to see that $\delta$ is real, unbounded and closable. This shows that the assumption that $M_{0}$ contains a non-amenability set for $M$ is necessary in the hypothesis of Theorem 1.1 .

4.2. Proof of Corollary [1.2. We prove here Corollary 1.2 under the first assumption, and postpone dealing with the second assumption until Corollary 4.3 . Denote by $M_{0}$ the $*$-algebra generated by $X_{1}, \ldots, X_{n}$. For every $i \in\{1, \ldots, n\}$, let $\delta_{i}: M_{0} \rightarrow L^{2}(M) \bar{\otimes} L^{2}(M)$ be the partial free difference quotient derivation given by $\delta_{i}\left(X_{j}\right)=\delta_{i, j} 1 \otimes 1$.

Further, let $\delta=\left(\delta_{1}, \ldots, \delta_{n}\right): M_{0} \rightarrow\left(L^{2}(M) \bar{\otimes} L^{2}(M)\right)^{\oplus n}$. Since $\delta_{i}^{*}(1 \otimes 1)=$ $\mathcal{J}_{1}\left(X_{i}: \mathbb{C}\left\langle X_{1}, \ldots, \hat{X}_{i}, \ldots, X_{n}\right\rangle\right)$ exists and belongs to $L^{2}(M)$, Vo98b, Corollary 4.1] implies that $\delta_{i}$ is a closable derivation, for all $i \in\{1,2, \ldots, n\}$. Therefore, $\delta$ is real and closable. 
By [Da10a, Theorem 13], $M$ is a $\mathrm{II}_{1}$ factor without property Gamma. Moreover, since the first and second variables are bounded, Da10a, Lemmas 9 and 10] imply that $S=\left\{X_{1}, \ldots, X_{n}\right\}$ is a non-Gamma set for $M$ (see Da10a, Remark 11]). By Lemma 2.10 we therefore deduce that $S$ is a non-amenability set for $M$.

Since $\Phi^{*}\left(X_{1}, \ldots, X_{n}\right)=\sum_{i=1}^{n}\left\|\delta_{i}^{*}(1 \otimes 1)\right\|_{2}^{2}<\infty$, the distribution of $X_{i}$ has no atoms, for every $i$. Indeed, by Vo98b, Proposition 7.9], the free entropy $\chi^{*}$ satisfies $\chi^{*}\left(X_{1}, \ldots, X_{n}\right)>-\infty$. Since $\chi^{*}\left(X_{1}\right)+\cdots+\chi^{*}\left(X_{n}\right) \geqslant \chi^{*}\left(X_{1}, \ldots, X_{n}\right)$ (see Vo98b, Proposition 7.3]), we deduce that $\chi^{*}\left(X_{i}\right)>-\infty$, for all $i$. Finally, since $\chi^{*}\left(X_{i}\right)=\chi\left(X_{i}\right)$ (see [Vo98b, Proposition 7.6]), by Vo94, Proposition 4.5] we get that the distribution of $X_{i}$ has no atoms, for all $i \in\{1, \ldots, n\}$.

As in $[\mathrm{Pe} 09 \mathrm{a}$, Section 1.6] it follows that $\delta$ is not inner. Moreover, $\mathrm{Pe} 09 \mathrm{a}$, Theorem 2.2] implies that $\delta$ is unbounded. Altogether, we can apply Theorem 1.1 and deduce the conclusion.

Before we can state our next result, we need to recall some terminology. Let $(M, \tau)$ be a tracial von Neumann algebra generated by $n \geqslant 2$ algebraically free *-subalgebras $A_{1}, \ldots, A_{n}$. The next result uses the liberation Fisher information $\varphi^{*}\left(A_{1} ; \ldots ; A_{n}\right)$ introduced in [Vo99, Definition 9.1]. The definition involves the derivations $\delta_{A_{i}}: D\left(\delta_{A_{i}}\right) \rightarrow L^{2}(M) \bar{\otimes} L^{2}(M)$, where $D\left(\delta_{A_{i}}\right)$ is the algebra generated by $A_{1}, \ldots, A_{n}, \delta_{A_{i}}(a)=a \otimes 1-1 \otimes a$ if $a \in A_{i}$, and $\delta_{A_{i}}(a)=0$ if $a \in A_{j}$ for $j \neq i$. See [Vo99, Section 5] for more details.

Assuming that $\varphi^{*}\left(A_{1} ; \ldots ; A_{n}\right)=\sum_{i=1}^{n}\left\|\delta_{A_{i}}^{*}(1 \otimes 1)\right\|_{2}^{2}<\infty$, Voiculescu proved that $\delta_{A_{i}}$ is a closable derivation [Vo99, Corollary 6.3]. Note that the combination of Remark 9.2 (f), Proposition 5.9 (a) and Definition 9.1 from [Vo99] gives that

$$
\varphi^{*}\left(A_{1} ; W^{*}\left(A_{2}, \ldots, A_{n}\right)\right)=\varphi^{*}\left(A_{1} ; \mathbb{C}\left\langle A_{2}, \ldots, A_{n}\right\rangle\right) \leq 2 \varphi^{*}\left(A_{1} ; \ldots ; A_{n}\right) .
$$

Finally, we will also need the following relation between Fisher information and liberation Fisher information [Vo99, Corollary 5.11]: if $\Phi^{*}\left(X_{1}, \ldots, X_{n}\right)<\infty$, then $\varphi^{*}\left(W^{*}\left(X_{1}\right) ; \ldots ; W^{*}\left(X_{n}\right)\right)<\infty$.

Corollary 4.3. Let $(M, \tau)$ be a tracial von Neumann algebra which is generated by $n \geqslant 2$ non-trivial $(\neq \mathbb{C} 1)$ von Neumann subalgebras $A_{1}, \ldots, A_{n}$. Assume that $A_{1}, \ldots, A_{n}$ have finite liberation Fisher information $\varphi^{*}\left(A_{1} ; \ldots ; A_{n}\right)<\infty, A_{1}$ is diffuse, and $A_{2}$ is a non-amenable $I I_{1}$ factor.

Then $M$ is a non- $L^{2}$-rigid $I I_{1}$ factor. Thus, $M$ is prime, does not have property $\Gamma$ nor property $(T)$. In particular, this is the case if $M$ is generated by $m \geqslant 3$ self-adjoint elements $X_{1}, \ldots, X_{m}$ satisfying $\Phi^{*}\left(X_{1}, \ldots, X_{m}\right)<\infty$.

Proof. Since $A_{1}$ is diffuse, by arguing as in Da10a, Theorem 1] it follows that $M$ is a factor. Indeed, let $x \in \mathcal{Z}(M)$ and define $\eta_{\alpha}: M \rightarrow M$ by $\eta_{\alpha}=\alpha\left(\alpha+\delta_{A_{2}}^{*} \delta_{A_{2}}\right)^{-1}$, for $\alpha>0$. If $y \in A_{1}$, then $\delta_{A_{2}}(y)=0$, hence $\eta_{\alpha}(y)=y$. This implies that $\eta_{\alpha}(y x)=y \eta_{\alpha}(x)$ and $\eta_{\alpha}(x y)=\eta_{\alpha}(x) y$. In particular, we get that $\left[\delta_{A_{2}}\left(\eta_{\alpha}(x)\right), y\right]=$ $\delta_{A_{2}}\left(\eta_{\alpha}([x, y])\right)=0$, for all $\alpha>0$ and $y \in A_{1}$. Since $A_{1}$ is diffuse and $\delta_{A_{2}}\left(\eta_{\alpha}(x)\right)$ can be viewed as a Hilbert-Schmidt operator, we deduce that $\delta_{A_{2}}\left(\eta_{\alpha}(x)\right)=0$. Since $\left\|\eta_{\alpha}(x)-x\right\|_{2} \rightarrow 0$, as $\alpha \rightarrow \infty$, and $\delta_{A_{2}}$ is closable, we get that $x \in D\left(\bar{\delta}_{A_{2}}\right)$ and $\bar{\delta}_{A_{2}}(x)=0$. Thus, for every $y \in A_{2}$, we have $0=\bar{\delta}_{A_{2}}([x, y])=[x, y \otimes 1-1 \otimes y]=$ $[y,[x, 1 \otimes 1]]$. Now, since $A_{2}$ is diffuse we get that $[x, 1 \otimes 1]=0$, which implies that $x \in \mathbb{C} 1$, as claimed.

Since $A_{2}$ is a non-amenable $\mathrm{II}_{1}$ factor, by Co76] we can find a non-amenability set $S \subset A_{2}$. But then $S$ is a non-amenability set for $M$ which is contained in $D\left(\delta_{A_{2}}\right)$. 
Since $A_{1}$ is diffuse, $\delta_{A_{2}}$ is not inner and hence is not bounded by Pe09a, Theorem 2.2]. Thus, we can apply Theorem 1.1 to derive the conclusion.

Now, assume that $M$ is generated by $m \geqslant 3$ self-adjoint elements with $\Phi^{*}\left(X_{1}, \ldots, X_{m}\right)<\infty$. Let $A_{1}$ be the von Neumann algebra generated by $X_{1}$ and $A_{2}$ the von Neumann algebra generated by $X_{2}, \ldots, X_{m}$. Since $\Phi^{*}\left(X_{1}, \ldots, X_{m}\right)<\infty$, as in the proof of Corollary 1.2 , we deduce that the distributions of $X_{1}, \ldots, X_{m}$ have no atoms. As a consequence, $A_{1}$ and $A_{2}$ are diffuse. Moreover, by Da10a, Theorem 13], $A_{2}$ is a non-amenable $\mathrm{II}_{1}$ factor.

Since $\Phi^{*}\left(X_{1}, \ldots, X_{m}\right)<\infty$, we have that $\varphi^{*}\left(W^{*}\left(X_{1}\right) ; \ldots ; W^{*}\left(X_{n}\right)\right)<\infty$. By using equation (4.1) we further get that $\varphi^{*}\left(A_{1} ; A_{2}\right) \leqslant 2 \varphi^{*}\left(W^{*}\left(X_{1}\right) ; \ldots ; W^{*}\left(X_{m}\right)\right)$ $<\infty$. Altogether, the above shows that $M$ is a non- $L^{2}$-rigid factor.

\section{Lipschitz conjugate variables and absence of Cartan subalgebras}

This section is devoted to the proof of Theorem 1.3

5.1. Proof of Theorem 1.3. We claim that the Lipschitz condition implies that the first and second conjugate variables are bounded (i.e. they belong to $M$ ). Firstly, Voiculescu (see equation (1) in [Da10a]) noticed that for all $x \in M_{0}=$ $\mathbb{C}\left\langle X_{1}, \ldots, X_{n}\right\rangle$ we have

$$
\sum_{i=1}^{n}\left(\delta_{i}(x)\left(X_{i} \otimes 1\right)-\left(1 \otimes X_{i}\right) \delta_{i}(x)\right)=x \otimes 1-1 \otimes x .
$$

Moreover, this identity holds for every $x \in D(\bar{\delta})$. Thus, if $x \in D(\bar{\delta})$ satisfies $\bar{\delta}(x) \in\left(M \bar{\otimes} M^{o p}\right)^{\oplus n}$, then $x=\tau(x)+E_{M \otimes 1}\left(\sum_{i=1}^{n}\left(\delta_{i}(x)\left(X_{i} \otimes 1\right)-\left(1 \otimes X_{i}\right) \delta_{i}(x)\right)\right)$ and therefore $x \in M$. This implies that $\mathcal{J}_{1}\left(X_{i}: \mathbb{C}\left\langle X_{1}, \ldots, X_{i-1}, X_{i+1}, \ldots, X_{n}\right\rangle=\right.$ $\xi_{i}=\delta_{i}^{*}(1 \otimes 1) \in M$, for every $i \in\{1, \ldots, n\}$. Now, recall that

$$
\jmath_{2}\left(X_{i}: \mathbb{C}\left\langle X_{1}, \ldots, X_{i-1}, X_{i+1}, \ldots, X_{n}\right\rangle\right)=\delta_{i}^{*}\left(\xi_{i} \otimes 1\right) .
$$

Since by the second formula in Vo98b, Proposition 4.1] (applied to $a=\xi_{i}$ and $\eta=1 \otimes 1)$ we have that $\delta_{i}^{*}\left(\xi_{i} \otimes 1\right)=\xi_{i}^{2}-(\tau \otimes \mathrm{id})\left(\bar{\delta}_{i}\left(\xi_{i}\right)\right)$, we conclude that $\mathcal{J}_{2}\left(X_{i}: \mathbb{C}\left\langle X_{1}, \ldots, X_{i-1}, X_{i+1}, \ldots, X_{n}\right\rangle\right) \in M$, for all $i \in\{1, \ldots, n\}$. This proves our claim.

Since $\Phi^{*}\left(X_{1}, \ldots, X_{n}\right)=\sum_{i=1}^{n}\left\|\delta_{i}^{*}(1 \otimes 1)\right\|_{2}^{2}<\infty$, Da10a, Theorem 13] implies that $M$ is a $\mathrm{II}_{1}$ factor without property Gamma. Moreover, since the first and second order conjugate variables are bounded, $S=\left\{X_{1}, \ldots, X_{n}\right\}$ is a non-Gamma set for $M$ (see [Da10a, Lemmas 9 and 10, and Remark 11]). Lemma 2.10 gives that $S$ is a non-amenability set for $M$.

Let $Q$ be either $\mathbb{C} 1$ or a $\mathrm{II}_{1}$ factor. Our aim is to show that $M \bar{\otimes} Q$ does not have a Cartan subalgebra. Assume by contradiction that there is a Cartan subalgebra $A \subset M \bar{\otimes} Q$. Denote $\tilde{M}=M * L\left(\mathbb{F}_{\infty}\right)$. Let $\alpha_{t}: M \rightarrow \tilde{M}$ be the $*$-homomorphisms provided by Theorem 3.6. We extend $\alpha_{t}$ to a $*$-homomorphism $\alpha_{t}: M \bar{\otimes} Q \rightarrow \tilde{M} \bar{\otimes} Q$ by letting $\alpha_{t \mid Q}=\mathrm{id}_{Q}$.

The rest of the proof is divided between three claims.

Claim 1. Given $t \geqslant 0$, there exist projections $p_{t}, q_{t}, r_{t} \in \mathcal{Z}\left(\alpha_{t}(M)^{\prime} \cap \tilde{M}\right)$ satisfying $p_{t}+q_{t}+r_{t}=1$ :

(1) $u_{t} \alpha_{t}(M) p_{t} u_{t}^{*} \subset M$, for some unitary $u_{t} \in \tilde{M}$,

(2) $v_{t} \alpha_{t}(M) q_{t} v_{t}^{*} \subset L\left(\mathbb{F}_{\infty}\right)$, for some unitary $v_{t} \in \tilde{M}$,

(3) $\alpha_{t}(M) r_{t} \nprec_{\tilde{M}} M$ and $\alpha_{t}(M) r_{t} \nprec_{\tilde{M}} L\left(\mathbb{F}_{\infty}\right)$. 
Proof of Claim 1. Since $M$ and $L\left(\mathbb{F}_{\infty}\right)$ are factors, the sets of projections satisfying (1) and (2) are closed taking supremum. Let $p_{t}, q_{t} \in \mathcal{Z}\left(\alpha_{t}(M)^{\prime} \cap \tilde{M}\right)$ be the maximal projections satisfying (1) and (2). [IPP08, Theorem 1.2.1] implies that $p_{t} q_{t}=0$. Let $r_{t}=1-p_{t}-q_{t}$. If $\alpha_{t}(M) r_{t} \prec_{\tilde{M}} M$, then, as $M$ is a factor, the proof of [IPP08, Theorem 5.1] yields a non-zero projection $r \in \mathcal{Z}\left(\alpha_{t}(M)^{\prime} \cap \tilde{M}\right) r_{t}$ and a unitary $u \in \tilde{M}$ such that $u \alpha_{t}(M) r u^{*} \subset M$. Since $r \leqslant r_{t}$, this contradicts the maximality of $p_{t}$. Similarly, $\alpha_{t}(M) r_{t} \prec_{\tilde{M}} L\left(\mathbb{F}_{\infty}\right)$ contradicts the maximality of $q_{t}$.

Claim 2. $r_{t}=0$, for all $t \geqslant 0$.

Proof of Claim 2. Suppose by contradiction that $r_{t} \neq 0$, for some $t \geqslant 0$.

Since $M$ is a non-amenable $\mathrm{II}_{1}$ factor, by using (3) from Claim 1 and applying [o15, Theorem 6.3] to the inclusion $\alpha_{t}(M) r_{t} \subset \tilde{M}=M * L\left(\mathbb{F}_{\infty}\right)$ we get that $\left(\alpha_{t}(M) r_{t}\right)^{\prime} \cap\left(r_{t} M r_{t}\right)^{\omega} \prec_{\tilde{M}^{\omega}} \mathbb{C} 1$. Thus, there exists a non-zero projection $r_{t}^{\prime} \in$ $\mathcal{Z}\left(\left(\alpha_{t}(M) r_{t}\right)^{\prime} \cap\left(r_{t} \tilde{M} r_{t}\right)^{\omega}\right)=\mathcal{Z}\left(\alpha_{t}(M)^{\prime} \cap \tilde{M}^{\omega}\right) r_{t}$ such that $r_{t}^{\prime}\left(\alpha_{t}(M)^{\prime} \cap \tilde{M}^{\omega}\right) r_{t}^{\prime}$ is completely atomic. By [o15, Lemma 2.7] it follows that $r_{t}^{\prime} \in \tilde{M}$ and moreover $r_{t}^{\prime}\left(\alpha_{t}(M)^{\prime} \cap \tilde{M}^{\omega}\right) r_{t}^{\prime}=r_{t}^{\prime}\left(\alpha_{t}(M)^{\prime} \cap \tilde{M}\right) r_{t}^{\prime}$.

By combining these facts we deduce that there exists a non-zero projection $r_{t}^{\prime \prime} \in$ $\alpha_{t}(M)^{\prime} \cap \tilde{M}$ such that $r_{t}^{\prime \prime} \leqslant r_{t}^{\prime}$ and $r_{t}^{\prime \prime}\left(\alpha_{t}(M)^{\prime} \cap \tilde{M}^{\omega}\right) r_{t}^{\prime \prime}=\mathbb{C} r_{t}^{\prime \prime}$. Thus, we have $\left(\alpha_{t}(M) r_{t}^{\prime \prime}\right)^{\prime} \cap\left(r_{t}^{\prime \prime} \tilde{M} r_{t}^{\prime \prime}\right)^{\omega}=\mathbb{C} r_{t}^{\prime \prime}$.

Next, we denote still by $r_{t}^{\prime \prime}$ the projection $r_{t}^{\prime \prime} \otimes 1 \in \tilde{M} \bar{\otimes} Q$. We define $A_{1}=$ $\alpha_{t}(A) r_{t}^{\prime \prime}$ and note that $P_{1}:=\mathcal{N}_{r_{t}^{\prime \prime}(M \bar{\otimes} Q) r_{t}^{\prime \prime}}\left(A_{1}\right)^{\prime \prime}$ contains $\left(\alpha_{t}(M) \bar{\otimes} Q\right) r_{t}^{\prime \prime}$. In particular, $P_{1}$ contains $P_{0}:=\alpha_{t}(M) r_{t}^{\prime \prime} \otimes 1$. Since $\left(\alpha_{t}(M) r_{t}^{\prime \prime}\right)^{\prime} \cap\left(r_{t}^{\prime \prime} \tilde{M} r_{t}^{\prime \prime}\right)^{\omega}=\mathbb{C} r_{t}^{\prime \prime}$, by applying Theorem 2.12 we get that one of the following conditions holds: (a) $A_{1} \prec_{\tilde{M} \bar{\otimes} Q} 1 \otimes Q$, (b) $P_{1} \prec_{\tilde{M}} M$ or $P_{1} \prec_{\tilde{M}} L\left(\mathbb{F}_{\infty}\right)$, or (c) $P_{0}$ is amenable.

Now, it is easy to see that (a) implies that $A \prec_{M \bar{\otimes} Q} 1 \otimes Q$. By taking relative commutants (see e.g. VVa08, Lemma 3.5]) it follows that $M \otimes 1 \prec_{M \otimes \bar{Q}} A$. Since $M$ is non-amenable while $A$ is abelian, this is a contradiction. By using (3) from Claim 1 and the fact that $M$ is non-amenable, we get that (b) and (c) cannot hold either. This altogether provides the desired contradiction.

Claim 3. $\tau\left(q_{t}\right) \rightarrow 0$, as $t \rightarrow 0$.

Proof of Claim 3. Since the $M-M$ bimodule $L^{2}\left(\left\langle\tilde{M}, e_{L\left(\mathbb{F}_{\infty}\right)}\right\rangle\right)$ is isomorphic to $\left(L^{2}(M) \bar{\otimes} L^{2}(M)\right)^{\oplus \infty}$ and $M$ is non-amenable, we get that $M$ is not amenable relative to $L\left(\mathbb{F}_{\infty}\right)$ inside $\tilde{M}$. Since $M$ is a factor, we also have that $M^{\prime} \cap \tilde{M}=\mathbb{C} 1$ (see [Po83, Remark 6.3]). By [OP10, Corollary 2.3] we derive that for any net of vectors $\xi_{n} \in L^{2}\left(\left\langle\tilde{M}, e_{L\left(\mathbb{F}_{\infty}\right)}\right\rangle\right)$ satisfying $\left\|x \xi_{n}\right\|_{2} \leqslant\|x\|_{2}$, for all $x \in \tilde{M}$, and $\left\|y \xi_{n}-\xi_{n} y\right\|_{2} \rightarrow 0$, for all $y \in M$, we must have that $\left\|\xi_{n}\right\|_{2} \rightarrow 0$.

Let $\xi_{t}=v_{t}^{*} e_{L\left(\mathbb{F}_{\infty}\right)} v_{t} q_{t} \in L^{2}\left(\left\langle\tilde{M}, e_{L\left(\mathbb{F}_{\infty}\right)}\right\rangle\right)$, for any $t \geqslant 0$. Since $v_{t} \alpha_{t}(M) q_{t} v_{t}^{*} \subset$ $L\left(\mathbb{F}_{\infty}\right)$, we get that $\alpha_{t}(y) \xi_{t}=\xi_{t} \alpha_{t}(y)$, for all $y \in M$. Also, $\left\|x \xi_{t}\right\|_{2},\left\|\xi_{t} x\right\|_{2} \leqslant\|x\|_{2}$, for all $x \in \tilde{M}$ and every $t \geqslant 0$. Thus, if $y \in M$, then $\left\|y \xi_{t}-\xi_{t} y\right\|_{2} \leqslant 2\left\|y-\alpha_{t}(y)\right\|_{2}$. Since $\left\|\alpha_{t}(y)-y\right\|_{2} \rightarrow 0$, by using the previous paragraph we conclude that $\left\|\xi_{t}\right\|_{2} \rightarrow$ 0 , as $t \rightarrow 0$. Since $\left\|\xi_{t}\right\|_{2}^{2}=\tau\left(q_{t}\right)$, we are done.

We are now ready to derive a contradiction. Recall that $S$ is a non-amenability set for $M$ and that the $M-M$ bimodule $L^{2}(\tilde{M}) \ominus L^{2}(M)$ is isomorphic to $\left(L^{2}(M) \bar{\otimes} L^{2}(M)\right)^{\oplus \infty}$. Since $u_{t} \alpha_{t}(M) p_{t} u_{t}^{*} \subset M$, Lemma 2.7 (2) implies that we 
can find $C>0$ such that for all $t \geqslant 0$ we have

$$
\left\|\left(\alpha_{t}(x)-E_{M}\left(\alpha_{t}(x)\right)\right) p_{t}\right\|_{2} \leqslant C \sum_{y \in S}\left\|\left(\alpha_{t}(y)-y\right) p_{t}\right\|_{2}, \text { for all } x \in(M)_{1} .
$$

Now, let $M_{0}$ be the $*$-algebra generated by $\left\{X_{1}, \ldots, X_{n}\right\}$ and fix $x \in M_{0}$ with $\|x\| \leqslant 1$. Theorem 3.6 gives that $\left\|\frac{1}{\sqrt{t}}\left(\alpha_{t}(x)-x\right)-\sum_{i=1}^{n} \delta_{i}(x) \# S_{i}^{(t)}\right\|_{2} \rightarrow 0$. Note also that $E_{M}\left(\delta_{i}(x) \# S_{i}^{(t)}\right)=0$ and $\left\|\sum_{i=1}^{n} \delta_{i}(x) \# S_{i}^{(t)}\right\|_{2}=\sqrt{\sum_{i=1}^{n}\left\|\delta_{i}(x)\right\|_{2}^{2}}=\|\delta(x)\|_{2}$. Claims 2 and 3 together imply that $\left\|p_{t}-1\right\|_{2} \rightarrow 0$. By combining all of these facts and (5.2) we deduce that $\|\delta(x)\|_{2} \leqslant C \sum_{y \in S}\|\delta(y)\|_{2}$. Since $x \in\left(M_{0}\right)_{1}$ is arbitrary, we deduce that $\delta$ is bounded. As in the proof of Corollary 1.2, this leads to a contradiction.

\section{Primeness and absence of Cartan subalgebras FOR REGULARIZED ALGEBRAS}

In this section we establish indecomposibility results for algebras obtained by free additive convolution and liberation. Firstly, for free additive convolution by semicircular variables $\left\{S_{1}, \ldots, S_{n}\right\}$, we prove that algebras of the form $M_{\varepsilon}=$ $\left\{X_{1}+\varepsilon S_{1}, \ldots, X_{n}+\varepsilon S_{n}\right\}^{\prime \prime}$ are prime and do not have Cartan subalgebras. More precisely, we have

Theorem 6.1. Let $(M, \tau)$ be a tracial von Neumann algebra and $X_{1}, \ldots, X_{n} \in$ $M$ be $n \geqslant 2$ self-adjoint elements. Let $\left\{S_{1}, \ldots, S_{n}\right\} \in L\left(\mathbb{F}_{n}\right)$ be the canonical semicircular family and $\varepsilon>0$. Denote by $M_{\varepsilon} \subset M * L\left(\mathbb{F}_{n}\right)$ the von Neumann subalgebra generated by $X_{1}+\varepsilon S_{1}, \ldots, X_{n}+\varepsilon S_{n}$.

Then $M_{\varepsilon}$ is a non- $L^{2}$-rigid $I I_{1}$ factor that does not have a Cartan subalgebra.

Proof. Fix $\varepsilon>0$. Then we have that $\mathcal{J}_{p}\left(X_{i}^{\varepsilon}: \mathbb{C}\left\langle X_{1}^{\varepsilon}, \ldots, X_{i-1}^{\varepsilon}, X_{i+1}^{\varepsilon}, \ldots, X_{n}^{\varepsilon}\right\rangle\right)$ exists and belongs to $M_{\varepsilon}$, for all $p \in\{1,2\}$ and $i \in\{1, \ldots, n\}$, by Vo98b, Corollary 3.9]. By Corollary 1.2 it follows that $M_{\varepsilon}$ is a non- $L^{2}$-rigid $\mathrm{II}_{1}$ factor.

Now, assume by contradiction that $M_{\varepsilon}$ has a Cartan subalgebra and denote $\tilde{M}=M * L\left(\mathbb{F}_{n}\right)$. After enlarging $M$ if necessary (e.g. by replacing it with $M * L\left(\mathbb{F}_{2}\right)$ ) we may assume that it is a factor. Since $M_{\varepsilon}$ is a non-amenable factor and $M, L\left(\mathbb{F}_{n}\right)$ are factors, by [Io15, Corollary 9.1] we can find unitary elements $u, v \in \tilde{M}$ and projections $p, q \in \mathcal{Z}\left(M_{\varepsilon}^{\prime} \cap \tilde{M}\right)$ such that $u M_{\varepsilon} p u^{*} \subset M, v M_{\varepsilon} q v^{*} \subset L\left(\mathbb{F}_{n}\right)$ and $p+q=1$. Since $L\left(\mathbb{F}_{n}\right)$ is strongly solid [OP10, we must have that $q=0$, hence $u M_{\varepsilon} u^{*} \subset M$.

Towards a contradiction, let $i \in\{1, . ., n\}$. We define $A_{i}, M_{i}$ and $N_{i}$ to be the von Neumann subalgebras of $\tilde{M}$ generated by $\left\{X_{i}+\varepsilon S_{i}\right\}, M \cup\left\{S_{i}\right\}$ and $\left\{S_{j}\right\}_{j \in\{1, \ldots, n\} \backslash\{i\}}$, respectively. Following [Vo93, Proposition 4.7], the distribution of $X_{i}+\varepsilon S_{i}$ is absolutely continuous with respect to the Lebesgue measure on $\mathbb{R}$; hence it has no atoms. This implies that $A_{i}$ is diffuse.

Since $u A_{i} u^{*} \subset M \subset M_{i}, A_{i} \subset M_{i}$ and $\tilde{M}=M_{i} * N_{i}$, by applying Po83 or [IPP08, Theorem 1.2.1] we derive that $u \in M_{i}$. Since $i \in\{1, \ldots, n\}$ was arbitrary, we conclude that $u \in \bigcap_{i=1}^{n} M_{i}$. Now, freeness easily yields that $\bigcap_{i=1}^{n} M_{i}=M$ and therefore $u \in M$. Thus, we would get that $M_{\varepsilon} \subset M$. This would imply that $S_{i} \in M$, for all $i \in\{1, \ldots, n\}$, thus giving a contradiction.

Our techniques allow us to more generally handle the case of regularization by variables $\left\{Y_{1}, \ldots, Y_{n}\right\}$ that have bounded first and second order conjugate variables. 
Theorem 6.2. Let $\left(M_{1}, \tau_{1}\right),\left(M_{2}, \tau_{2}\right)$ be tracial von Neumann algebras and let $M=M_{1} * M_{2}$. Let $X_{1} \in M_{1} \backslash \mathbb{C} 1, X_{2}, \ldots, X_{n} \in M_{1}$ and $Y_{1}, \ldots, Y_{n} \in M_{2} \backslash \mathbb{C} 1$ be self-adjoint elements, for some $n \geqslant 2$. Denote by $N \subset M$ the von Neumann subalgebra generated by $Z_{1}=X_{1}+Y_{1}, \ldots, Z_{n}=X_{n}+Y_{n}$.

Assume that $Y_{1}, \ldots, Y_{n}$ have bounded first and second order conjugate variables; i.e. we have that $\mathcal{J}_{p}\left(Y_{i}: \mathbb{C}\left\langle Y_{1}, \ldots, Y_{i-1}, Y_{i+1}, \ldots, Y_{n}\right\rangle\right)$ exists and belongs to $M_{2}$, for all $p \in\{1,2\}$ and $i \in\{1, \ldots, n\}$.

Then $N$ is a non- $L^{2}$-rigid $I I_{1}$ factor that does not have a Cartan subalgebra.

Proof. Without loss of generality, we may assume that $M_{1}$ and $M_{2}$ are factors and that $\tau\left(Y_{i}\right)=0$, for all $i \in\{1, \ldots, n\}$. Let $M_{0} \subset M$ be the $*$-subalgebra generated by $M_{1}$ and $\left\{Y_{1}, \ldots, Y_{n}\right\}$. We define two real closable derivations $\delta_{1}, \delta_{2}: M_{0} \rightarrow$ $L^{2}(M) \bar{\otimes} L^{2}(M)$, by letting $\delta_{1}(x)=0$ and $\delta_{2}(x)=i[x, 1 \otimes 1]$, if $x \in M_{1}$, and $\delta_{1}(y)=i[y, 1 \otimes 1]$ and $\delta_{2}(y)=0$, if $y \in M_{2}$.

We continue by proving three facts.

Firstly, let $i \in\{1, \ldots, n\}$. Since $Y_{1}, \ldots, Y_{n}$ have bounded first and second order conjugate variables, $Y_{i}$ has finite free entropy. By [Vo94, Proposition 4.5], the distribution of $Y_{i}$ is absolutely continuous with respect to the Lebesgue measure on $\mathbb{R}$. In particular, the distribution of $Y_{i}$ has no atoms.

Secondly, by [Vo98b, Proposition 3.7] we have that the conjugate variables

$\mathcal{J}_{p}\left(Z_{i}: \mathbb{C}\left\langle Z_{1}, \ldots, Z_{i-1}, Z_{i+1}, \ldots, Z_{n}\right\rangle\right)=E_{N}\left(\mathcal{J}_{p}\left(Y_{i}: \mathbb{C}\left\langle Y_{1}, \ldots, Y_{i-1}, Y_{i+1}, \ldots, Y_{n}\right\rangle\right)\right)$

exist and belong to $N$, for every $p \in\{1,2\}, i \in\{1, \ldots, n\}$. By combining Da10a, Remark 11] and Lemma 2.10 we get that $F=\left\{Z_{1}, \ldots, Z_{n}\right\}$ is a non-Gamma, hence a non-amenability, set for $N$.

Thirdly, let us prove that $\bigcap_{i=1}^{n} L^{2}\left(\left\{M_{1}, Y_{i}\right\}^{\prime \prime}\right)=L^{2}\left(M_{1}\right)$. We start by considering the free difference quotient $\partial_{i}: M_{0} \rightarrow L^{2}(M) \bar{\otimes} L^{2}(M)$ with respect to $Y_{i}$ given by $\partial_{i}(x)=0$, for $x \in M_{1}$, and $\partial_{i}\left(Y_{j}\right)=\delta_{i, j} 1 \otimes 1$, for $j \in\{1, \ldots, n\}$. By Vo98b, Proposition 3.6], $\partial_{i}^{*}(1 \otimes 1)$ exists and is equal to $\mathcal{J}_{1}\left(Y_{i}: \mathbb{C}\left\langle Y_{1}, \ldots, Y_{i-1}, Y_{i+1}, \ldots, Y_{n}\right\rangle\right)$. By [Vo98b, Corollary 4.2] we deduce that $M_{0} \otimes M_{0} \subset D\left(\partial_{i}^{*}\right)$.

Now, let $Z \in \bigcap_{i=1}^{n} L^{2}\left(\left\{M_{1}, Y_{i}\right\}^{\prime \prime}\right)$. Towards proving that $Z \in L^{2}\left(M_{1}\right)$, take a sequence $Z_{n} \in M_{0}$ such that $\left\|Z_{n}-Z\right\|_{2} \rightarrow 0$. If $i \in\{1, \ldots, n\}$, then $Z \in D\left(\bar{\partial}_{i}\right)$ and $\bar{\partial}_{i}(Z)=0$. Since $M_{0} \otimes M_{0} \subset D\left(\partial_{i}^{*}\right)$ we deduce that $\left\langle\partial_{i}\left(Z_{n}\right), \zeta\right\rangle \rightarrow 0$, for all $\zeta \in M_{0} \otimes M_{0}$. Also, a variant of an identity due to Voiculescu (see Da10a, equation (1)]) gives that $\delta_{1}\left(Z_{n}\right)=i \sum_{j=1}^{n}\left[\partial_{j}\left(Z_{n}\right)\left(X_{j} \otimes 1\right)-\left(1 \otimes X_{j}\right) \partial_{j}\left(Z_{n}\right)\right]$.

We therefore conclude that $\left\langle\delta_{1}\left(Z_{n}\right), \zeta\right\rangle \rightarrow 0$, for all $\zeta \in M_{0} \otimes M_{0}$. This implies that $Z \in D\left(\bar{\delta}_{1}\right)$ and $\bar{\delta}_{1}(Z)=0$. From this it is easy to see that $Z \in L^{2}\left(M_{1}\right)$.

Suppose that $N$ either has a Cartan subalgebra or is $L^{2}$-rigid. Towards getting a contradiction, we first use the fact that $F$ is contained in the domains of $\delta_{1}$ and $\delta_{2}$ to prove the following:

Claim 1. There exists $\xi \in L^{2}(M) \bar{\otimes} L^{2}(M)$ such that $\delta_{1}(x)=[x, \xi]$, for all $x \in$ $N \cap M_{0}$.

Proof of Claim 1. Let $\tilde{M}=M * L(\mathbb{Z})$ and $s \in L(\mathbb{Z})$ be a generating $(0,1)$ semicircular element. For $t \in \mathbb{R}$ and $j \in\{1,2\}$, we define $\alpha_{t}^{(j)} \in \operatorname{Aut}(\tilde{M})$ by letting $\alpha_{t}^{(j)}(x)=x$ if $x \in M_{j}$, and $\alpha_{t}^{(j)}(y)=\exp (i t s) y \exp (-i t s)$ if $y \in M_{k} * L(\mathbb{Z})$, where $k$ is such that $\{j, k\}=\{1,2\}$. Then it is easy to see (e.g. by combining Proposition 
3.3 and Remark 3.4 that

$$
\left\|\frac{1}{t}\left(\alpha_{t}^{(j)}(x)-x\right)-\delta_{j}(x) \# s\right\|_{2} \rightarrow 0, \quad \text { as } t \rightarrow 0, \text { for all } x \in M_{0} .
$$

To get the conclusion, we treat separately the two cases. Assume first that $N$ has a Cartan subalgebra. Since $N$ is a non-amenable factor and $M_{1}, M_{2}$ are factors, [Io15, Corollary 9.1] implies that we can find unitary elements $u_{1}, u_{2} \in M$ and projections $p_{1}, p_{2} \in \mathcal{Z}\left(N^{\prime} \cap M\right)$ such that $u_{1} N p_{1} u_{1}^{*} \subset M_{1}, u_{2} N p_{2} u_{2}^{*} \subset M_{2}$ and $p_{1}+p_{2}=1$.

Let $t \in \mathbb{R}$ and $i \in\{1,2\}$. Then $\alpha_{t}^{(i)}\left(u_{i}\right) \alpha_{t}^{(i)}(N) \alpha_{t}^{(i)}\left(p_{i}\right) \alpha_{t}^{(i)}\left(u_{i}\right)^{*} \subset \alpha_{t}^{(i)}\left(M_{i}\right) \subset M$. Also, the $M-M$ bimodule $L^{2}(\tilde{M}) \ominus L^{2}(M)$ is isomorphic to $\left(L^{2}(M) \bar{\otimes} L^{2}(M)\right)^{\oplus \infty}$. Since $F$ is a non-amenability set for $N$, Lemma 2.7 (2) provides a constant $C>0$ such that for all $x \in(N)_{1}$ we have

$$
\left\|\left(\alpha_{t}^{(i)}(x)-E_{M}\left(\alpha_{t}^{(i)}(x)\right)\right) \alpha_{t}^{(i)}\left(p_{i}\right)\right\|_{2} \leqslant C \sum_{y \in F}\left\|\alpha_{t}^{(i)}(y)-y\right\|_{2} .
$$

Note that $E_{M}\left(\delta_{i}(x) \# s\right)=0$, for all $x \in M_{0}$. Thus, combining equations (6.1) and (6.2) yields that $\left\|\delta_{i}(x) p_{i}\right\|_{2} \leqslant C \sum_{y \in F}\|\delta(y)\|_{2}$, for all $x \in\left(N \cap M_{0}\right)_{1}$. Since $\delta_{1}(x) p_{2}=i[x, 1 \otimes 1] p_{2}-\delta_{2}(x) p_{2}$, we get that $\left\|\delta_{1}(x) p_{2}\right\|_{2} \leqslant 2+C \sum_{y \in F}\|\delta(y)\|_{2}$, for all $x \in\left(N \cap M_{0}\right)_{1}$. Since $p_{1}+p_{2}=1$, it follows that the restriction of $\delta_{1}$ to $N \cap M_{0}$ is bounded. The conclusion follows as in the proof of [Pe09a, Theorem 2.2].

Now, assume that $N$ is $L^{2}$ rigid. Note that the restriction of $\delta_{1}$ to $N \cap M_{0}$ is a real closable derivation into $L^{2}(M) \bar{\otimes} L^{2}(M)$. Since $L^{2}(M) \bar{\otimes} L^{2}(M)$ is isomorphic to an $N-N$ sub-bimodule of $\left(L^{2}(N) \bar{\otimes} L^{2}(N)\right)^{\oplus \infty}$ and $N \cap M_{0}$ contains a non-amenability set for $N$, Theorem 1.1 implies that $\delta_{1}$ is bounded on $N \cap M_{0}$. The conclusion now follows as above.

Now, by the definition of $\delta_{1}$ we have that $\delta_{1}\left(Z_{j}\right)=i\left[Y_{j}, 1 \otimes 1\right]$. Denote $\eta=-i \xi \in$ $L^{2}(M) \bar{\otimes} L^{2}(M)$. Since Claim 1 yields that $\delta_{1}\left(Z_{i}\right)=\left[Z_{i}, \xi\right]$, we conclude that

$$
\left[Y_{i}, 1 \otimes 1\right]=\left[Z_{i}, \eta\right], \text { for all } i \in\{1, \ldots, n\} .
$$

Next, we identify $L^{2}(M) \bar{\otimes} L^{2}(M)$ with $L^{2}\left(M \bar{\otimes} M^{o p}\right)$ in the natural way such that the $M-M$ bimodule structure of $L^{2}(M) \bar{\otimes} L^{2}(M)$ corresponds to the left multiplication action of $M \bar{\otimes} M^{o p}$ on $L^{2}\left(M \bar{\otimes} M^{o p}\right)$. We can therefore rewrite (6.3) as

$$
Y_{i} \otimes 1-1 \otimes Y_{i}^{o p}=\left(Z_{i} \otimes 1-1 \otimes\left(Z_{i}\right)^{o p}\right) \eta, \text { for all } i \in\{1, \ldots, n\} .
$$

Claim 2. $\eta \in L^{2}\left(M_{1}\right) \bar{\otimes} L^{2}\left(M_{1}\right) \cong L^{2}\left(M_{1} \bar{\otimes} M_{1}^{o p}\right)$.

Proof of Claim 2. Fix $i \in\{1, \ldots, n\}$ and denote by $M^{(i)}=\left\{M_{1}, Y_{i}\right\}^{\prime \prime}$ the von Neumann algebra generated by $M_{1}$ and $Y_{i}$. Since $X_{i} \in M_{1} \backslash \mathbb{C} 1$ and the distribution of $Y_{i}$ has no atoms, employing [Be08, Theorem 4.1] we derive that the distribution of $Z_{i}=X_{i}+Y_{i}$ is absolutely continuous with respect to the Lebesgue measure on $\mathbb{R}$ and thus has no atoms. This implies that the self-adjoint element $T_{i}:=$ $Z_{i} \otimes 1-1 \otimes\left(Z_{i}\right)^{o p} \in M \bar{\otimes} M^{o p}$ has no kernel.

For $\delta>0$, let $A_{\delta}=\{r \in \mathbb{R}|| r \mid \geqslant \delta\}$ and $f_{\delta}: \mathbb{R} \rightarrow \mathbb{R}$ be the Borel function $f_{\delta}(r)=\frac{1}{r} 1_{A_{\delta}}(r)$. Since $T_{i}$ has no kernel, $f_{\delta}\left(T_{i}\right) T_{i}=1_{A_{\delta}}\left(T_{i}\right) \rightarrow \mathrm{id}$, in the strong operator topology, as $\delta \rightarrow 0$. Thus, by using formula (6.4) we derive that

$$
\left\|f_{\delta}\left(T_{i}\right)\left(Y_{i} \otimes 1-1 \otimes Y_{i}^{o p}\right)-\eta\right\|_{2}=\left\|\left(f_{\delta}\left(T_{i}\right) T_{i}-1\right) \eta\right\|_{2} \rightarrow 0, \quad \text { as } \delta \rightarrow 0 .
$$

Since $Y_{i} \in M^{(i)}$ and $T_{i} \in M^{(i)} \bar{\otimes} M^{(i) o p}$, we get that $f_{\delta}\left(T_{i}\right)\left(Y_{i} \otimes 1-1 \otimes Y_{i}^{o p}\right) \in$ $M^{(i)} \bar{\otimes} M^{(i) o p}$. 
We deduce that $\eta \in L^{2}\left(M^{(i)} \bar{\otimes} M^{(i) o p}\right) \cong L^{2}\left(M^{(i)}\right) \bar{\otimes} L^{2}\left(M^{(i)}\right)$, for all $i \in\{1, \ldots, n\}$. Since we proved that $\bigcap_{i=1}^{n} L^{2}\left(M^{(i)}\right)=L^{2}\left(M_{1}\right)$, we conclude that $\eta \in L^{2}\left(M_{1}\right) \bar{\otimes}$ $L^{2}\left(M_{1}\right)$, as claimed.

Let $P$ be the orthogonal projection from $L^{2}(M) \bar{\otimes} L^{2}(M)$ onto $L^{2}(M) \bar{\otimes} L^{2}\left(M_{1}\right)$. Equation (6.3) gives that $Y_{1} \otimes 1-1 \otimes Y_{1}=Z_{1} \eta-\eta Z_{1}$. Since $E_{M_{1}}\left(Y_{1}\right)=0$ and $\eta \in L^{2}\left(M_{1}\right) \bar{\otimes} L^{2}\left(M_{1}\right)$, by applying $P$ to the last identity, we deduce that $Y_{1} \otimes 1=Z_{1} \eta-\eta X_{1}$. Hence $1 \otimes Y_{1}=\eta Y_{1}$. Since $Y_{1}$ is diffuse, this implies that $\eta=1 \otimes 1$ and further that $X_{1} \otimes 1=1 \otimes X_{1}$. Thus, $X_{1} \in \mathbb{C} 1$, which is the desired contradiction.

Finally, we prove an indecomposability result for regularized algebras obtained by liberation in the sense of [Vo99, Section 2].

Theorem 6.3. Let $\left(M_{1}, \tau_{1}\right),\left(M_{2}, \tau_{2}\right)$ be tracial von Neumann algebras and $M=$ $M_{1} * M_{2}$. Let $A_{1}, \ldots, A_{n} \subset M_{1}$ be diffuse von Neumann subalgebras and $u_{1}, \ldots, u_{n} \in$ $M_{2}$ be unitary elements, for some $n \geqslant 2$. Denote by $N \subset M$ the von Neumann subalgebra generated by $u_{1} A_{1} u_{1}^{*}, \ldots, u_{n} A_{n} u_{n}^{*}$.

Assume that $A_{1}$ is a non-amenable $I I_{1}$ factor and that $u_{2} \notin \mathbb{C} u_{1}$.

Then $N$ is a non- $L^{2}$-rigid $I I_{1}$ factor that does not have a Cartan subalgebra.

Proof. Let us first show that $N$ is a factor. Let $x \in \mathcal{Z}(N)$. Then $\left[u_{1}^{*} x u_{1}, A_{1}\right]=$ $\left[u_{2}^{*} x u_{2}, A_{2}\right]=0$. Since $A_{1}, A_{2} \subset M_{1}$ are diffuse, applying [Po83] or [PP08, Theorem 1.2.1] gives that $u_{1}^{*} x u_{1}$ and $u_{2}^{*} x u_{2}$ belong to $M_{1}$. Equivalently, $x \in u_{1} M_{1} u_{1}^{*} \cap$ $u_{2} M_{1} u_{2}^{*}$. Since $u_{1}, u_{2} \in M_{2}$ and $u_{2} \notin \mathbb{C} u_{1}$, we get that $x \in \mathbb{C} 1$.

Next, let $M_{0} \subset M$ be the *-subalgebra generated by $M_{1}$ and $M_{2}$. Consider the real closable derivation $\delta_{1}: M_{0} \rightarrow L^{2}(M) \bar{\otimes} L^{2}(M)$ by letting $\delta_{1}(x)=0$ if $x \in M_{1}$, and $\delta_{1}(y)=i[y, 1 \otimes 1]$ if $y \in M_{2}$. Since $A_{1}$ is a non-amenable $\mathrm{II}_{1}$ factor, Co76] implies that there exists a non-amenability set $F \subset A_{1}$. Thus, $u_{1} F u_{1}^{*}$ is a non-amenability set for $N$ which is contained in the domain of $\delta_{1}$.

If $N$ is either $L^{2}$-rigid or has a Cartan subalgebra, then the proof of Theorem 6.2 implies that there exists $\xi \in L^{2}(M) \bar{\otimes} L^{2}(M)$ such that $\delta_{1}(x)=[x, \xi]$, for all $x \in N \cap M_{0}$.

To get a contradiction, let $j \in\{1, \ldots, n\}$. Since $A_{j}$ is diffuse, we can find a self-adjoint element $X_{j} \in A_{j}$ which generates a diffuse algebra. If we define $Z_{j}=u_{j} X_{j} u_{j}^{*}$, then we have

$\left[Z_{j}, \xi\right]=\delta_{1}\left(u_{j} X_{j} u_{j}^{*}\right)=i\left(\left[u_{j}, 1 \otimes 1\right] X_{j} u_{j}^{*}+u_{j} X_{j}\left[u_{j}^{*}, 1 \otimes 1\right]\right)=i\left[Z_{j}, 1 \otimes 1-u_{j} \otimes u_{j}^{*}\right]$.

Since $Z_{j}$ is diffuse, we deduce that $\xi=i\left(1 \otimes 1-u_{j} \otimes u_{j}^{*}\right)$ for all $j \in\{1, \ldots, n\}$. This implies in particular that $u_{1} \otimes u_{1}^{*}=u_{2} \otimes u_{2}^{*}$, and hence that $u_{2} \in \mathbb{C} u_{1}$, which gives a contradiction.

\section{Algebraic derivations and absence of Cartan subalgebras}

The main goal of this section is to establish a general result showing absence of Cartan subalgebras for any $\mathrm{II}_{1}$ factor $M$ admitting certain unbounded "algebraic" derivations. More precisely:

Theorem 7.1. Let $M$ be a $I I_{1}$ factor, $B \subset M$ be a von Neumann subalgebra and $M_{0} \subset M$ be a weakly dense *-subalgebra such that $M_{0}$ contains a non-amenability set for $M$ relative to $B$. Assume that for any non-zero projection $r \in B^{\prime} \cap M$, 
there exists a mixing $B-B$ sub-bimodule $\mathcal{H}$ of $L^{2}(M)$ such that $r \mathcal{H} r \neq\{0\}$. Let $D(\delta) \subset M$ be $a *$-subalgebra which contains $M_{0}$ and $B$.

Assume that there exists a real derivation $\delta: D(\delta) \rightarrow L^{2}\left(\left\langle M, e_{B}\right\rangle\right)$ such that $\delta_{\mid M_{0}}$ is unbounded, $\delta^{*}\left(e_{B}\right)$ exists and belongs to $M_{0}$, and $\delta(b)=0$, for all $b \in B$.

Also, suppose that $M_{0}$ is finitely generated and $\delta\left(M_{0}\right) \subset$ span $M_{0} e_{B} M_{0}$. More generally, suppose that $M_{0}=\bigcup_{n \geqslant 1} M_{n}$, where $M_{n}$ is a finitely generated *subalgebra such that $M_{n} \subset M_{n+1}$ and $\delta\left(M_{n}\right) \subset$ span $M_{n} e_{B} M_{n}$, for all $n \geqslant 1$.

Then $M$ has no Cartan subalgebra and does not have property Gamma.

The mixingness condition was inspired by [Ho14, Corollary C], where it is shown that if an orthogonal representation $\pi: G \rightarrow \mathcal{O}\left(H_{\mathbb{R}}\right)$ contains a mixing subrepresentation, then the $\mathrm{II}_{1}$ factor $\Gamma\left(H_{\mathbb{R}}\right)^{\prime \prime} \rtimes G$ associated to the corresponding free Bogoljubov action has no Cartan subalgebra.

Before proceeding to the proof of Theorem 7.1, let us derive several consequences of it. Firstly, note that Corollary 1.4 corresponds precisely to the case $B=\mathbb{C} 1$. Secondly, let us deduce Corollary 1.6.

7.1. Proof of Corollary 1.6. Recall that $M=M_{1} *_{B} M_{2}$. Let $D(\delta)$ be the *algebra generated by $M_{1}$ and $M_{2}$ and define $\delta: D(\delta) \rightarrow L^{2}\left(\left\langle M, e_{B}\right\rangle\right)$ by letting $\delta(x)=i\left[x, e_{B}\right]$ if $x \in M_{1}$, and $\delta(x)=0$ if $x \in M_{2}$. Then it is easy to see that $\delta$ is a real derivation and $\delta^{*}\left(e_{B}\right)=0$.

Let $M_{1, n} \subset M_{1}$ and $M_{2, n} \subset M_{2}$ be increasing sequences of finitely generated *-subalgebras such that $M_{1,0}=\bigcup_{n \geqslant 1} M_{1, n}$ is weakly dense in $M_{1}$ and $M_{2,0}=$ $\bigcup_{n \geqslant 1} M_{2, n}$ is weakly dense in $M_{2}$. Assume that $u \in M_{1,1}$ and $v \in M_{2,1}$. Denote by $M_{n}$ the algebra generated by $M_{1, n}$ and $M_{2, n}$. Then $M_{0}=\bigcup_{n \geqslant 1} M_{n}$ is weakly dense in $M$ and $\delta\left(M_{n}\right) \subset \operatorname{span} M_{n} e_{B} M_{n}$, for all $n \geqslant 1$. Since $u, v$ are unitaries, $u \in M_{1} \ominus B$ and $v \in M_{2} \ominus B$, it follows that $\left\|\delta\left((u v)^{n}\right)\right\|_{2}=\sqrt{2 n}$, for all $n \geqslant 1$. This shows that $\delta_{\mid M_{0}}$ is unbounded. Thus, $\delta$ satisfies all the assumptions required in Theorem 7.1 .

We continue by verifying the rest of the assumptions from Theorem 7.1

We first claim that $M$ is a factor and does not have property Gamma. Let $x \in M^{\prime} \cap M^{\omega}$. Since $E_{B}(u)=E_{B}(v)=E_{B}(w)=E_{B}\left(w^{*} v\right)=0$, Io15, Lemma 6.1] gives that $M^{\prime} \cap M^{\omega} \subset B^{\omega}$, and thus $x \in B^{\omega}$. Recall that $z B z^{*} \perp B$, for some $z \in\{u, v\}$. Therefore, $\left\langle z(x-\tau(x)) z^{*}, x-\tau(x)\right\rangle=0$. On the other hand, since $x$ commutes with $z$, we have that $z(x-\tau(x)) z^{*}=x-\tau(x)$. Altogether, it follows that $x=\tau(x) \in \mathbb{C} 1$, thereby proving the claim.

Next, using the same argument as in the proof of [Io15, Lemma 6.1], we prove that $S=\{u, v, w\}$ is a non-amenability set for $M$ relative to $B$. For $i \in\{1,2\}$, we denote by $W_{i} \subset M$ the set of alternating words in $M_{1} \ominus B$ and $M_{2} \ominus B$ which start in $M_{i} \ominus B$, and by $\mathcal{H}_{i} \subset L^{2}\left(\left\langle M, e_{B}\right\rangle\right)$ the $\|.\|_{2}$-closure of the linear span of $W_{i} e_{B} M$. Let $\mathcal{H}_{0} \subset L^{2}\left(\left\langle M, e_{B}\right\rangle\right)$ be the $\|\cdot\|_{2}$-closure of $e_{B} M$. Then $L^{2}\left(\left\langle M, e_{B}\right\rangle\right)=\mathcal{H}_{0} \oplus \mathcal{H}_{1} \oplus \mathcal{H}_{2}$. For $i \in\{0,1,2\}$, let $e_{i}$ be the orthogonal projection onto $\mathcal{H}_{i}$.

Notice that if $x \in M_{1} \ominus B$ and $y \in M_{2} \ominus B$, then $x \mathcal{H}_{2} x^{*} \subset \mathcal{H}_{1}$ and $y \mathcal{H}_{1} y^{*} \subset \mathcal{H}_{2}$. Since $E_{B}(u)=E_{B}(v)=E_{B}(w)=E_{B}\left(w^{*} v\right)=0$, we deduce that

$$
u \mathcal{H}_{2} u^{*} \subset \mathcal{H}_{1}, \quad v \mathcal{H}_{1} v^{*} \subset \mathcal{H}_{2}, \quad w \mathcal{H}_{1} w^{*} \subset \mathcal{H}_{2} \text { and } v \mathcal{H}_{1} v^{*} \perp w \mathcal{H}_{1} w^{*} \text {. }
$$

Now, let $\xi \in L^{2}\left(\left\langle M, e_{B}\right\rangle\right)$ and denote $C_{\xi}=\sum_{z \in S}\left\|z \xi z^{*}-\xi\right\|_{2}$. Then equation (7.1) implies that

$$
\left\|e_{2}\left(u^{*} \xi u\right)\right\|_{2} \leqslant\left\|e_{1}(\xi)\right\|_{2} \text { and }\left\|e_{1}\left(v^{*} \xi v\right)\right\|_{2}^{2}+\left\|e_{1}\left(w^{*} \xi w\right)\right\|_{2}^{2} \leqslant\left\|e_{2}(\xi)\right\|_{2}^{2}
$$


These inequalities further imply that

$$
\left\|e_{2}(\xi)\right\|_{2}-C_{\xi} \leqslant\left\|e_{1}(\xi)\right\|_{2} \text { and } \sqrt{2}\left(\left\|e_{1}(\xi)\right\|_{2}-C_{\xi}\right) \leqslant\left\|e_{2}(\xi)\right\|_{2} \text {. }
$$

From this it is easy to derive that $\left\|e_{1}(\xi)\right\|_{2} \leqslant 6 C_{\xi}$ and $\left\|e_{2}(\xi)\right\|_{2} \leqslant 7 C_{\xi}$. Since $u \mathcal{H}_{0} u^{*} \subset \mathcal{H}_{1}$, we similarly get that $\left\|e_{0}(\xi)\right\|_{2} \leqslant\left\|e_{1}(\xi)\right\|_{2}+C_{\xi} \leqslant 7 C_{\xi}$. Altogether, it follows that $\|\xi\|_{2} \leqslant 20 C_{\xi}$, proving that $S$ is indeed a non-amenability set relative to $B$.

Finally, let $r \in M$ be a non-zero projection. We claim that there exists a mixing $B$ - $B$ bimodule $\mathcal{H} \subset L^{2}(M)$ such that $r \mathcal{H} r \neq\{0\}$. Assume by contradiction that this is false. Let $z_{1}, z_{2} \in\{u, v\}$ be such that $z_{1} B z_{1}^{*} \perp B$ and $\left\{z_{1}, z_{2}\right\}=\{u, v\}$. Denote $z=z_{2} z_{1}$. Then for every $k \geqslant 1$, by using freeness, it is clear that $z^{k} B z^{k^{*}} \perp B$. Thus, the $B-B$ bimodule $\mathcal{H}_{k}=\overline{B z^{k} B}\|\cdot\|_{2}$ is isomorphic to the coarse $B$ - $B$ bimodule, $L^{2}(B) \bar{\otimes} L^{2}(B)$, and is therefore mixing.

By our assumption we have that $r \mathcal{H}_{k} r=\{0\}$, hence $r z^{k} r=0$, for all $k \geqslant 1$. In particular, we get that $\left(\frac{1}{n} \sum_{k=1}^{n} z^{k^{*}} r z^{k}\right) r=0$, for all $n \geqslant 1$. Let $D \subset M$ denote the von Neumann subalgebra generated by $z$. Von Neumann's ergodic theorem implies that $\frac{1}{n} \sum_{k=1}^{n} z^{k^{*}} r z^{k}$ converges in $\|\cdot\|_{2}$ to $E_{D^{\prime} \cap M}(r)$, as $n \rightarrow \infty$. Thus, we derive that $E_{D^{\prime} \cap M}(r) r=0$ and further that $\left(E_{D^{\prime} \cap M}(r)\right)^{2}=0$. Since $r \geqslant 0$ it follows that $E_{D^{\prime} \cap M}(r)=0$ and $\tau(r)=\tau\left(E_{D^{\prime} \cap M}(r)\right)=0$, hence $r=0$. This provides the desired contradiction.

Altogether, we can apply Theorem 7.1 and derive the conclusion.

In the proof of Theorem 7.1 we will need the following technical result which says that if $L^{2}(M)$ contains "enough" mixing $B-B$ bimodules, then no Cartan subalgebra of $M$ can be embedded into $B$. More precisely, we have

Proposition 7.2. Let $M$ be a $I I_{1}$ factor and $B \subset M$ be a von Neumann subalgebra. Assume that for any non-zero projection $r \in B^{\prime} \cap M$, there exists a mixing $B$-B sub-bimodule $\mathcal{H}$ of $L^{2}(M)$ such that $r \mathcal{H} r \neq\{0\}$.

(1) If $A \subset M$ is a Cartan subalgebra, then $A \nprec_{M} B$.

(2) If $M$ has property $\Gamma$, then $M^{\prime} \cap M^{\omega} \nprec_{M^{\omega}} B^{\omega}$. Moreover, in this case, let $\left(N, \tau^{\prime}\right)$ be any tracial von Neumann algebra containing $B$ such that $\tau_{\mid B}=$ $\tau^{\prime}{ }_{B B}$ and denote $\tilde{M}=M *_{B} N$. Then $M^{\prime} \cap M^{\omega} \nprec_{\tilde{M}^{\omega}} B^{\omega}$.

Proof. (1) Assume by contradiction that $A \subset M$ is a Cartan subalgebra such that $A \prec_{M} B$. Then we can find projections $p \in A, q \in B$, a non-zero partial isometry $v \in M$ and a $*$-homomorphism $\phi: A p \rightarrow q B q$ such that $v^{*} v=p, q_{0}=v v^{*} \leqslant q$ and $\phi(x) v=v x$, for all $x \in A p$.

Towards a contradiction, let $r \in B^{\prime} \cap M$ be the smallest projection such that $q_{0} \leqslant r$. By the hypothesis we can find a mixing $B$ - $B$ bimodule $\mathcal{H} \subset L^{2}(M)$ such that $r \mathcal{H} r \neq\{0\}$. Denote by $e$ the orthogonal projection from $L^{2}(M)$ onto $\mathcal{H}$.

Denote $B_{0}=\phi(A p) \subset q B q$. Fix $u \in \mathcal{N}_{p M p}(A p)$ and denote by $\theta$ the automorphism of $B_{0}$ given by $\theta=\phi \circ \operatorname{Ad}(u) \circ \phi^{-1}$. Then we have that

$$
v u v^{*} y=\theta(y) v_{u v}^{*} \text { for all } y \in B_{0} \text {. }
$$

Since $B_{0}$ is diffuse, we can find a sequence $y_{n} \in \mathcal{U}\left(B_{0}\right)$ such that $y_{n} \rightarrow 0$, weakly. Since $\mathcal{H}$ is a $B-B$ bimodule by using equation (7.2) we get that $e\left(v u v^{*}\right) y_{n}=$ $\theta\left(y_{n}\right) e\left(v u v^{*}\right)$, for all $n$. Hence we have that $\left\langle\theta\left(y_{n}^{*}\right) e\left(v u v^{*}\right) y_{n}, e\left(v u v^{*}\right)\right\rangle=\left\|e\left(v u v^{*}\right)\right\|_{2}^{2}$, for all $n$. Since $y_{n} \rightarrow 0$ weakly and $\mathcal{H}$ is a mixing $B$ - $B$ bimodule, we derive that $e\left(v u v^{*}\right)=0$. 
Since this holds for any unitary $u \in \mathcal{N}_{p M p}(A p)$ and $A p \subset p M p$ is a Cartan subalgebra, we conclude that $e\left(q_{0} M q_{0}\right)=e\left(v p M p v^{*}\right)=\{0\}$. Let us show that this implies that $e(r M r)=\{0\}$.

Indeed, denote by $\mathcal{K}$ the $\|\cdot\|_{2}$ closure of the convex hull of the set $\left\{w q_{0} w^{*} \mid\right.$ $w \in \mathcal{U}(B)\}$. Since $e\left(q_{0} M q_{0}\right)=\{0\}$ and $e$ is the orthogonal projection onto a $B$ - $B$ bimodule, it follows that we have $e(z M z)=\{0\}$, for all $z \in \mathcal{K}$. Since $E_{B^{\prime} \cap M}\left(q_{0}\right) \in$ $\mathcal{K}$ (more precisely, $E_{B^{\prime} \cap M}\left(q_{0}\right)$ is the unique element of minimal $\|\cdot\|_{2}$ in $\mathcal{K}$ ) we get that $e\left(E_{B^{\prime} \cap M}\left(q_{0}\right) M E_{B^{\prime} \cap M}\left(q_{0}\right)\right)=\{0\}$. Since $r$ is equal to the support of $E_{B^{\prime} \cap M}\left(q_{0}\right)$, we have that $E_{B^{\prime} \cap M}\left(q_{0}\right) M E_{B^{\prime} \cap M}\left(q_{0}\right)$ is a $\|\cdot\|_{2^{-}}$-dense subspace of $r M r$. Thus, we would get that $e(r M r)=\{0\}$ or, equivalently, that $r \mathcal{H} r=\{0\}$, which provides the desired contradiction.

(2) First, assume by contradiction that $M$ has property Gamma and that $M^{\prime} \cap$ $M^{\omega} \prec_{M^{\omega}} B^{\omega}$.

Let $\left\{y_{i}\right\}_{i \geqslant 1}$ be a $\|\cdot\|_{2}$ dense sequence in $M$. Since $M$ has property Gamma, by a construction of Popa (see the proof of [Oz04, Proposition 7]) we can find diffuse abelian von Neumann subalgebras $\left\{A_{n}\right\}_{n \geqslant 1}$ of $M$ such that for all $n$ we have that $A_{n+1} \subset A_{n}$ and that

$$
\left\|y_{i}-E_{A_{n}^{\prime} \cap M}\left(y_{i}\right)\right\|_{2} \leqslant \frac{1}{n}, \text { for all } 1 \leqslant i \leqslant n .
$$

Then we have

Claim. $A_{n} \prec_{M} B$, for some $n \geqslant 1$.

Proof of the Claim. Denote by $A_{\omega}=\prod_{n=1}^{\omega} A_{n}$ the von Neumann subalgebra of $M^{\omega}$ consisting of all $x=\left(x_{n}\right)_{n}$ such that $\lim _{n \rightarrow \omega}\left\|x_{n}-E_{A_{n}}\left(x_{n}\right)\right\|_{2}=0$.

Then (7.3) implies that $A_{\omega} \subset M^{\prime} \cap M^{\omega}$. Since $M^{\prime} \cap M^{\omega} \prec_{M^{\omega}} B^{\omega}$, we get that $A_{\omega} \prec_{M^{\omega}} B^{\omega}$. Thus, we can find projections $p \in A_{\omega}, q \in B^{\omega}$, a non-zero partial isometry $v \in q M^{\omega} r$ and a $*$-homomorphism $\phi: A_{\omega} p \rightarrow q B^{\omega} q$ such that $\phi(x) v=v x$, for all $x \in A_{\omega} r$.

Let $\delta=\left\|E_{B^{\omega}}\left(v v^{*}\right)\right\|_{2}$. Then $\left\|E_{B^{\omega}}\left(v u v^{*}\right)\right\|_{2}=\delta$, for all $u \in \mathcal{U}\left(A_{\omega} p\right)$. Write $p=\left(p_{n}\right)_{n}$ and $v=\left(v_{n}\right)_{n}$, where $p_{n} \in A_{n}$ is a projection and $v_{n} \in M$, for all $n$.

If the claim is false, then $A_{n} \nprec_{M} B$ and thus $A_{n} p_{n} \nprec_{M} B$, for all $n \geqslant 1$. Thus, for every $n \geqslant 1$, we can find a unitary $u_{n} \in A_{n} p_{n}$ such that $\left\|E_{B}\left(v_{n} u_{n} v_{n}^{*}\right)\right\|_{2} \leqslant \frac{\delta}{2}$. Then the unitary $u=\left(u_{n}\right) \in A_{\omega} p$ satisfies $\left\|E_{B^{\omega}}\left(v u v^{*}\right)\right\|_{2}=\lim _{n \rightarrow \omega}\left\|E_{B}\left(v_{n} u_{n} v_{n}^{*}\right)\right\|_{2} \leqslant \frac{\delta}{2}$, which gives a contradiction.

Let $n$ be such that $A_{n} \prec_{M} B$. Then we can find projections $p \in A_{n}, q \in B$, a non-zero partial isometry $v \in q M p$ and a $*$-homomorphism $\phi: A_{n} p \rightarrow q B q$ such that $\phi(x) v=v x$, for all $x \in A_{n} p$. Denote $q_{0}=v v^{*} \leqslant q$ and let $r \in B^{\prime} \cap M$ be the smallest projection such that $q_{0} \leqslant r$. The hypothesis implies the existence of a non-zero mixing $B$ - $B$ bimodule $\mathcal{H} \subset L^{2}(M)$ such that $r \mathcal{H} r \neq\{0\}$. Denote by $e$ the orthogonal projection from $L^{2}(M)$ onto $\mathcal{H}$.

Now, let $N \geqslant n, u \in A_{N}^{\prime} \cap M$ and $x \in A_{N} p$. Write $x=x_{0} p$, where $x_{0} \in A_{N}$. Since $u$ and $x_{0}$ commute and $v=v p$ we get that $v u v^{*} \phi(x)=v u x v^{*}=v u x_{0} v^{*}=$ $v x_{0} u v^{*}=v x u v^{*}=\phi(x) v u v^{*}$. This shows that $v\left(A_{N}^{\prime} \cap M\right) v^{*} \subset q_{0} M q_{0}$ commutes with $\phi\left(A_{N} p\right) \subset q B q$.

Since $\mathcal{H}$ is a mixing $B-B$ bimodule and $A_{N}$ is diffuse, by repeating the argument from the proof of (1) we get that $e\left(v\left(A_{N}^{\prime} \cap M\right) v^{*}\right)=\{0\}$, for all $N \geqslant n$. Equation (7.3) then implies that $e\left(v y_{i} v^{*}\right)=0$, for all $i \geqslant 1$. By using the $\|\cdot\|_{2}$ density of 
$\left\{y_{i}\right\}_{i \geqslant 1}$ in $M$ we conclude that $e\left(q_{0} M q_{0}\right)=e\left(v M v^{*}\right)=\{0\}$ and the end of the proof of (1) yields a contradiction.

To prove the moreover assertion, assume by contradiction that $M^{\prime} \cap M^{\omega} \prec_{\tilde{M}^{\omega}}$ $B^{\omega}$. Then the above claim implies that $A_{n} \prec_{\tilde{M}} B$, for some $n \geqslant 1$. Since $A_{n} \subset M$ and $\tilde{M}=M *_{B} N$, by [PP08, Theorem 1.2.1] we get that $A_{n} \prec_{M} B$. Continuing as above yields a contradiction.

7.2. Proof of Theorem [7.1. Define $\tilde{M}=M *_{B}(B \bar{\otimes} L(\mathbb{Z}))$ and let $\left\{\alpha_{t}\right\}_{t \in \mathbb{R}}$ be the one-parameter group of automorphisms of $\tilde{M}$ arising from $\delta$ as provided by Proposition 3.3. Note that since $\delta_{\mid B} \equiv 0$, we have that $\alpha_{t}(x)=x$, for all $x \in B$ and every $t \in \mathbb{R}$. Let $S \subset M_{0}$ be a non-amenability set for $M$ relative to $B$.

The core of the proof consists of proving several claims about the inclusion $M \subset \tilde{M}$.

Claim 1. $\delta_{\mid M_{0}}$ is unbounded.

Proof of Claim 1. Let $s \in L(\mathbb{Z})$ be a semicircular element and $L^{2}\left(\left\langle M, e_{B}\right\rangle\right) \ni \xi \rightarrow$ $\xi \# s \in L^{2}(\tilde{M})$ be the unique embedding of $M-M$ bimodules sending $e_{B}$ to $s$. Let $a, b, c \in M_{0}$. Since $\delta$ is a real derivation, a calculation in the spirit of the proof of [Vo98b, Proposition 4.1] gives that

$$
\begin{gathered}
\left\langle a \delta^{*}\left(e_{B}\right) b-E_{M}((\delta(a) \# s) s) b-a E_{M}(s(\delta(b) \# s)), c\right\rangle \\
=\left\langle\delta^{*}\left(e_{B}\right), a^{*} c b^{*}\right\rangle-\left\langle\delta(a) \# s, c b^{*} s\right\rangle-\left\langle\delta(b) \# s, s a^{*} c\right\rangle \\
=\left\langle\delta\left(b c^{*} a\right)-b c^{*} \delta(a)-\delta(b) c^{*} a, e_{B}\right\rangle=\left\langle b \delta\left(c^{*}\right) a, e_{B}\right\rangle=\left\langle a e_{B} b, \delta(c)\right\rangle .
\end{gathered}
$$

Thus, $a e_{B} b$ belongs to the domain of $\delta^{*}$, for all $a, b \in M_{0}$. Hence, for all $x \in D(\delta)$, we have that

$$
\|\delta(x)\|_{2}=\sup _{y \in \operatorname{span}\left(M_{0} e_{B} M_{0}\right),\|y\|_{2} \leqslant 1}|\langle\delta(x), y\rangle|=\sup _{y \in \operatorname{span}\left(M_{0} e_{B} M_{0}\right),\|y\|_{2} \leqslant 1}\left|\left\langle x, \delta^{*}(y)\right\rangle\right| .
$$

Since $\delta$ is unbounded and $M_{0} \subset D(\delta)$ is dense in $\|\cdot\|_{2}$, it follows that $\delta_{\mid M_{0}}$ is unbounded.

Claim 2. $M^{\prime} \cap \tilde{M}^{\omega} \subset M^{\omega}$.

Proof of Claim 2. Since $\tilde{M}=M *_{B}(B \bar{\otimes} L(\mathbb{Z}))$, there exists a $B-M$ bimodule $\mathcal{K}$ such that as $M-M$ bimodules we have $L^{2}(\tilde{M}) \ominus L^{2}(M) \cong L^{2}(M) \otimes_{B} \mathcal{K}$. Since $S$ is a non-amenability set for $M$ relative to $B$, the second part of Lemma 2.6 implies that there is $\kappa>0$ such that $\|\xi\|_{2} \leqslant \kappa \sum_{y \in S}\|y \xi-\xi y\|_{2}$, for all $\xi \in L^{2}(\tilde{M}) \ominus L^{2}(M)$. This gives that $M^{\prime} \cap \tilde{M}^{\omega} \subset M^{\omega}$.

Note that since $M$ is a factor, Claim 2 implies that $M^{\prime} \cap \tilde{M}=\mathbb{C} 1$.

Claim 3. $\alpha_{t}(M)$ is not amenable relative to $B$ inside $\tilde{M}$, and $\alpha_{t}(M) \nprec_{\tilde{M}} B \bar{\otimes} L(\mathbb{Z})$, for any $t \in \mathbb{R}$.

Proof of Claim 3. Consider the $B$ - $M$ bimodule $\mathcal{H}=\mathcal{K} \otimes_{B} L^{2}(\tilde{M})$, where $\mathcal{K}$ is as in the proof of Claim 2. Then we have that $L^{2}\left(\left\langle\tilde{M}, e_{B}\right\rangle\right) \cong L^{2}(\tilde{M}) \otimes_{B} L^{2}(\tilde{M}) \cong$ $L^{2}(M) \otimes_{B}\left(L^{2}(\tilde{M}) \oplus \mathcal{H}\right)$, as $M-M$ bimodules. The second part of Lemma 2.6 now implies that $S$ is a non-amenability set for $M$ relative to $B$ inside $\tilde{M}$. In particular, $M$ is not amenable relative to $B$ inside $\tilde{M}$. Since $\alpha_{t}$ leaves $B$ invariant, we derive that $\alpha_{t}(M)$ is not amenable relative to $B$ inside $\tilde{M}$, for any $t \in \mathbb{R}$. 
Assume by contradiction that $\alpha_{t}(M) \prec_{\tilde{M}} B \bar{\otimes} L(\mathbb{Z})$, for some $t \in \mathbb{R}$. Since $\alpha_{t}(M)^{\prime} \cap \tilde{M}=\mathbb{C} 1$, by [Io15, Remark 2.2] it follows that $\alpha_{t}(M)$ is amenable relative to $B \bar{\otimes} L(\mathbb{Z})$ inside $\tilde{M}$. Note that $B \bar{\otimes} L(\mathbb{Z})$ is amenable relative to $B$ inside $\tilde{M}$. Indeed, if $u \in L(\mathbb{Z})$ is a generating Haar unitary, then the vectors $\xi_{n}=\frac{1}{\sqrt{n}} \sum_{k=1}^{n} u^{k} e_{B} u^{k^{*}} \in L^{2}\left(\left\langle\tilde{M}, e_{B}\right\rangle\right)$ satisfy $\left\langle x \xi_{n}, \xi_{n}\right\rangle=\tau(x)$, for all $x \in \tilde{M}$, and $\left\|y \xi_{n}-\xi_{n} y\right\|_{2} \rightarrow 0$, for all $y \in B \bar{\otimes} L(\mathbb{Z})$.

By combining the last two facts and using OP10, Proposition 2.4 (3)] we deduce that $\alpha_{t}(M)$ is amenable relative to $B$ inside $\tilde{M}$. This leads to a contradiction.

Claim 4. There exists $t_{0}>0$ such that $\alpha_{t}(M) \nprec_{\tilde{M}} M$, for all $t \in\left(0, t_{0}\right)$.

Proof of Claim 4. Assuming that the claim is false, we can find a sequence $t_{n} \rightarrow 0$ with $t_{n}>0$ such that $\alpha_{t_{n}}(M) \prec_{\tilde{M}} M$, for all $n \geqslant 1$. On the other hand, Claim 3 gives that $\alpha_{t_{n}}(M) \nprec_{\tilde{M}} B$. Recall that $\alpha_{t_{n}}(M)^{\prime} \cap \tilde{M}=\mathbb{C} 1, M$ is a factor and $\tilde{M}=M *_{B}(B \bar{\otimes} L(\mathbb{Z}))$. By combining all these facts, the proof of IPP08, Theorem $5.1]$ implies that we can find a unitary operator $v_{n} \in \tilde{M}$ such that $v_{n} \alpha_{t_{n}}(M) v_{n}^{*} \subset M$.

Since $S$ is a non-amenability set for $M$ relative to $B$ inside $\tilde{M}$, Lemma 2.7 (2) provides a constant $C>0$ such that for every $n \geqslant 1$ we have

$$
\left\|\alpha_{t_{n}}(x)-E_{M}\left(\alpha_{t_{n}}(x)\right)\right\|_{2} \leqslant C \sum_{y \in S}\left\|\alpha_{t_{n}}(y)-y\right\|_{2}, \text { for all } x \in(M)_{1} .
$$

Finally, let $x \in M_{0}$. Proposition 3.3 gives that $\left\|\frac{\alpha_{t}(x)-x}{t}-\delta(x) \# s\right\|_{2} \rightarrow 0$, as $t \rightarrow$ 0 . Also, we have that $\|\delta(x) \# s\|_{2}=\|\delta(x)\|_{2}$ and $E_{M}(\delta(x) \# s)=0$. By combining these facts with the last inequality we get that $\|\delta(x)\|_{2} \leqslant C \sum_{y \in S}\|\delta(y)\|_{2}$, for all $x \in M_{0}$, which contradicts Claim 1 .

Claim 5. $M$ does not have property Gamma.

Proof of Claim 5. Assume by contradiction that $M$ has property Gamma and let $t \in\left(0, t_{0}\right)$, where $t_{0}$ is given by Claim 4. Proposition 2.11 (2) then implies that $M^{\prime} \cap M^{\omega} \nprec_{\tilde{M}^{\omega}} B^{\omega}$. Since $B$ is invariant under $\alpha_{t}$, we get that $\alpha_{t}(M)^{\prime} \cap \tilde{M}^{\omega} \nprec_{\tilde{M}^{\omega}}$ $B^{\omega}$. Also, by the above claims we have that $\alpha_{t}(M) \nprec_{\tilde{M}} B \bar{\otimes} L(\mathbb{Z})$ and $\alpha_{t}(M) \nprec_{\tilde{M}} M$.

By applying [Io15, Theorem 6.3] to the inclusion $\alpha_{t}(M) \subset \tilde{M}=M *_{B}(B \bar{\otimes} L(\mathbb{Z}))$, we deduce that $\alpha_{t}(M) p$ is amenable relative to $B$ inside $\tilde{M}$, for a non-zero projection $p \in \alpha_{t}(M)^{\prime} \cap \tilde{M}$. Since $\alpha_{t}(M)^{\prime} \cap \tilde{M}=\mathbb{C} 1$, this would imply that $\alpha_{t}(M)$ is amenable relative to $B$ inside $\tilde{M}$, which is false, by Claim 3 .

We are now ready to prove the conclusion of Theorem 7.1 Thus, assume by contradiction that $M$ has a Cartan subalgebra $A$. Let $t \in\left(0, t_{0}\right)$. Note that $\alpha_{t}(M) \subset \mathcal{N}_{\tilde{M}}\left(\alpha_{t}(A)\right)^{\prime \prime}$ and therefore $\alpha_{t}(M) \subset \mathcal{N}_{\tilde{M}}\left(\alpha_{t}(A)\right)^{\prime \prime}$. By combining Claims 2 and 5, we get that $M^{\prime} \cap \tilde{M}^{\omega}=\mathbb{C} 1$. Thus, we also have that $\alpha_{t}(M)^{\prime} \cap \tilde{M}^{\omega}=\mathbb{C} 1$. Altogether, we are in position to apply Theorem 2.12 (in the case $Q=\mathbb{C} 1$ ) and deduce that one of the following conditions holds:

(1) $\alpha_{t}(A) \prec_{\tilde{M}} B$.

(2) $\alpha_{t}(M) \prec_{\tilde{M}} M$.

(3) $\alpha_{t}(M) \prec_{\tilde{M}} B \bar{\otimes} L(\mathbb{Z})$.

(4) $\alpha_{t}(M)$ is amenable relative to $B$ inside $\tilde{M}$. 
Since $\alpha_{t}$ leaves $B$ invariant, condition (1) implies that $A \prec_{\tilde{M}} B$. Since $A \subset M$, [PP08, Theorem 1.2.1] implies that $A \prec_{M} B$. This however cannot happen by Proposition 2.11 (1). Since conditions (2)-(4) are also false as shown above, we get a contradiction.

\section{Algebraic Cocycles and uniqueness of CARtan subalgebras}

In this final section, we first prove a slightly more general form of Theorem 1.7 and then derive Corollary 1.8 .

Theorem 8.1. Let $\Gamma$ be a group satisfying all the assumptions from Theorem 1.7 . Assume that $\Gamma^{\prime}$ is a group which admits a finite normal subgroup $N$ such that $\Gamma^{\prime} / N \cong \Gamma$.

Then $L^{\infty}(X)$ is the unique Cartan subalgebra of $L^{\infty}(X) \rtimes \Gamma^{\prime}$, up to unitary conjugacy, for any free ergodic probability measure preserving action $\Gamma^{\prime} \curvearrowright(X, \mu)$.

Proof. Let $\Lambda<\Gamma$ be a subgroup and $b: \Gamma \rightarrow \mathbb{C}(\Gamma / \Lambda)$ a cocycle satisfying the hypothesis of Theorem 1.7. After replacing $b$ with its real or imaginary part, we may assume that we have $b(\Gamma) \subset \mathbb{R}(\Gamma / \Lambda)$. For $g \in \Gamma$, write $b(g)=\sum_{h \Lambda \in \Gamma / \Lambda} c_{g, h \Lambda} \delta_{h \Lambda}$, where $c_{g, h \Lambda} \in \mathbb{R}$.

Consider the isometry $V: \ell^{2}(\Gamma / \Lambda) \rightarrow L^{2}\left(\left\langle L(\Gamma), e_{L(\Lambda)}\right\rangle\right)$ given by $V\left(\delta_{h \Lambda}\right)=$ $u_{h} e_{L(\Lambda)} u_{h}^{*}$. Then $V(\pi(g) \xi)=u_{g} \xi u_{g}^{*}$, where $\pi: \Gamma \rightarrow \ell^{2}(\Gamma / \Lambda)$ is the quasi-regular representation $\pi(g)\left(\delta_{h \Lambda}\right)=\delta_{g h \Lambda}$. itly,

We define $\delta: \mathbb{C} \Gamma \rightarrow L^{2}\left(\left\langle L(\Gamma), e_{L(\Lambda)}\right\rangle\right)$ by putting $\delta\left(u_{g}\right)=i V(b(g)) u_{g}^{*}$ or, explic-

$$
\delta\left(u_{g}\right)=i \sum_{h \Lambda \in \Gamma / \Lambda} c_{g, h \Lambda} u_{h} e_{L(\Lambda)} u_{h}^{*} u_{g}, \text { for all } g \in \Gamma \text {. }
$$

Then it is easy to see that $\delta$ is a real derivation. Since $b_{\mid \Lambda} \equiv 0$, we have that $\delta_{\mid \mathbb{C} \Lambda} \equiv 0$. As for every $g \in \Gamma$ we have that $\operatorname{Tr}\left(\delta\left(u_{g}\right) e_{L(\Lambda)}\right)=\delta_{g, e} c_{e, e \Lambda}=\delta_{g, e}\left\langle b(e), \delta_{e \Lambda}\right\rangle=0$, it follows that $\delta^{*}\left(e_{L(\Lambda)}\right)=0$. Since $b\left(\Gamma_{n}\right) \subset \mathbb{C}\left(\Gamma_{n} \Lambda / \Lambda\right)$, we get that $\delta\left(\mathbb{C} \Gamma_{n}\right) \subset$ $\operatorname{span}\left(\mathbb{C} \Gamma_{n} e_{L(\Lambda)} \mathbb{C} \Gamma_{n}\right)$, for all $n \geqslant 1$. Since $\mathbb{C} \Gamma_{n}$ is finitely generated and $\mathbb{C} \Gamma=$ $\bigcup_{n \geqslant 1} \mathbb{C} \Gamma_{n}$, we are in position to apply Proposition 3.3.

Let $\tilde{\Gamma}=\Gamma *_{\Lambda}(\Lambda \times \mathbb{Z})$ and let $s \in L(\mathbb{Z})$ be a generating semicircular element. Proposition 3.3 provides a one-parameter group of automorphisms $\left\{\alpha_{t}\right\}_{t \in \mathbb{R}}$ of $L(\tilde{\Gamma})$ satisfying $\left\|\frac{\alpha_{t}\left(u_{g}\right)-u_{g}}{t}-\delta\left(u_{g}\right) \# s\right\|_{2} \rightarrow 0$, for all $g \in \Gamma$. Since $\delta_{\mid \mathbb{C} \Lambda} \equiv 0$ and $\delta^{*}\left(e_{L(\Lambda)}\right)=$ 0 , we have that $\alpha_{t}(x)=x$, for all $x \in L(\Lambda \times \mathbb{Z})$.

For $f \in \ell^{\infty}(\Gamma / \Lambda)$ and $h \in \Gamma$, we define $\sigma(h)(f) \in \ell^{\infty}(\Gamma / \Lambda)$ by letting $\sigma(h)(f)(g \Lambda)$ $=f\left(h^{-1} g \Lambda\right)$. Since $\Lambda$ is not co-amenable in $\Gamma$, there exists a finite set $S \subset \Gamma$ such that there is no $\sigma(S)$-invariant state on $\ell^{\infty}(\Gamma / \Lambda)$. Consider the unital $*$ homomorphism $\rho: \ell^{\infty}(\Gamma / \Lambda) \rightarrow\left\langle L(\Gamma), e_{L(\Lambda)}\right\rangle$ given by

$$
\rho(f)=\sum_{g \Lambda \in \Gamma / \Lambda} f(g \Lambda) u_{g} e_{L(\Lambda)} u_{g}^{*}
$$

Then $\rho(\sigma(h)(f))=u_{h} \rho(f) u_{h}^{*}$, for all $h \in \Gamma$. Thus, there is no $S$-central state on $\left\langle L(\Gamma), e_{L(\Lambda)}\right\rangle$, and hence $S$ is a non-amenability set for $L(\Gamma)$ relative to $L(\Lambda)$.

Since $\delta$ is unbounded and $S \subset \Gamma$ is a non-amenability set for $L(\Gamma)$ relative to $L(\Lambda)$, Claims 1-3 from the proof of Theorem 7.1 (applied here verbatim in the case 
$M=L(\Gamma)$ and $B=L(\Lambda))$ give the following:

- $L(\Gamma)^{\prime} \cap L(\tilde{\Gamma})^{\omega} \subset L(\Gamma)^{\omega}$.

- $\alpha_{t}(L(\Gamma))$ is not amenable relative to $L(\Lambda)$ inside $L(\tilde{\Gamma})$, for any $t \in \mathbb{R}$.

- $\alpha_{t}(\Gamma) \nprec_{L(\tilde{\Gamma})} L(\Lambda \times \mathbb{Z})$, for any $t \in \mathbb{R}$.

- There exists $t_{0}>0$ such that $\alpha_{t}(L(\Gamma)) \nprec_{L(\tilde{\Gamma})} L(\Gamma)$, for any $t \in\left(0, t_{0}\right)$.

Now, let $\Gamma^{\prime} \curvearrowright(X, \mu)$ be a free ergodic pmp action. Define $M=L^{\infty}(X) \rtimes \Gamma^{\prime}$ and let $A$ be a Cartan subalgebra of $M$. We want to show that $A$ is unitarily conjugate to $L^{\infty}(X)$.

To this end, let $\Delta: M \rightarrow M \bar{\otimes} L(\Gamma)$ be the $*$-homomorphism given by $\Delta\left(a u_{g}\right)=$ $a u_{g} \otimes u_{p(g)}$, for all $a \in L^{\infty}(X)$ and $g \in \Gamma^{\prime}$ [PV10. Here, $p: \Gamma^{\prime} \rightarrow \Gamma$ denotes the quotient homomorphism. Further, we fix $t \in\left(0, t_{0}\right)$ and define $\theta_{t}=\left(\operatorname{id}_{M} \otimes \alpha_{t}\right) \circ \Delta$ : $M \rightarrow M \bar{\otimes} L(\tilde{\Gamma})$.

Then $\theta_{t}(M) \subset \mathcal{N}_{M \bar{\otimes} L(\tilde{\Gamma})}\left(\theta_{t}(A)\right)^{\prime \prime} ;$ therefore $u_{g} \otimes \alpha_{t}\left(u_{p(g)}\right) \in \mathcal{N}_{M \bar{\otimes} L(\tilde{\Gamma})}\left(\theta_{t}(A)\right)^{\prime \prime}$, for all $g \in \Gamma$. Since $L(\Gamma)^{\prime} \cap L(\tilde{\Gamma})^{\omega} \subset L(\Gamma)^{\omega}$ and $L(\Gamma)$ does not have property Gamma, we have $\alpha_{t}(L(\Gamma))^{\prime} \cap L(\tilde{\Gamma})^{\omega}=\mathbb{C} 1$. By applying Theorem 2.12 we conclude that one of the following conditions holds:

(1) $\theta_{t}(A) \prec_{M \bar{\otimes} L(\tilde{\Gamma})} M \bar{\otimes} L(\Lambda)$.

(2) $\alpha_{t}(L(\Gamma)) \prec_{L(\tilde{\Gamma})} L(\Gamma)$.

(3) $\alpha_{t}(L(\Gamma)) \prec_{L(\tilde{\Gamma})} L(\Lambda \times \mathbb{Z})$.

(4) $\alpha_{t}(L(\Gamma))$ is amenable relative to $L(\Lambda)$ inside $L(\tilde{\Gamma})$.

Since conditions (2)-(4) cannot hold by the above, condition (1) must be true. Since $\alpha_{t}$ leaves $L(\Lambda)$ invariant, (1) is equivalent to having $\Delta(A) \prec_{M \bar{\otimes} L(\tilde{\Gamma})} M \bar{\otimes} L(\Lambda)$. Since $\Delta(A) \subset M \bar{\otimes} L(\Gamma)$ and $L(\tilde{\Gamma})=L(\Gamma) *_{L(\Lambda)} L(\Lambda \times \mathbb{Z})$, [PP08, Theorem 1.2.1] gives that $\Delta(A) \prec_{M \bar{\otimes} L(\Gamma)} M \bar{\otimes} L(\Lambda)$.

By using [I015, Lemma 7.2] we derive that $A \prec_{M} L^{\infty}(X) \rtimes p^{-1}(\Lambda)$. For $i \in$ $\{1,2, \ldots, m\}$, let $g_{i}^{\prime} \in \Gamma^{\prime}$ such that $p\left(g_{i}^{\prime}\right)=g_{i}$. Since $M$ is a factor, HPV13, Proposition 8] implies that $A \prec_{M} L^{\infty}(X) \rtimes\left(\bigcap_{i=1}^{m} g_{i}^{\prime} p^{-1}(\Lambda) g_{i}^{\prime-1}\right)$. Since $\bigcap_{i=1}^{m} g_{i} \Lambda g_{i}^{-1}$ is finite, $\bigcap_{i=1}^{m} g_{i}^{\prime} p^{-1}(\Lambda) g_{i}^{\prime-1}$ is also finite. By combining the last two facts we get that $A \prec_{M} L^{\infty}(X)$. Since $A$ and $L^{\infty}(X)$ are Cartan subalgebras of $M$, Po06a, Theorem A.1] yields that they are unitarily conjugate (see also [Va07, Theorem C.3]).

Turning to the proof of Corollary 1.8, let us first establish the following technical result.

Lemma 8.2. Let $G$ be a countable group, $\Lambda<G$ be a subgroup, and $\theta: \Lambda \rightarrow G$ be an injective group homomorphism. Assume that $\Lambda \neq G$ and $\theta(\Lambda) \neq G$. Denote by $\Gamma=H N N(G, \Lambda, \theta)$ the corresponding $H N N$ extension. Then we have

(1) If $\bigcap_{i=1}^{m} h_{i} \Lambda h_{i}^{*}=\{e\}$, for some $h_{1}, h_{2}, \ldots, h_{m} \in \Gamma$, then $\Gamma$ is not inner amenable.

(2) $\Lambda$ is not co-amenable in $\Gamma$.

Remark 8.3. If $\Lambda=G$ or $\theta(\Lambda)=G$, then $\Lambda$ is co-amenable in $\Gamma$ by [MP3, Proposition 2].

Proof. Recall that $\Gamma=\left\langle G, t \mid t \lambda t^{-1}=\theta(\lambda), \forall \lambda \in \Lambda\right\rangle$, where $t$ is the so-called stable letter. Let $A \subset G$ and $B \subset G$ be sets of representatives of the left cosets of $\Lambda$ and $\theta(\Lambda)$ in $G$, respectively. We assume that $e \in A \cap B$. Since $\Lambda \neq G \neq \theta(\Lambda)$, we can find $a \in A \backslash\{e\}$ and $b \in B \backslash\{e\}$. 
Below, we will implicitly use the normal form theorem [LS77, Chapter IV, Theorem 2.1]: every $g \in \Gamma$ can be uniquely written as a product $g=g_{n} t^{\varepsilon_{n}} g_{n-1} \ldots g_{1} t^{\varepsilon_{1}} g_{0}$, for some $g_{0}, g_{1}, \ldots, g_{n} \in G$ and $\varepsilon_{1}, \ldots, \varepsilon_{n} \in\{-1,1\}$ such that $g_{i} \in A$ if $\varepsilon_{i}=-1$, and $g_{i} \in B$ if $\varepsilon_{i}=1$, for all $i \in\{1,2, \ldots, n\}$, and that there is no consecutive subsequence $t^{\varepsilon}, 1, t^{-\varepsilon}$ within the sequence $g_{n}, t^{\varepsilon_{n}}, \ldots, t^{\varepsilon_{1}}, g_{0}$.

(1) Assume by contradiction that $\Gamma$ is inner amenable and let $\phi: \ell^{\infty}(\Gamma \backslash\{e\}) \rightarrow \mathbb{C}$ be a state which is invariant under the conjugation action of $\Gamma$. For a subset $S \subset \Gamma$, we denote $m(S)=\phi\left(1_{S \backslash\{e\}}\right)$.

Denote by $S$ the set of all $g=g_{n} t^{\varepsilon_{n}} g_{n-1} \ldots g_{1} t^{\varepsilon_{1}} g_{0} \in \Gamma$ (represented in normal form) such that $n \geqslant 1$ and $g_{n} \neq e$. Also, denote by $U$ (respectively, $V$ ) the set of $g \in \Gamma$ such that $n \geqslant 1, g_{n}=e$ and $\varepsilon_{n}=-1$ (respectively, $\varepsilon_{n}=1$ ). Then we have that

$$
t^{-1} S t \subset U, \quad t S t^{-1} \subset V, \quad a U a^{-1} \subset S, \quad b V b^{-1} \subset S, \quad \text { and } a U a^{-1} \cap b V b^{-1}=\emptyset .
$$

Since $m$ is a $\Gamma$-invariant finitely additive measure, these inclusions imply that $m(S) \leqslant m(U), m(S) \leqslant m(V)$ and $m(U)+m(V) \leqslant m(S)$. From this we deduce that $m(S)=m(U)=m(V)=0$. Since $S \cup U \cup V \cup G=\Gamma$ and $m(\Gamma)=1$, we conclude that $m(G)=1$.

Since $t^{-1} G t \cap G=\Lambda$, we further get that $m(\Lambda)=1$. Finally, since $\bigcap_{i=1}^{m} h_{i} \Lambda h_{i}^{*}=$ $\{e\}$, we derive that $m(\{e\})=1$. This contradicts the fact that $m(\{e\})=0$.

(2) Assume by contradiction that $\Lambda$ is co-amenable inside $\Gamma$ and let $\phi: \ell^{\infty}(\Gamma / \Lambda) \rightarrow$ $\mathbb{C}$ be a $\Gamma$-invariant state. For a subset $S \subset \Gamma / \Lambda$, we denote $m(S)=\phi\left(1_{S}\right)$. Also, we denote by $\pi: \Gamma \rightarrow \Gamma / \Lambda$ the canonical projection, and still consider $U, V, S$ as in (1).

Next, we have that $t^{-1} S \subset U, t S \subset V, a U \subset S$ and $b V \subset S$. Moreover, $a \pi(U) \cap$ $b \pi(V)=\emptyset$. Since $\pi$ is $\Gamma$-equivariant and $m$ is a finitely additive $\Gamma$-invariant measure, it follows as above that $m(\pi(S))=m(\pi(U))=m(\pi(V))=0$. Since $\pi(S) \cup \pi(U) \cup$ $\pi(V) \cup G / \Lambda=\Gamma / \Lambda$ and $m(\Gamma / \Lambda)=1$, we conclude that $m(G / \Lambda)=1$. Finally, since $t G / \Lambda \cap G / \Lambda=\emptyset$, we would get that $m(\emptyset)=1$, which gives the desired contradiction.

8.1. Proof of Corollary 1.8. Let $\Gamma=\operatorname{HNN}(G, \Lambda, \theta)$. Since $\bigcap_{i=1}^{m} g_{i} \Lambda g_{i}^{-1}$ is finite, it follows that $N=\bigcap_{g \in \Gamma} g \Lambda g^{-1}$ is a finite normal subgroup of $\Gamma$ such that $N<\Lambda$. Moreover, we can find $h_{1}, h_{2}, \ldots, h_{n} \in \Gamma$ such that $N=\bigcap_{j=1}^{n} h_{j} \Lambda h_{j}^{-1}$. Denote $\Gamma_{0}=$ $\Gamma / N, G_{0}=G / N, \Lambda_{0}=\Lambda / N$ and let $p: \Gamma \rightarrow \Gamma_{0}$ be the quotient homomorphism.

Since the stable letter $t$ normalizes $N$, if $g \in \Lambda$, then $\theta(g)=\operatorname{tgt}^{-1} \in N$ if and only if $g \in N$. Therefore, $\theta: \Lambda \rightarrow G$ descends to an injective group homomorphism $\theta_{0}$ : $\Lambda_{0} \rightarrow G_{0}$. Moreover, we have that $\Gamma_{0}$ is naturally isomorphic to $\operatorname{HNN}\left(G_{0}, \Lambda_{0}, \theta_{0}\right)$ and $\bigcap_{j=1}^{n} p\left(h_{j}\right) \Lambda_{0} p\left(h_{j}\right)^{-1}=\{e\}$. Since $\Lambda_{0} \neq G_{0}$ and $\theta_{0}\left(\Lambda_{0}\right) \neq G_{0}$, by Lemma 8.2 we get that $\Gamma_{0}$ is not inner amenable (hence $L\left(\Gamma_{0}\right)$ is a $\mathrm{II}_{1}$ factor without property Gamma) and $\Lambda_{0}$ is not co-amenable inside $\Gamma_{0}$.

Next, we define $b: \Gamma_{0}=\operatorname{HNN}\left(G_{0}, \Lambda_{0}, \theta_{0}\right) \rightarrow \mathbb{C}\left(\Gamma_{0} / \Lambda_{0}\right)$ by letting $b(g)=0$, for all $g \in G_{0}$, and $b(t)=t \Lambda_{0}$, where $t \in \Gamma_{0}$ is the stable letter. Then $b$ is an unbounded cocycle. To see that $b$ is unbounded, just note that if $g \in G_{0} \backslash \Lambda_{0}$, then $\left\|b\left((g t)^{n}\right)\right\|_{2}=\sqrt{n}$, for all $n \geqslant 0$.

Finally, let $\left\{G_{n}\right\}_{n} \geqslant 1$ be a sequence of finitely generated subgroups of $G_{0}$ such that $G_{0}=\bigcup_{n \geqslant 1} G_{n}$. Let $\Gamma_{n}<\Gamma_{0}$ be the subgroup generated by $G_{n}$ and $t$. Then $\Gamma_{n}$ is finitely generated, $\Gamma_{n} \subset \Gamma_{n+1}$ and $b\left(\Gamma_{n}\right) \subset \mathbb{C}\left(\Gamma_{n} \Lambda / \Lambda\right)$, for all $n$. Moreover, $\bigcup_{n \geqslant 1} \Gamma_{n}=\Gamma_{0}$. Altogether, we can apply Theorem 8.1 to get the conclusion. 


\section{ACKNOWLEDGMENTS}

The authors are grateful to Cyril Houdayer, Jesse Peterson and Stefaan Vaes for helpful discussions and useful comments. They are also grateful to the referee for helpful suggestions.

\section{REFERENCES}

[AD95] C. Anantharaman-Delaroche, Amenable correspondences and approximation properties for von Neumann algebras, Pacific J. Math. 171 (1995), no. 2, 309-341. MR.1372231 (96m:46106)

[BCG03] P. Biane, M. Capitaine, and A. Guionnet, Large deviation bounds for matrix Brownian motion, Invent. Math. 152 (2003), no. 2, 433-459, DOI 10.1007/s00222-002-0281-4. MR 1975007 (2004g:60039)

[BV97] Mohammed E. B. Bekka and Alain Valette, Group cohomology, harmonic functions and the first $L^{2}$-Betti number, Potential Anal. 6 (1997), no. 4, 313-326, DOI 10.1023/A:1017974406074. MR1452785 (98e:20056)

[Be08] Serban Teodor Belinschi, The Lebesgue decomposition of the free additive convolution of two probability distributions, Probab. Theory Related Fields 142 (2008), no. 1-2, 125-150, DOI 10.1007/s00440-007-0100-3. MR2413268 (2009d:46115)

[BO08] Nathanial P. Brown and Narutaka Ozawa, $C^{*}$-algebras and finite-dimensional approximations, Graduate Studies in Mathematics, vol. 88, American Mathematical Society, Providence, RI, 2008. MR2391387 (2009h:46101)

[CS13] Ionut Chifan and Thomas Sinclair, On the structural theory of $\mathrm{II}_{1}$ factors of negatively curved groups (English, with English and French summaries), Ann. Sci. Éc. Norm. Supér. (4) 46 (2013), no. 1, 1-33 (2013). MR.3087388

[Co76] A. Connes, Classification of injective factors. Cases $I I_{1}, I I_{\infty}, I I I_{\lambda}, \lambda \neq 1$, Ann. of Math. (2) 104 (1976), no. 1, 73-115. MR.0454659 (56 \#12908)

[Da10a] Yoann Dabrowski, A note about proving non- $\Gamma$ under a finite non-microstates free Fisher information assumption, J. Funct. Anal. 258 (2010), no. 11, 3662-3674, DOI 10.1016/j.jfa.2010.02.010. MR2606868 (2011d:46135)

[Da10b] Yoann Dabrowski, A non-commutative path space approach to stationary free stochastic differential equations, preprint arXiv:1006.4351.

[Da14] Yoann Dabrowski, A free stochastic partial differential equation, Ann. Inst. Henri Poincaré Probab. Stat. 50 (2014), no. 4, 1404-1455, DOI 10.1214/13-AIHP548. MR.3270000

[DL92] E. Brian Davies and J. Martin Lindsay, Noncommutative symmetric Markov semigroups, Math. Z. 210 (1992), no. 3, 379-411, DOI 10.1007/BF02571804. MR.1171180|(93f:46088)

[FV12] Pierre Fima and Stefaan Vaes, HNN extensions and unique group measure space decomposition of $\mathrm{II}_{1}$ factors, Trans. Amer. Math. Soc. 364 (2012), no. 5, 2601-2617, DOI 10.1090/S0002-9947-2012-05415-8. MR2888221

[Ge98] Liming Ge, Applications of free entropy to finite von Neumann algebras. II, Ann. of Math. (2) 147 (1998), no. 1, 143-157, DOI 10.2307/120985. MR1609522 (99c:46068)

[Ho14] Cyril Houdayer, Structure of $\mathrm{II}_{1}$ factors arising from free Bogoljubov actions of arbitrary groups, Adv. Math. 260 (2014), 414-457, DOI 10.1016/j.aim.2014.04.010. MR 3209358

[HPV13] Cyril Houdayer, Sorin Popa, and Stefaan Vaes, A class of groups for which every action is $\mathrm{W}^{*}$-superrigid, Groups Geom. Dyn. 7 (2013), no. 3, 577-590. MR3095710

[Io12] Adrian Ioana, Classification and rigidity for von Neumann algebras, preprint arXiv:1212.0453, to appear in Proceedings of the 6th European Congress of Mathematics (Krakow, 2012), European Mathematical Society Publishing House.

[Io15] Adrian Ioana, Cartan subalgebras of amalgamated free product $\mathrm{II}_{1}$ factors (English, with English and French summaries), Ann. Sci. Éc. Norm. Supér. (4) 48 (2015), no. 1, 71-130. With an appendix by Ioana and Stefaan Vaes. MR.3335839

[IPP08] Adrian Ioana, Jesse Peterson, and Sorin Popa, Amalgamated free products of weakly rigid factors and calculation of their symmetry groups, Acta Math. 200 (2008), no. 1, 85-153, DOI 10.1007/s11511-008-0024-5. MR2386109(2009a:46119) 
[LS77] Roger C. Lyndon and Paul E. Schupp, Combinatorial group theory, Ergebnisse der Mathematik und ihrer Grenzgebiete, Band 89, Springer-Verlag, Berlin-New York, 1977. MR0577064 (58 \#28182)

[MP03] Nicolas Monod and Sorin Popa, On co-amenability for groups and von Neumann algebras (English, with French summary), C. R. Math. Acad. Sci. Soc. R. Can. 25 (2003), no. 3, 82-87. MR.1999183 (2004i:43004)

[MvN43] F. J. Murray and J. von Neumann, On rings of operators. IV, Ann. of Math. (2) 44 (1943), 716-808. MR0009096 (5,101a)

[OP10] Narutaka Ozawa and Sorin Popa, On a class of $\mathrm{II}_{1}$ factors with at most one Cartan subalgebra, Ann. of Math. (2) 172 (2010), no. 1, 713-749, DOI 10.4007/annals.2010.172.713. MR2680430 (2011j:46101)

[Oz04] Narutaka Ozawa, Solid von Neumann algebras, Acta Math. 192 (2004), no. 1, 111-117.

[Pe09a] Jesse Peterson, A 1-cohomology characterization of property $(T)$ in von Neumann algebras, Pacific J. Math. 243 (2009), no. 1, 181-199, DOI 10.2140/pjm.2009.243.181. MR2550142 (2010f:22009)

[Pe09b] Jesse Peterson, $L^{2}$-rigidity in von Neumann algebras, Invent. Math. 175 (2009), no. 2, 417-433, DOI 10.1007/s00222-008-0154-6. MR2470111 (2010b:46128)

[PS12] Jesse Peterson and Thomas Sinclair, On cocycle superrigidity for Gaussian actions, Ergodic Theory Dynam. Systems 32 (2012), no. 1, 249-272, DOI 10.1017/S0143385710000751. MR2873170

[Po83] Sorin Popa, Orthogonal pairs of *-subalgebras in finite von Neumann algebras, J. Operator Theory 9 (1983), no. 2, 253-268. MR703810 (84h:46077)

[Po86] S. Popa, Correspondences, INCREST Preprint 1986.

[Po93] Sorin Popa, Markov traces on universal Jones algebras and subfactors of finite index, Invent. Math. 111 (1993), no. 2, 375-405, DOI 10.1007/BF01231293. MR.1198815 (94c:46128)

[Po06a] Sorin Popa, On a class of type $\mathrm{II}_{1}$ factors with Betti numbers invariants, Ann. of Math. (2) 163 (2006), no. 3, 809-899, DOI 10.4007/annals.2006.163.809. MR.2215135 (2006k:46097)

[Po06b] Sorin Popa, Strong rigidity of $\mathrm{II}_{1}$ factors arising from malleable actions of w-rigid groups. I, Invent. Math. 165 (2006), no. 2, 369-408, DOI 10.1007/s00222-006-0501-4. MR2231961 (2007f:46058)

[Po07a] Sorin Popa, On Ozawa's property for free group factors, Int. Math. Res. Not. IMRN 11 (2007), Art. ID rnm036, 10, DOI 10.1093/imrn/rnm036. MR2344271 (2008m:46123)

[Po07b] Sorin Popa, Deformation and rigidity for group actions and von Neumann algebras, International Congress of Mathematicians. Vol. I, Eur. Math. Soc., Zürich, 2007, pp. 445477, DOI 10.4171/022-1/18. MR2334200(2008k:46186)

[Po08] Sorin Popa, On the superrigidity of malleable actions with spectral gap, J. Amer. Math. Soc. 21 (2008), no. 4, 981-1000, DOI 10.1090/S0894-0347-07-00578-4. MR2425177 (2009e:46056)

[PV10] Sorin Popa and Stefaan Vaes, Group measure space decomposition of $\mathrm{II}_{1}$ factors and $W^{*}$-superrigidity, Invent. Math. 182 (2010), no. 2, 371-417, DOI 10.1007/s00222-0100268-5. MR2729271 (2012b:46126)

[PV14a] Sorin Popa and Stefaan Vaes, Unique Cartan decomposition for $\mathrm{II}_{1}$ factors arising from arbitrary actions of free groups, Acta Math. 212 (2014), no. 1, 141-198, DOI 10.1007/s11511-014-0110-9. MR3179609

[PV14b] Sorin Popa and Stefaan Vaes, Unique Cartan decomposition for $\mathrm{II}_{1}$ factors arising from arbitrary actions of hyperbolic groups, J. Reine Angew. Math. 694 (2014), 215-239, DOI 10.1515/crelle-2012-0104. MR.3259044

[Sa89] J.-L. Sauvageot, Tangent bimodule and locality for dissipative operators on $C^{*}$-algebras, Quantum probability and applications, IV (Rome, 1987), Lecture Notes in Math., vol. 1396, Springer, Berlin, 1989, pp. 322-338, DOI 10.1007/BFb0083561. MR.1019584 (90k:46145)

[Sh00] Dimitri Shlyakhtenko, Free entropy with respect to a completely positive map, Amer. J. Math. 122 (2000), no. 1, 45-81. MR1737257 (2001f:46102)

[Sh09] Dimitri Shlyakhtenko, Lower estimates on microstates free entropy dimension, Anal. PDE 2 (2009), no. 2, 119-146, DOI 10.2140/apde.2009.2.119. MR2547131(2012a:46129) 
[Ue05] Yoshimichi Ueda, HNN extensions of von Neumann algebras, J. Funct. Anal. 225 (2005), no. 2, 383-426, DOI 10.1016/j.jfa.2005.01.004. MR2152505(2006k:46100)

[Va07] Stefaan Vaes, Rigidity results for Bernoulli actions and their von Neumann algebras (after Sorin Popa), Astérisque 311 (2007), Exp. No. 961, viii, 237-294. Séminaire Bourbaki. Vol. 2005/2006. MR2359046 (2009k:46112)

[Va08] Stefaan Vaes, Explicit computations of all finite index bimodules for a family of $\mathrm{II}_{1}$ factors (English, with English and French summaries), Ann. Sci. Éc. Norm. Supér. (4) 41 (2008), no. 5, 743-788. MR2504433 (2010k:46061)

[Va10] Stefaan Vaes, Rigidity for von Neumann algebras and their invariants, Proceedings of the International Congress of Mathematicians. Volume III, Hindustan Book Agency, New Delhi, 2010, pp. 1624-1650. MR2827858 (2012g:46006)

[Va12] Stefaan Vaes, An inner amenable group whose von Neumann algebra does not have property Gamma, Acta Math. 208 (2012), no. 2, 389-394, DOI 10.1007/s11511-0120079-1. MR2931384

[Vo93] Dan Voiculescu, The analogues of entropy and of Fisher's information measure in free probability theory. I, Comm. Math. Phys. 155 (1993), no. 1, 71-92. MR1228526 (94k:46137)

[Vo94] Dan Voiculescu, The analogues of entropy and of Fisher's information measure in free probability theory. II, Invent. Math. 118 (1994), no. 3, 411-440, DOI 10.1007/BF01231539. MR1296352 (96a:46117)

[Vo96] Dan Voiculescu, The analogues of entropy and of Fisher's information measure in free probability theory. III. The absence of Cartan subalgebras, Geom. Funct. Anal. 6 (1996), no. 1, 172-199, DOI 10.1007/BF02246772. MR.1371236 (96m:46119)

[Vo98a] Dan Voiculescu, A strengthened asymptotic freeness result for random matrices with applications to free entropy, Internat. Math. Res. Notices 1 (1998), 41-63, DOI 10.1155/S107379289800004X. MR1601878(2000d:46080)

[Vo98b] Dan Voiculescu, The analogues of entropy and of Fisher's information measure in free probability theory. V. Noncommutative Hilbert transforms, Invent. Math. 132 (1998), no. 1, 189-227, DOI 10.1007/s002220050222. MR1618636(99d:46087)

[Vo99] Dan Voiculescu, The analogues of entropy and of Fisher's information measure in free probability theory. VI. Liberation and mutual free information, Adv. Math. 146 (1999), no. 2, 101-166, DOI 10.1006/aima.1998.1819. MR.1711843 (2001a:46064)

[Vo02] Dan Voiculescu, Cyclomorphy, Int. Math. Res. Not. 6 (2002), 299-332, DOI 10.1155/S1073792802105046. MR.1877005 (2003a:46092)

[VDN92] Dan Voiculescu, K. J. Dykema, and A. Nica, Free random variables, A noncommutative probability approach to free products with applications to random matrices, operator algebras and harmonic analysis on free groups, CRM Monograph Series, vol. 1, American Mathematical Society, Providence, RI, 1992. MR.1217253 (94c:46133)

Université de Lyon, Université Lyon 1, Institut Camille Jordan UMR 5208, 43 BouleVARd Du 11 nOVEmbre 1918, F-69622 Villeurbanne Cedex, France

E-mail address: dabrowski@math.univ-lyon1.fr

Department of Mathematics, University of California, San Diego, California 900951555

E-mail address: aioana@ucsd.edu 